University of Louisville

ThinkIR: The University of Louisville's Institutional Repository

$5-2011$

\title{
Measurement of the electronic density of states of graphene oxide using capacitive photocurrent spectroscopy.
}

Tanesh Bansal

University of Louisville

Follow this and additional works at: https://ir.library.louisville.edu/etd

\section{Recommended Citation}

Bansal, Tanesh, "Measurement of the electronic density of states of graphene oxide using capacitive photocurrent spectroscopy." (2011). Electronic Theses and Dissertations. Paper 68.

https://doi.org/10.18297/etd/68

This Doctoral Dissertation is brought to you for free and open access by ThinkIR: The University of Louisville's Institutional Repository. It has been accepted for inclusion in Electronic Theses and Dissertations by an authorized administrator of ThinkIR: The University of Louisville's Institutional Repository. This title appears here courtesy of the author, who has retained all other copyrights. For more information, please contact thinkir@louisville.edu. 


\title{
MEASUREMENT OF THE ELECTRONIC DENSITY OF STATES OF GRAPHENE OXIDE USING CAPACITIVE PHOTOCURRENT SPECTROSCOPY
}

\author{
By \\ Tanesh Bansal \\ M.S. Electrical Engineering, University of Louisville \\ Louisville, Kentucky 2007

\begin{abstract}
A Dissertation
Submitted to the Faculty of the

Graduate School of the University of Louisville in Partial Fulfillments of the Requirements for the Degree of
\end{abstract}

\section{Doctor of Philosophy}

\author{
Department of Electrical \& Computer Engineering \\ University of Louisville \\ Louisville, KY 40292
}

May 2011 


\title{
MEASUREMENT OF THE ELECTRONIC DENSITY OF STATES OF GRAPHENE OXIDE USING CAPACITIVE PHOTOCURRENT SPECTROSCOPY
}

\author{
By \\ Tanesh Bansal \\ M.S. Electrical Engineering, University of Louisville, \\ Louisville, Kentucky 2007
}

A Dissertation approved on

April 23, 2011

By the following Dissertation Committee:

Dr. Bruce W. Alphenaar (Dissertation Director)

Dr. Robert W. Cohn

Dr. Cindy K. Harnett

Dr. Mahendra K. Sunkara

Dr. Gamini U. Sumanasekera 


\section{DEDICATION}

This dissertation is dedicated to my parents

Dr. Prakash Chand Bansal

$\&$

Mrs. Rita Bansal 


\section{ACKNOWLEDGMENTS}

I am deeply indebted to my advisor, Dr. Bruce Alphenaar, for giving me the opportunity to work under his immense guidance. I would like to express my gratitude for his support and guidance throughout the project. I am thankful for all the skills and techniques imparted to me under his mentorship. I would like to thank Dr. Gamini Sumanasekera for helping me with the Raman measurements of graphene and time to time discussions and suggestions. I would like to thank Dr. Mahendra Sunkara for authorizing the use of his lab facilities for the characterization measurements of devices. I also thank my committee members, Dr. Robert Cohn and Dr. Cindy Harnett for their advice and comments on this dissertation evaluation.

I would like to thank Prasanth Gopinath and Dr. Bhaskar Nagabhirava for their invaluable guidance in familiarizing me with the basic characterization instruments UVVIS-NIR absorption spectroscopy, atomic force microscopy, scanning electron microscope, e-beam lithography and other instruments. I would like to thank Mr. Mark Crain for familiarizing me with microfabrication processes and clean room routines during my early days as a researcher. I would like to recognize Dr. Aditya Mohite for familiarizing me with capacitive photocurrent spectroscopy. I also wish to thank Hemant Shah for helping me in figuring out the alignment and operation of OPA system. I wish to thank Dr. Jacek Jasinski for familiarizing me with the XPS measurements. I also wish to 
thank Kiran, Buddika and George for many interesting discussions on graphene, quantum dots, solar cells, spintronics and nanotechnology in general, and their inputs for fabrication process throughout the project. I thank all my fellow group members who have helped me in one way or the other and arranged a pleasant working environment in the lab throughout my stay at University of Louisville.

Finally, I would like to thank my family for their love, understanding, and support during the course of my doctoral studies. I would like to say special thanks to my brother for his constant support and encouragement. This work would not have been possible without their support. 


\begin{abstract}
MEASUREMENT OF THE ELECTRONIC DENSITY OF STATES OF GRAPHENE OXIDE USING CAPACITIVE PHOTOCURRENT SPECTROSCOPY
\end{abstract}

Tanesh Bansal

April $28^{\text {th }}, 2011$

Understanding of the band structure of the new nano materials is essential to have a deeper insight into their optical and electrical properties. Along with the existing techniques used for characterization of nano-materials, it is also important to develop new techniques and methods which can reveal previously unobserved details.

This dissertation describes an innovative measurement technique, capacitive photocurrent spectroscopy (CPS), which is used to probe the density of states (DOS) of graphene, graphene oxide (GO) and reduced graphene oxide (rGO). In contrast to the standard photocurrent measurement technique, CPS technique uses a floating electrode (electrodes separated by an insulator) configuration. Floating electrode eliminates the existence of any background current, which in turn increases the sensitivity towards photo generated electron/hole pairs revealing new features in the photocurrent spectrum. 
Also, this method eliminates the need for locating individual pieces on the substrate to make two contacts for current transport as required by the standard photocurrent method. The increase in sensitivity achieved by this technique can be exploited to gather absorption information from individual nano sized pieces, which is not possible with standard absorption spectroscopy.

Capacitive photocurrent spectroscopy measurements on graphene samples reveal a monotonic increase in the photocurrent spectrum with increasing incident photon energy (photon energy scanned from $0.5 \mathrm{eV}$ to $4 \mathrm{eV}$ ) together with an evidence of three reproducible peaks. The monotonous increase in the CPS spectrum is as expected from the semi-metallic graphene DOS. The presence of three peaks is attributed to unintentional oxidation of graphene samples. X-ray photoelectron spectroscopy (XPS) measurements of graphene samples confirm the presence of oxygen functional groups attached to the carbon atoms in graphene.

For GO, the monotonic background disappears, and the three peaks become the dominant features in the spectrum. Two of these peaks match well with published photoluminescence data, as transitions between the GO states and the $\pi / \pi^{*}$ graphene levels match the peak luminescence energy of GO. X-ray photoelectron spectroscopy measurements confirm that identical oxygen functional groups (as seen in graphene samples) exist (in varying quantities) for all the samples measured.

In the case of rGO samples, the background density of states due to the graphene reemerges along with additional states due to defects. The three peaks due to the GO also remain, indicating the continued presence of oxidized regions. X-ray photoelectron spectroscopy measurements confirm the presence of the identical oxygen functional 
groups, along with an extra feature indicating the presence of a carbon-nitrogen group (introduced during the reduction process).

Density of state calculations for graphene samples, based on the experimental results, indicate the presence of three small peaks near the vicinity of Fermi energy superimposed on the graphene DOS. For GO samples, DOS calculations revealed five peaks separated by hard gaps (no electron energy state), three of those are in the vicinity of Fermi energy (as seen in the case of graphene samples) while the remaining two resemble the $\pi / \pi^{*}$ peaks of graphene DOS. For rGO samples, many peaks with no hard gaps between them were revealed. The five peaks, three near Fermi energy and two resembling $\pi / \pi^{*}$ peaks (as seen in GO samples) are still present, along with more peaks.

In summary, a direct experimental probe of the GO density of states as a function of oxygen coverage using capacitive photocurrent spectroscopy is described. Three intense additional peaks in the DOS of GO appear near the Fermi energy (attributed to the presence of oxygen functional groups) along with standard $\pi / \pi^{*}$ peaks present in graphene DOS. Reduced graphene oxide ( $\mathrm{rGO}$ ) shows a distribution of peaks for the entire energy range probed in the experiment. Three similar peaks (less intense as compared to peaks seen in GO) with no hard gaps near Fermi energy were seen for the rGO sample providing the evidence of some residual oxidation. Extra peaks seen in rGO sample are attributed to defect creation during the reduction process. XPS measurements confirm the presence of similar oxygen functional groups in all the samples (Chemical Vapor Deposition, CVD grown graphene, GO and rGO) with varying intensity dictating the oxidation content percentage. 


\section{TABLE OF CONTENTS}

\section{PAGE}

DEDICATION................................................................................................................ iii

ACKNOWLEDGMENTS ................................................................................................ iv

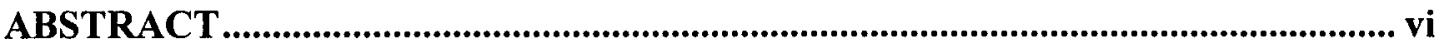

LIST OF FIGURES .............................................................................................. xii

\section{CHAPTERS}

I. INTRODUCTION .....................................................................................................1

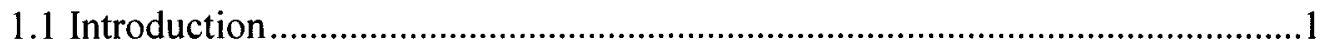

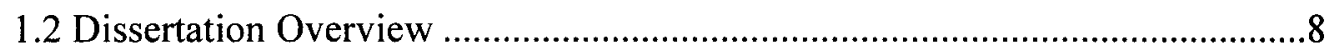

II. BACKGROUND ……...................................................................................10

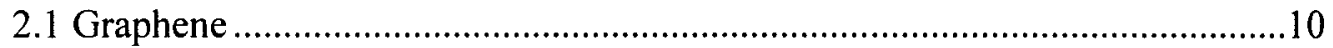

2.2 Carbon atom and Hybridization ......................................................................12

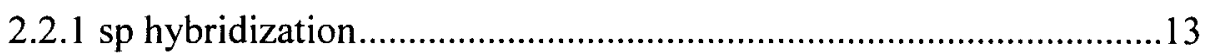

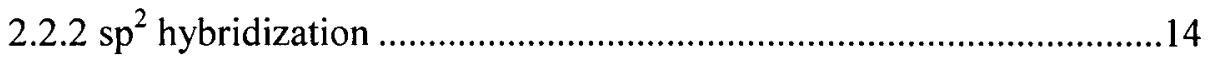

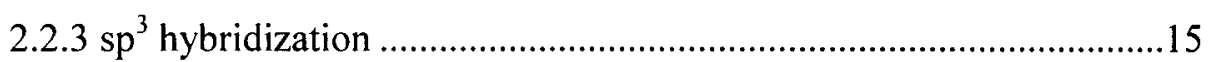


2.3 Band structure of the 5electronic states in graphene

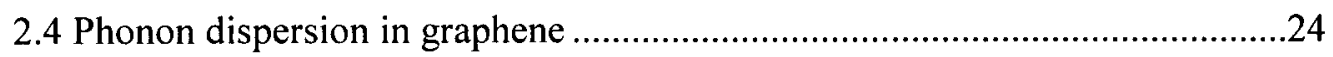

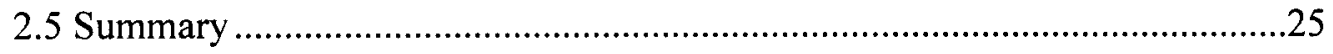

III. SYNTHESIS AND CHARACTERIZATION ...........................................................27

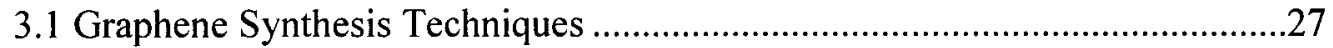

3.1.1 Mechanical exfoliation technique (scotch tape method) .................29

3.1.2 Chemically exfoliation technique (from GO to graphene) ..............31

3.1.3 Epitaxial growth on silicon carbide .............................................33

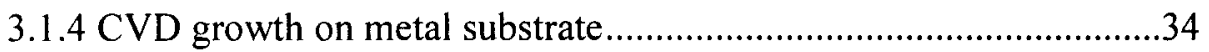

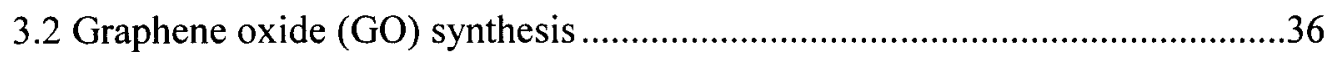

3.3 Reduced graphene oxide ( $\mathrm{GGO}$ ) synthesis techniques .................................38

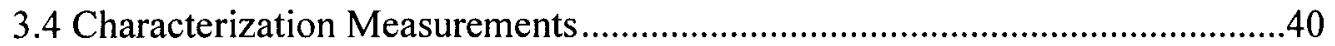

3.4.1 Electrical Measurements ........................................................40

3.4.2 Optical Measurements ..............................................................46

3.4.3 Raman processes in graphene …............................................50

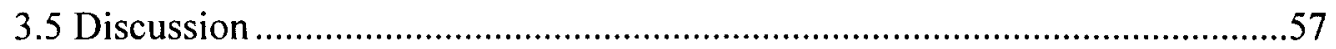

IV. PRELIMINARY MEASUREMETNTS AND CAPACITIVE PHOTO-

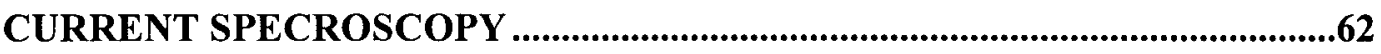

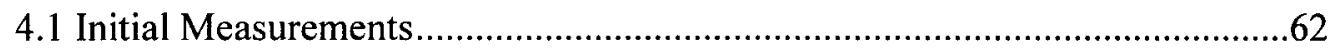

4.2 Capacitive Photocurrent Spectroscopy …...............................................66

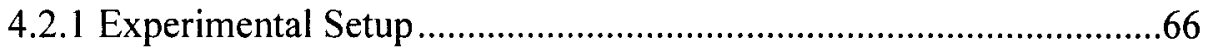

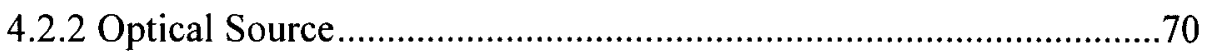


V. MEASUREMENT OF DOS OF GO AND rGO

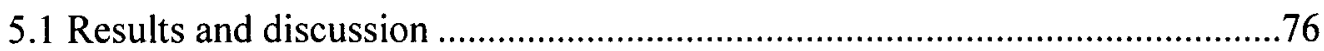

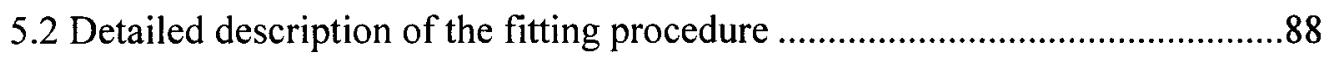

VI. CONCLUTIONS AND FUTURE DIRECTIONS...................................................94

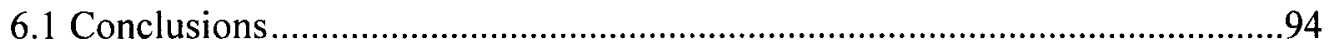

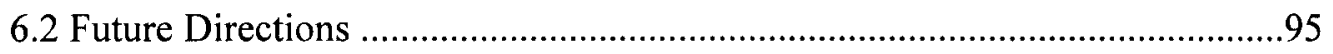

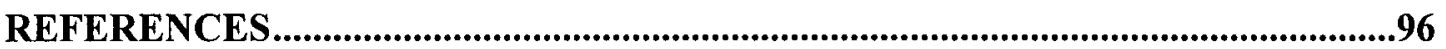

CURRICULUM VITAE....................................................................................................103 


\section{LIST OF FIGURES}

FIGURE

PAGE

2.1 Schematic of an idealized structure of a single graphene sheet. (adapted from reference [29]).

2.2 Schematic of bonding in graphene. a) in-plane $\sigma$-bonds. b) Out of plane pi bonds. The sideway overlapping of $2 p$ orbitals between neighboring carbon atoms form a pi bonding system that extends over all the carbon atoms, where a "cloud" of electrons can move about. (adapted from reference a [30], b [31]) .....................11

2.3 Schematic of sp hybridization. Two equivalent sp orbitals separated by $180^{\circ}$ form two strong $\sigma$ bonds, $\mathrm{p}_{\mathrm{y}}, \mathrm{p}_{\mathrm{z}}$ form two pi bonds resulting in $\mathrm{C} \equiv \mathrm{C}$ (triple bond between adjacent carbon atoms). (adapted from reference [36]).

2.4 Schematic of $\mathrm{sp}^{2}$ hybridization. a) Three equivalent $\mathrm{sp}$ orbitals. b) Planar orientation of the three sp orbitals. The three orbitals are separated by $120^{\circ}$ in $x-y$ palne having planar geometry and form three strong $\sigma$ bonds. (adapted from reference [38]).

2.5 Schematic of $\mathrm{sp}^{3}$ hybridization. a) Four equivalent $\mathrm{sp}$ orbitals, in three dimensions as a tetragonal structure, form four strong $\sigma$ bonds resulting in $\mathrm{C}-\mathrm{C}$ (single bond between adjacent carbon atoms). b) $\mathrm{sp}^{3}$ bonding in methane. (adapted from reference [39-40]).

2.6 (a) Graphene honeycomb structure showing the unit cell of two carbon atoms. (b) Real space lattice showing the periodic arrangements of the unit cells with basis vectors $\overrightarrow{a_{1}}$ and $\overrightarrow{a_{2}}$.

2.7 (a) Reciprocal lattice of graphene [42]. (b) Brillouin zone construction from the reciprocal lattice structure [42]. (c) Enlarged view of Brillouin zone, the coordinates for the six $\mathrm{K}$ points are also shown. Energy dispersion relations are obtained along the perimeter of the dotted triangle connecting the high symmetry points $\Gamma, \mathrm{K}$ and $\mathrm{M}$. .18 
2.8 (a) Schematic showing a unit cell $n$ connected to its neighbor unit cells $m$ by a matrix [41]. (b) Schematic showing a unit cell $n$, of graphene having two carbon atoms $\mathrm{A}$ and $\mathrm{B}$, connected to its four neighbor unit cells (where $m=1,2,3,4) \ldots \ldots .20$

2.9 (a) Energy dispersion of graphene. b) The dispersion of $\pi / \pi^{*}$ bands along the high symmetry directions $\Gamma$, M, K. c) Dispersion of all the $\sigma / \sigma^{*}$ and $\pi / \pi^{*}$ bands along $\Gamma$, $\mathrm{M}, \mathrm{K}$ high symmetry directions (adapted from $[39,43]$ ).

2.10 (a) Density of States (DOS) of. b) DOS shows linear behavior near corners where

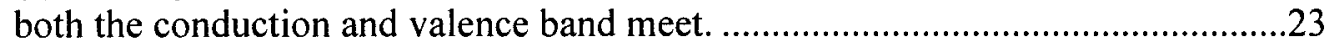

2.11 (a) Force constants between the $\mathrm{A}$ and $\mathrm{B} 1$ carbon atoms on a graphene sheet. Here, $\phi_{\mathrm{r}}, \phi_{\mathrm{ti}}, \phi_{\mathrm{to}}$ represents forces from the nearest neighbor atoms in the radial, bond stretching, (in-plane longitudinal modes, iLA, iLO), in-plane (iTA, iTO) and out of plane tangential (oTA, oTO) bond bending directions respectively [36]. (b) Calculated phonon dispersion curves plotted along high symmetry directions for a 2D graphene sheet. Phonon modes and their location along the high symmetry direction representing four features in the Raman spectra (D, G, $D^{\prime}$ and $\left.G^{\prime}\right)$ are also highlighted [47]. (c) Inelastic X-ray measurements and ab initio calculations of phonon dispersion curves in graphite (filled dots represent experimental data) [48].

3.1 Schematic of crystal structure of graphite showing ABAB stacking sequence and unit cell (adapted from [39]).

3.2 (A) Dr. Ruoff's approach of rubbing tiny pillars of graphite against Si. (a) HOPG sample, (b) Pillars formed on HOPG by oxygen plasma etching, (c) \& (d) examples of deposited graphene pieces. (B) Dr. Philip method of attaching graphite to AFM tip.(a) SEM image of AFM with graphite piece, (b) Schematic showing AFM tip with HOPG touching the substrate, (c) deposited graphene flakes, (d) device fabricated using one of the flakes. (C) Process of scotch tape deposition. (a) Graphite flakes, (b) Flakes were transferred to scotch tape using tweezers, (c) Pealing the sheets from the initial flake using second scotch tape, (d) Scotch tape with many thin graphene flakes. (D) Images of graphene pieces obtained by scotch tape method. (a) Optical image (lower magnification), (b) AFM image of graphene piece, (c) Optical image at higher magnification showing single graphene sheet.(adapted from $[33,51])$.

3.3 (A) (a) Schematic of intercalated material between the graphite layers, (b) Unexpanded intercalated graphite material, (c) \& (d) Expanded/ exfoliated graphite flakes. (B) Microwave assisted graphene exfoliation. (a) Exfoliated graphite. (b) SEM image of the dispersed graphene sample on the substrate. (adapted from A - [52], B - [55])

3.4 STM topographs of epitaxial graphene on $\mathrm{SiC}(0001)$ [56]. 34 
3.5 (a) Schematic of the CVD growth process flow. (b) $\mathrm{Cu}$ foil before growth. (c) $\mathrm{Cu}$ foil after growth. (d) PMMA/Graphene samples flowing on DI water. ..................36

3.6 (a) Schematic of a proposed structure of graphene oxide. (b) Graphene oxide film dispersed on $\mathrm{SiO}_{2} / \mathrm{Si}$ substrate (adapted from [59]) .........................................38

3.7 (a) TEM image of a partly folded rGO sheet. (b) Atomic resolution TEM image of single layer rGO sample with color added to highlight the different features(adapted from [63]). .39

3.8 (a) Ambipolar electric field effect in monolayer graphene at $1 \mathrm{~K}$. The inset shows the changes in the position of the Fermi energy $E_{\mathrm{F}}$ with changing gate voltage $\mathrm{Vg}$. (b) Dependence of few layer graphene's resistivity $\rho$, on gate voltage for different temperatures ( $\mathrm{T}=5,70$ and $300 \mathrm{~K}$ from top to bottom curves) (adapted from [8, 59]). 41

3.9 (a) Schematic of dual gate configuration with layer by layer description. (b) Transfer characteristics of dual gated bi-layer graphene device at room temperature. (c) Transfer characteristics of dual gated monolayer graphene device. (d) I-V characteristics of bi-layer graphene sample with back gate at $100 \mathrm{~V}$ and top gate being swept from -2 volts to 6 volts (adapted from [13])........42

3.10 (a) Schematic illustrating the bandstructure changes induced in bilayer graphene by an applied perpendicular electric field. The dotted curves represent the bandstructure in the absence of the field, while the solid line shows the bandstructure in the presence of a strong field. (b) Temperature dependence of bilayer graphene device (adapted from [22]).

3.11 (a)-(c) Conductance measurements of GNR devices as function of gate voltage measured at different temperatures. (d) Plot of energy gap versus width of GNR. (adapted from [14]) 43

3.12 Schematic process of making graphene nanoribbons suspensions (adapted from [16]). .44

3.13 (a) - (c) AFM images of GNR. (d) Plot of $\mathrm{I}_{\text {on }} / \mathrm{I}_{\mathrm{off}}$ ratio versus the GNR width. (e) Plot of energy gap versus the GNR width. (f) Gate dependence measurements for a $5 \mathrm{~nm}$ wide GNR. (g) I-V characteristics for the sample in (f). (adapted from [16]). .45

3.14 (a) I-V curve for different reduction steps. (b) Change in resistance with respect to gate voltage at different temperatures. Increasing temperature increase the reduction which in turn reducing the resistance and increasing the linearity of $\mathrm{I}-\mathrm{V}$ curve (adapted from [65]). 
3.15 (a) I-V curve for GO (before reduction). (b) $\mathrm{I}-\mathrm{V}$ curve for 4 different steps of reduction. (c) Change in resistance with respect to gate voltage at different temperatures (adapted from [66]).. 46

3.16 (a) I-V curve for GO (before reduction). (b) I-V curve for 4 different steps of reduction. (c) Change in resistance with respect to gate voltage at different temperatures (adapted from [23-27]).

3.17 (a)-(b) Adapted from Luo et al. [28], shows PL at $\sim 750 \mathrm{~nm}$. A small red shift in PL location with increasing reduction is shown in (b). (c)-(d) Adapted from Eda $t$ al. [25], PL at $\sim 390 \mathrm{~nm}$ is as shown. (c) Shows the change in PL intensity with increasing hydrazine reduction time. (d) Represents the change in PL intensity with reduction through annealing at different temperature. (e) Adapted from Sun et al. [27], shows GO PL at $\sim 570 \mathrm{~nm}$.

3.18 Schematic of the generalized Raman spectrum of graphene $([45]) \ldots \ldots \ldots \ldots \ldots \ldots \ldots . . . .50$

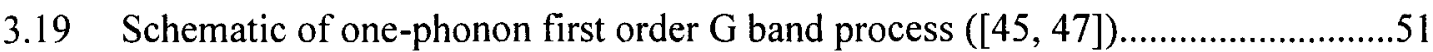

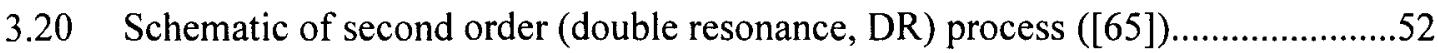

3.21 Schematic of second order (double resonance, DR) Raman process. D, D' and G'

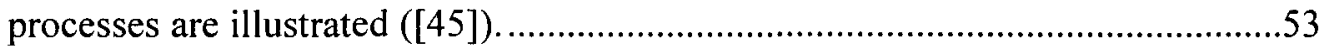

3.22 Schematic view of bi-layer graphene near $K, K^{\prime}$ points showing both $\pi_{1}$ and $\pi_{2}$ bands. The four DR processes are (a) $P_{11}$, (b) $P_{22}$, (c) $P_{12}$, (d) $P_{21}$. (e) G' band for bi-layer graphene with four fitted Lorentzians are also shown ([45]). .55

3.23 (a) Variation in Raman peaks shape and intensity with the change in the number of graphene layers. (b) Changes in Raman spectra of monolayer graphene with increasing fluorination. (c) Difference in Raman spectra for monolayer graphene, GO and rGO samples. (d) Evolution of Raman spectra of a monolayer graphene with hydrogenation and dehydrogenation $([24,65,68,69])$. .56

3.24 (a)-(d) ARPES measurements presenting the decrease in band gap with increase in number of layer grown. (e) Plot showing the energy band gap value versus number of layers ([12]). .58

3.25 (a)-(b) Adapted from Boukhlov et al., (a) three stable configuration of oxidized graphene with respective DOS. (b) Change in energy gap with change in oxygen coverage percentage. (c)-(d) Adapted from Lahaye et al., (c) DOS of partially oxidized graphene, with the majority (red) and minority (blue) spins separated. (d) DOS of fully oxidized graphene $([19,20])$

4.1 Optical image and XPS C1s curves for KISH graphite before and after 24 hours of acid treatment. 
4.2 Flow chart of the device fabrication procedure for electrical measurements. .......64

4.3 Optical image of graphene piece lying on the heavily doped $\mathrm{Si} / \mathrm{SiO}_{2}$ substrate. Patterned etch marks near the graphene piece shows a high resolution grid pattern used for locating the piece on the substrate for e-beam lithography. .64

4.4 Gate dependence measurements on graphene piece before and after acid treatment (oxidation) step. .65

4.5 Figure shows the basic setup for the capacitive photocurrent spectroscopy technique. The photocurrent is determined by measuring the output voltage using a lock-in amplifier set $90^{\circ}$ out of phase from the pulsed laser repetition frequency

4.6 Cross -section of ITO/Graphene heterojunction monitored by a cpacitively coupled probe electrode with (a) no light and (b) light on sample. .68

4.7 Optical transmission through a $30 \mathrm{~nm}$ film of ITO on quartz shows $>90 \%$ transmission between $0.5 \mathrm{eV}-3 \mathrm{eV}([70])$

4.8 Capacitive photocurrent response of a $\mathrm{p}$-Si plotted as a function of excitation photon energy shows the typical band edge of silicon at $\sim 1.1 \mathrm{eV}([70])$. .70

4.9 A digital camera image of the OPA aligned next to a Hurricane which pumps the OPA .71

4.10 The four subsystems of Hurricane: Mai Tai, Evolution, stretcher/compressor and regenerative amplifier ([72]) .72

5.1 X-ray photoelectron spectra (red curves) of GO, rGO and CVD grown graphene samples with de-convoluted components (light colored curves). . .77

5.2 Schematic of the experimental setup (left) and the equivalent band diagram (right) for the CPS measurement.

5.3 Capacitive photocurrent spectra as a function of excitation energy for four different individual graphene sheets. (a) \& (b) Graphene transferred using $10 \%$ aqueous solution of $\mathrm{HNO}_{3}$. (c) \& (d) Graphene transferred using ferric chloride solution. Samples ((a) and (c)) were measured using the standard measurement configuration shown in Figure 1(a), while the other two (b) and (d) were measured with the graphene lying on quartz, with a remote silver paste contact placed on a corner of the graphene sheet. Reproducible peaks near $0.7 \mathrm{eV}, 1.6 \mathrm{eV}$ and $3.2 \mathrm{eV}$ are marked with $\boldsymbol{\Delta}, \bullet$, and $\boldsymbol{m}$, respectively. The dashed lines show the calculated spectrum following equation (1) and using density of states, $D(E)$, of graphene as shown in (e). 
5.4 Capacitive photocurrent spectra of four GO samples (solid blue line). Three peaks are observed at $0.7,1.6$ and $3.2 \mathrm{eV}$, which correlate with the peak positions observed in the graphene (marked as $\boldsymbol{\Lambda}, \bullet$, and $\mathbf{m}$, respectively). The dashed red curves are calculated following equation (1) and using the density of states for GO as shown in Figure 5.5.

5.5 Predicted density of states for GO following the fitting of equation (2) to the data plotted in Figure 5.4. Transitions between the filled and empty states (as shown by the $\boldsymbol{\Delta}, \bullet$, and $\boldsymbol{\square}$ transitions in (a)) produce the peaks observed in the capacitive photocurrent.

5.6 Experimental results of capacitive photocurrent spectroscopy on rGO (solid blue curves). Three peaks, on top of a monotonically increasing background, are observed at $0.7,1.6$ and $3.2 \mathrm{eV}$, (marked as $\boldsymbol{\Delta}$, $\bullet$, and $\mathbf{\mathbf { m }}$, respectively). The dotted curve is calculated following equation (1) and using the density of states for rGO as shown in Figure 5.7.

5.7 Predicted density of states for rGO following the fitting of equation (2) to the data plotted in Figure 5.6. Transitions between the filled and empty states (as shown by the $\boldsymbol{\Delta}, \bullet$, and $\boldsymbol{\|}$ transitions in (a)) produce the peaks observed in the capacitive photocurrent. .86

5.8 Diagram showing the discrete DOS, $D_{ \pm j}$, and the measured capacitance photocurrent $C_{i}$. Points marked with an $\mathrm{x}$ are unmeasured $\mathrm{C}_{\mathrm{i}}$ values below $\mathrm{C}_{\mathrm{im}} \ldots \ldots \ldots \ldots . . .88$

5.9 CPS of first device of GO, dots represent the interpolated points used for fitting procedure.

5.10 Calculated capacitive photocurrent for a constant DOS .90

5.11 Demonstration of fitting procedure after number of runs $(n=1,100,10000) \ldots \ldots . .91$

5.12 Three calculated DOS curves for one GO device. (d) Shows the Lorentzian fit to the peaks seen in calculated DOS.

5.13 Fitting procedure used for the DOS curves shown in Figure 5.12 .93 


\section{CHAPTER I INTRODUCTION}

\subsection{Introduction}

Over the last several decades, semiconductor technology has developed considerably. The driving force has been the miniaturization of the channel length of the device, which leads to devices being much more advanced in their performance. Specifically they are much faster, lighter and more robust. They dissipate less heat, consume less power and have a much lower cost $[1,2]$. Enhanced device performance through reduction in active feature size has worked so far. However, the current technology is at a point where within a decade the physics of the device operation will be acting as a roadblock for further shrinking of the feature size. As the device sizes are reduced, denser device arrays are required which in turn make the current material of choice (silicon) obsolete. At a very small size scale silicon generates too much resistance to electron flow resulting in the creation of more heat than can be dissipated and it consumes much more power to drive the circuit [3]. Also, high circuit density requires thinner metal interconnects carrying high density of current at higher speed. However, at small size scales, electro-migration limits the usefulness of standard metals for current flow, which is pushing researches to look for new materials and techniques for interconnects [4]. 
Two basic requirements govern the switching speed and power consumption of the switching circuitry. Faster charge transport within the device (source to drain) and faster charge transport between different active components of the circuit (interconnects) makes the first requirement, while having superior control between the on and off state of the device works as the second requirement. The leakage current in the off state influences the overall power consumption and heat dissipation of the device. The current trend of reducing the channel length of the device has done the trick until now. The decrease in the channel length reduces the overall distance to be covered by the charges which in turn produces faster devices. However, this increase in performance is still limited by the intrinsic properties, such as carrier mobility and energy band gap, of the material (silicon) used as well as the transport mechanism (diffusion) involved. Also, the reduction in feature size is reaching its limit. As the channel length is becoming comparable to the electron mean free path (tens of $\mathrm{nm}$ ) various new phenomena involving quantum mechanical effect become important [5-7]. They include conductance quantization due to ballistic electron motion, conductance fluctuations due to quantum interference effects, and Coulomb blockade or single-electron tunneling due to the discreteness of the electronic charge. At this length scale, the transport mechanism changes from being diffusive to ballistic and the influence of the contact resistance between source/drain with metal interconnects start dominating the overall device resistance.

The emergence of these new physical mechanisms (quantum effects) in the device operation has spurred the semiconductor industry and researchers to look for new materials and techniques to exploit these quantum phenomena for device applications. In the process of looking for new material for nano-electronics applications, graphene 
has emerged as a prospective candidate for replacing or being integrated into Si-based electronics. Graphene is the name given to a single layer of graphite [8]. It is the thinnest known material, only one carbon atom thick, and the strongest material measured util now. Several features make graphene's electronic properties unique and different from those of any other known condensed matter system and emphasize graphene's long-term prospects in becoming the base electronic material for computer electronics. It exhibits very high carrier mobility $\sim 200000 \mathrm{~cm}^{2} \mathrm{~V}^{-1} \mathrm{~s}^{-1}$ for suspended samples while for substrate supported $\left(\mathrm{SiO}_{2}\right)$ samples the reported mobility fluctuates from few thousands to tens of thousands $\mathrm{cm}^{2} \mathrm{~V}^{-1} \mathrm{~s}^{-1}$, depending upon the nature and purity of the insulator layer, as compared to maximum electron mobility of silicon $\left(1400 \mathrm{~cm}^{2} \mathrm{~V}^{-1} \mathrm{~s}^{-1}\right)$ [9]. Graphene shows very low electron scattering at room temperature, and exhibits ballistic transport at room temperature for channel lengths in the range of couple of hundreds of nanometer $(\sim 200 \mathrm{~nm})$ [9]. Graphene can sustain current densities $\left(\sim 10^{8}-10^{9} \mathrm{~A} \mathrm{~cm}^{-2}\right)$ many orders of magnitude higher than that of copper $\left(\sim 10^{4}-10^{5} \mathrm{~A} \mathrm{~cm}^{-2}\right)[4]$ and displays high thermal conductivity $\left(3000-5000 \mathrm{~W} \mathrm{~m}^{-1} \mathrm{~K}^{-1}\right)[10]$.

Exploiting the high carrier mobility of graphene, faster devices can be envisioned even without reducing the channel lengths. Also, the high carrier mobility of graphene has the potential for increasing the frequency of the microprocessor to the order of $100 \mathrm{GHz}$ [11]. Ambipolar nature of graphene enables the possibility of changing the device characteristics from p-type to n-type and vice versa just by modulating the gate voltage [8]. High current density combined with its true two-dimensional (2D) structure makes it an ideal candidate for nano-scale interconnects. The high current carrying capacity makes it very robust in resisting electromigration which in turn should greatly 
improve chip reliability while the true 2D nature greatly reduces the capacitive coupling between adjacent wires [4]. The high thermal conductivity of graphene will allow graphene interconnects to also serve as heat spreaders which will reduce device failures due to heat damage.

Graphene in many ways is an ideal electronic material. However, it is a zero-band gap semiconductor at low temperatures and behaves as semimetal at room temperature. Being a zero band gap material, graphene conductance does not change appreciably with gate voltage. The zero band gap also limits graphene from showing any appreciable photocurrent or absorption which reduces the possibility for optical applications. Graphene-based integrated circuits would require the conducting channel to be completely closed in the off state. Graphene's gapless electronic spectrum must be modified to have an energy band gap for feasible electronic applications. The challenge now facing graphene electronics and optoelectronics research is to develop new methods for introducing the energy band gap into graphene without significantly destroying the high carrier mobility exhibited by the pristine graphene.

Various approaches have been developed for opening an energy band gap between the valence and the conduction band of graphene and improving its semiconducting behavior. Researchers have shown that, it is possible to open up a small band gap (approximately $0.3 \mathrm{eV}$ ) through the interaction of graphene with the substrate, or through inter-graphene layer interactions; however the gap size is difficult to control, and is too small for most device applications [12]. Applying a perpendicular electric field to a bilayer graphene sheet opens up a small energy gap (approximately $0.3-0.5 \mathrm{eV}$ ) between the valence and the conduction band [13]. However, the band gap is difficult to control 
and too small for most device applications, applying a perpendicular electric field requires dual gate (top gate and bottom gate) configuration which further increases the fabrication complexity. Another approach for band gap opening utilizes the 2D nature of graphene. If one of the dimensions (width) of the graphene sheet is reduced to the order of a couple of nanometers, $(2 \mathrm{~nm}-25 \mathrm{~nm})$ the quantum confinement effect takes control and results in the formation of an energy band gap [14-16]. The magnitude of the band gap is hard to control as it critically depends on the width of the graphene nano-ribbon (GNR) as well as the smoothness and structure (armchair or zig-zag) of the atomic-scale edge of the GNR. As seen in the semiconductor industry, electronic properties of a material can be modified following a doping event. Following this idea, graphene oxidation has been pursued with great interest. Attempts to oxidize graphene following the methods used for graphite oxidation result in changing the conduction properties of graphene from semimetal to insulator, suggesting a large band gap opening $[17,18]$.

Among the various approaches pursued for an energy band gap opening in graphene, oxidation of graphene has emerged as a potential candidate for research. Recent theory calculations emphasis the idea that oxidation provides a possible method to create a tunable band-gap in graphene $[19,20]$. Theory further proposes that there are number of stable oxidative states of graphene oxide (GO) that produce characteristic electronic states for specific oxygen coverage. In other words, the oxygen/carbon ratio directs the number of states at each energy level that are available to be occupied by electrons (density of states, DOS) in GO. The experimental study shows that fully oxidized graphene is insulating at room temperature, but can be made conducting by partial reduction of the oxygen using hydrazine. These results have been interpreted as being due 
to the decrease in the band-gap of oxidized graphene with decreasing oxygen/carbon ratio. If correct, this could allow for the development of graphene optoelectronic devices, including tunable graphene photo-detectors and light emitting diodes. However, conductance measurements on their own do not provide very strong evidence for an optically accessible band gap, because scattering from physisorbed and chemisorbed oxygen atoms, or oxygen doping (particularly in the presence of a substrate induced gap) can also influence the graphene conductance [21]. Also, conductance measurements can be influenced by the metal contacts attached to $\mathrm{GO}$ and reduced graphene oxide (rGO) for injection and collection of charge carries. These metal contacts may introduce potential energy barrier and local doping effect at the interface [22]. Therefore, without a direct measure of the optical band-gap, it is difficult to distinguish between these different scenarios.

The distribution of electronic states or density of states (DOS) near the Fermi energy governs the electrical and optical properties of any material. Measuring the DOS of GO and $\mathrm{rGO}$ will provide a better insight on the relationship between the oxygen coverage with the size of the energy band gap. However, the methods used for measuring the DOS of graphene such as Scanning Tunneling Spectroscopy (STS) and Angle Resolved Photoemission Spectroscopy (ARPS) are more difficult to use for GO due to its insulating nature. The main methods for exploring the GO DOS have optical absorbance and photoluminescence spectroscopy. Graphene itself is nearly transparent in visible and infra-red region and shows a broad absorbance spectrum in the ultraviolet (UV) region, peaked at approximately $260 \mathrm{~nm}[23,24]$. Similarly, GO shows a broad absorption spectra in UV region, peaked at $\sim 233 \mathrm{~nm}$ which is attributed to the $\pi \rightarrow \pi^{*}$ of $\mathrm{C}=\mathrm{C}$ and a 
small shoulder at $\sim 320 \mathrm{~nm}$, corresponding to $n \rightarrow \pi^{*}$ transition of the $\mathrm{C}=\mathrm{O}$ bond $[25,26$, 27]. The similarity in the broad absorption feature of graphene and GO indicates that graphene DOS still influences the GO DOS. The photoluminescence measurements on GO samples shows a broad emission peak, centered at $570 \mathrm{~nm}(2.18 \mathrm{eV})$ by one group (Sun et al.) [27], at $390 \mathrm{~nm}(3.2 \mathrm{eV})$ by another group (Eda et al.) [25], and at $750 \mathrm{~nm}$ $(1.65 \mathrm{eV})$ by a third group (Luo et al.) [28]. The lowest energy (1.65 eV, 750nm) luminescence is from a GO sample while the highest energy $(3.2 \mathrm{eV}, 390 \mathrm{~nm})$ is due to moderately reduced graphene oxide sample and the medium energy $(2.18 \mathrm{eV}, 570 \mathrm{~nm})$ is due to GO nano-strips. The peak luminescence is clearly dependent on the synthesis conditions, however, no strong dependence on oxygen concentration has been observed. While it is clear that the luminescence is due to transitions among states introduced between the $\pi \rightarrow \pi^{*}$ peaks, the location of the peaks and their dependence on the oxygen concentration is unknown.

Very high conductivity and high optical transmission of graphene inhibits the use of standard two terminal photocurrent spectroscopy routinely used for measuring the density of states of semiconductors. The background current surpasses the small change produced by the optical transitions in the graphene samples. On the other hand, GO is an insulator which again makes this measurement impractical due to the absence of any current flow.

Probing of DOS of GO by optical spectroscopy techniques (absorption and photoluminescence) provides partial information regarding the distribution of states near the Fermi energy. However, more comprehensive picture of the DOS is required to confirm the influence of oxygen concentration on the opening of band gap as predicted 
theoretically. Addressing this problem of measuring the DOS experimentally is the premise of this dissertation study.

Importance of the proper knowledge of DOS of GO with varying oxygen/carbon ratio and the fact that traditional measurement techniques have shown limited promise has motivated this research study. In this research study, DOS of several samples of graphene, GO and rGO (synthesized under varied conditions) were measured using a novel Capacitive Photocurrent Spectroscopy (CPS) technique.

The main thrust to this dissertation is to determine the density of states of graphene oxide based on experimental evidence. Also, the perturbation in the energy states location and their density in the DOS of GO with respect to the changes in the oxygen concentration will be examined.

\subsection{Dissertation Overview}

As the title of the dissertation suggests, this dissertation mainly revolves around presenting a novel technique (CPS) to measure the electronic state distribution for graphene, GO and rGO samples. Several variations in the synthesis procedures of graphene, GO and rGO have been exploited to observe the effects of oxygen concentration changes on the DOS measurements. Various characterization measurements such as electrical conductivity measurements, Raman spectroscopy and Xray photoelectron spectroscopy (XPS) are performed to record the variation spread among dissimilar samples. Electrical conductivity measurements mainly involve the gate voltage dependence of devices which will help in sorting the devices as semimetal, 
semiconductor or as an insulator. Raman spectroscopy is used to check the defect levels in the graphene, GO and rGO samples. XPS measurement provided the list of oxygen functional groups attached to all the three types of samples.

The dissertation is organized as follows. Chapter II provides a basic review of graphene explaining its structure, types of chemical bonding involved. The first part introduces carbon hybridization in general and its effects on band structure of graphene. Calculations for energy dispersion relation of graphene, its band structure and its DOS are presented in the later part of the chapter. Phonon dispersion in graphene concludes the discussion in the chapter.

Chapter III provides the general synthesis and characterization techniques for all the three types of samples: graphene, GO and rGO.

Chapter IV provides the experimental setup and observations for the initial investigations. The second part of the chapter provides a brief description of the capacitive photocurrent measurement and the experimental setup involved.

Chapter $\mathrm{V}$ provides the experimental setup and results for the main research work. The second part of the chapter presents the DOS results based on the CPS measurements.

Chapter VI presents the conclusions drawn from the dissertation study and some possible directions for further work. 


\section{CHAPTER II}

\section{BACKGROUND}

\subsection{Graphene}

Graphene is the name given to a single layer of graphite. Graphene consists of a single layer of carbon atoms arranged in a two dimensional (2D) hexagonal honeycomb pattern as shown Figure 2.1 [29], thus making it the thinnest known material.

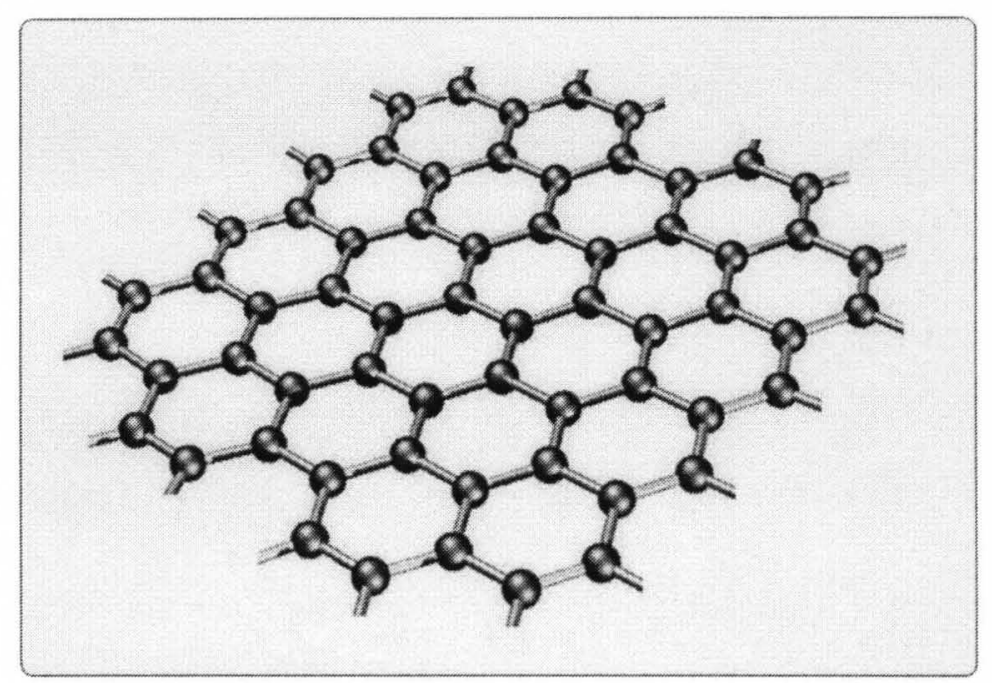

Figure 2.1 Schematic of an idealized structure of a single graphene sheet. (adapted from reference [29]).

The atomic structure of graphene is characterized by $\mathrm{sp}^{2}$ hybridization of carbon atoms resulting in two types of covalent bonds, sigma $(\sigma)$ and pi $(\pi)$, constructed from the combination of four valence orbitals $\left(2 s, 2 p_{x}, 2 p_{y}, 2 p_{z}\right)$. 
The three in-plane sigma $(\sigma)$ bonds join a carbon atom to its three nearest neighbors while the fourth bond is formed due to the sideways overlap of the $p_{z}(\pi)$ orbitals as shown in Figure $2.2[30,31]$. The in-plane $\sigma$ bonds and the side overlap of $\pi$ bonds strongly connect the carbon atoms forming a carbon hexagonal network and are responsible for the large binding energy and the elastic properties of the graphene sheets.

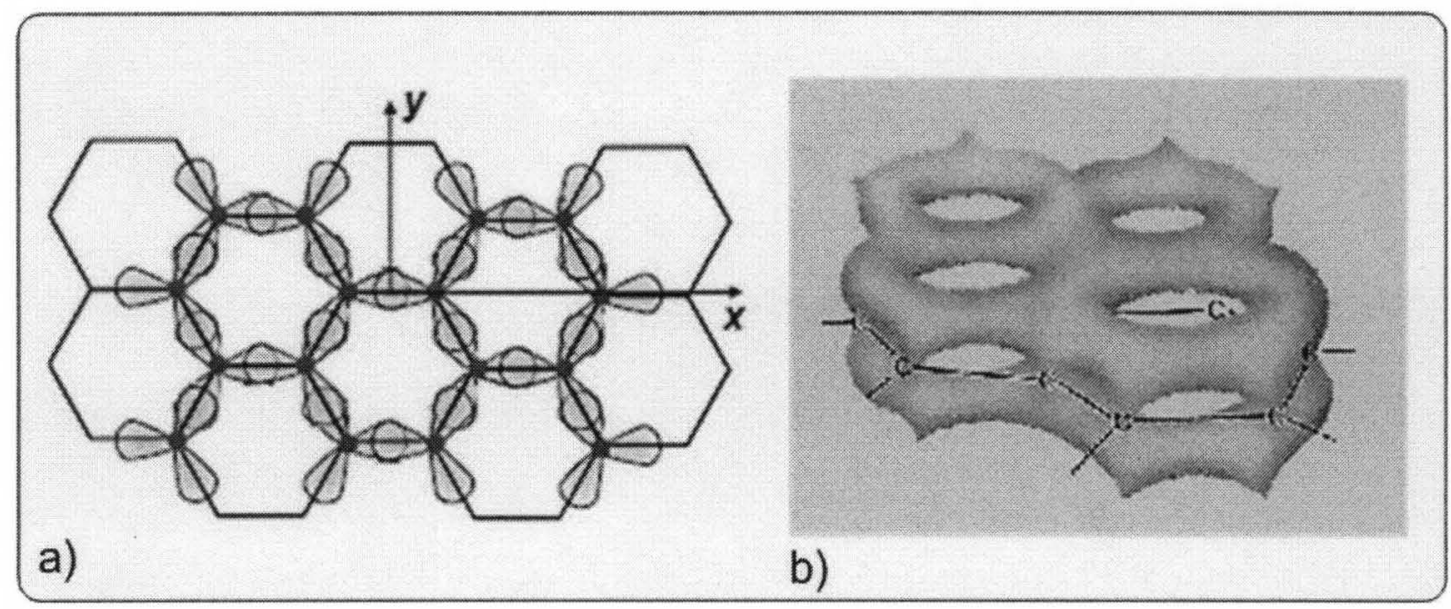

Figure 2.2 Schematic of bonding in graphene. a) in-plane $\sigma$-bonds. b) Out of plane pi bonds. The sideway overlapping of $2 \mathrm{p}$ orbitals between neighboring carbon atoms form a pi bonding system that extends over all the carbon atoms, where a "cloud" of electrons can move about. (adapted from reference a [30], b [31]).

The bonding $\sigma$ and antibonding $\sigma^{*}$ orbitals are separated by large energy gap of $\sim 12 \mathrm{eV}$, while the bonding $\pi$ and antibonding $\pi^{*}$ lie in the vicinity of the Fermi energy $\left(\mathrm{E}_{\mathrm{F}}\right)$ and direct the electrical and optical properties of the graphene [32]. The $\pi$ band electronic dispersion for graphene near the six corners of the 2D hexagonal Brillouin zone is found to be linear which results in formation of cones of carriers (electrons and holes) appearing at the six corners of the $2 \mathrm{D}$ Brillouin zone. At the Fermi energy these cones touch at six points called Dirac points which reduced the Fermi surface to the six points at the corners of the $2 \mathrm{D}$ hexagonal Brillouin zone and turns graphene into a zeroband gap semimetal [33]. 
Graphene shows exceptional electrical and mechanical properties. In short, very high carrier mobilities, low electron scattering, ballistic transport behavior at room temperature existing up to a couple of hundred of nanometers, high current carrying capacity, and high thermal conductance $[3-4,8-10,32-34]$. As far as mechanical properties are concerned, a suspended single layer of graphene is one of the stiffest known materials characterized by a remarkably high Young's modulus of $\sim 1 \mathrm{TPa}$ and intrinsic strength of $130 \mathrm{GP}$ [35].

In summary, graphene's remarkable electronic, optical and mechanical properties are the direct consequence of the crystal structure and $\mathrm{C}-\mathrm{C}$ bonding of graphene. The next section details the concept of carbon hybridization which will provide better understanding of the bonding in graphene.

\subsection{Carbon atom and Hybridization}

Carbon is the sixth element in the periodic table having six electrons occupying $1 \mathrm{~s}^{2}$, $2 \mathrm{~s}^{2}, 2 \mathrm{p}^{2}$ atomic orbitals. The strongly bound $1 \mathrm{~s}^{2}$ electrons are called core electrons and are not available for bonding, while the four $2 \mathrm{~s}^{2}, 2 \mathrm{p}^{2}$ electrons are loosely bound and act as valence electrons for further bonding with other atoms. Compared to the binding energy of chemical bonds, the energy difference between $2 s^{2}$ and $2 p^{2}$ is very small; one electron from $2 s$ energy level can jump to $2 p$ energy level resulting in highly active $2 s^{1}$, $2 \mathrm{p}^{3}$ state. There are then four unpaired electrons, whose wave functions are the result of a hybridization of the four orbitals, $2 \mathrm{~s}, 2 \mathrm{p}_{\mathrm{x}}, 2 \mathrm{p}_{\mathrm{y}}$ and $2 \mathrm{p}_{\mathrm{z}}$. In other words, the electronic wave functions for these four electrons can mix readily with each other resulting in new 
orbitals. This inter-mixing of electron wave functions to form new orbitals is called hybridization which enhances the binding energy of each carbon atom with its neighboring atoms [36-40]. Depending on the number of $2 \mathrm{p}$ orbitals involved in hybridization with the $2 \mathrm{~s}$ orbital different hybridization configurations are possible (sp, $\left.\mathrm{sp}^{2}, \mathrm{sp}^{3}\right)$. The various hybridization types are as follows.

\subsection{1 sp hybridization:}

This involves combination of the $2 \mathrm{~s}$ orbital and one of the $2 \mathrm{p}$ orbital's $\left(2 \mathrm{p}_{\mathrm{x}}, 2 \mathrm{p}_{\mathrm{y}}\right.$ or $\left.2 p_{z}\right)$. For example, $2 \mathrm{~s}$ hybridizes with $2 \mathrm{p}_{\mathrm{x}}$ to form two new hybridized orbitals $\left(\mathrm{sp}_{\mathrm{a}}, \mathrm{sp}_{\mathrm{b}}\right)$, resulting in two equivalent bonding sites along the $\mathrm{x}$-axis. The notable property of hybridized orbitals is that they are larger in amplitude in one direction and smaller in the other. The $\mathrm{sp}_{\mathrm{a}}$ hybridized orbital is stronger or elongated in the positive $\mathrm{x}$-direction while smaller or shrunk in the negative $\mathrm{x}$-direction. Similarly, for the $\mathrm{sp}_{\mathrm{b}}$ hybridized orbital, the amplitude is more in the negative $\mathrm{x}$-direction as compared to the amplitude in positive $\mathrm{x}$ direction (as shown in Figure $2.3[36]$ ).

Thus, when nearest-neighbor atoms are in the positive $\mathrm{x}$-direction, the overlap between $\mathrm{sp}_{\mathrm{a}}$ orbital and nearest neighbor orbital becomes large compared with the original $2 \mathrm{p}_{\mathrm{x}}$ orbital overlap, resulting in lower total energy (or higher binding energy). The bond formation due to the overlap of $\mathrm{sp}$ orbital with the nearest neighbor atomic orbital is also called as the sigma $(\sigma)$ bond. Similarly, nearest-neighbor in negative $\mathrm{x}-$ direction will form sigma $(\sigma)$ bond using $\mathrm{sp}_{\mathrm{b}}$ hybridized orbital. The remaining two electrons are in $2 p_{y}$ and $2 p_{z}$ orbitals, that is in perpendicular direction with respect to the 
hybrid orbitals, and they interact (sideways overlapping) with p-orbitals of the nearest neighbor and form weaker bond also known as the pi $(\pi)$ bond.

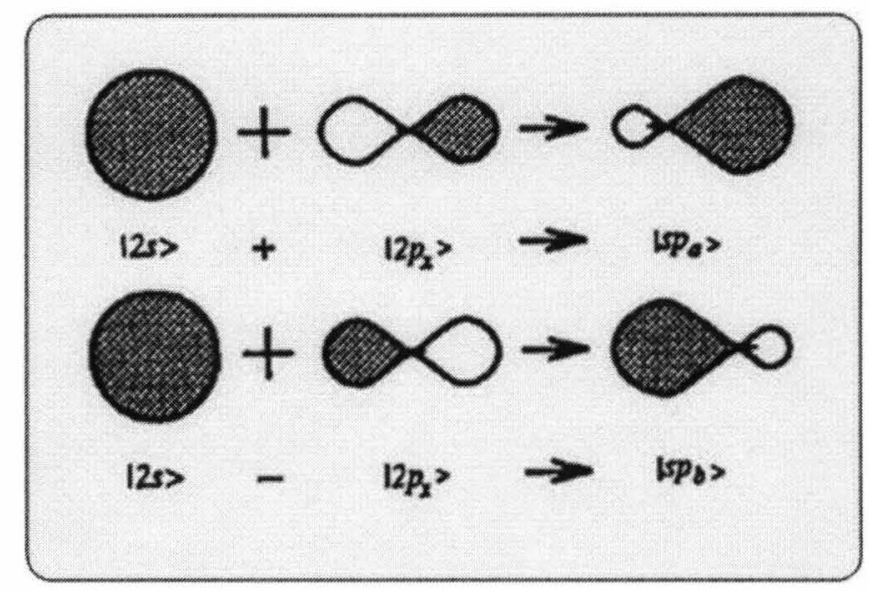

Figure 2.3 Schematic of sp hybridization. Two equivalent sp orbitals separated by $180^{\circ}$ form two strong $\sigma$ bonds, $\mathrm{p}_{\mathrm{y}}, \mathrm{p}_{\mathrm{z}}$ form two pi bonds resulting in $\mathrm{C} \equiv \mathrm{C}$ (triple bond between adjacent carbon atoms). (adapted from reference [36]).

Larger overlap between the bonding orbitals results in stronger bonds (sigma, $\sigma$ ) while less overlap results in relatively weaker bonds $(\mathrm{pi}, \pi)$.

\subsection{2 $\mathrm{sp}^{2}$ hybridization:}

This involves combination of the $2 \mathrm{~s}$ orbital and two of the $2 \mathrm{p}$ orbital's $\left(2 \mathrm{p}_{\mathrm{x}}, 2 \mathrm{p}_{\mathrm{y}}\right.$ or $2 \mathrm{p}_{\mathrm{z}}$ ). For example, $2 \mathrm{~s}$ hybridizes with $2 \mathrm{p}_{\mathrm{x}}$ and $2 \mathrm{p}_{\mathrm{y}}$ to form three equivalent in-plane bonding sites, aligned at $120^{\circ}$ with each other. This configuration results in three strong sigma $(\sigma)$ bonds and one weak pi $(\pi)$ bond. The sigma bonds ( $\sigma$ bonds) are in the xy planes while the $\pi$ bond is perpendicular to the plane. The $\mathrm{sp}^{2}$ orbitals thus results in a trigonal bonding arrangement having large amplitude in the three directions $\left(120^{\circ}\right.$ apart $)$ within the xy plane as shown in Figure 2.4 [38]. A common carbon-based example of $\mathrm{sp}^{2}$ 
hybridization is polyacetylene $(\mathrm{HC}=\mathrm{CH}-)_{\mathrm{n}}$. Graphene is another example of $\mathrm{sp}^{2}$ hybridization having only carbon-carbon bonds (three $\sigma$ bonds and one $\pi$ bond).

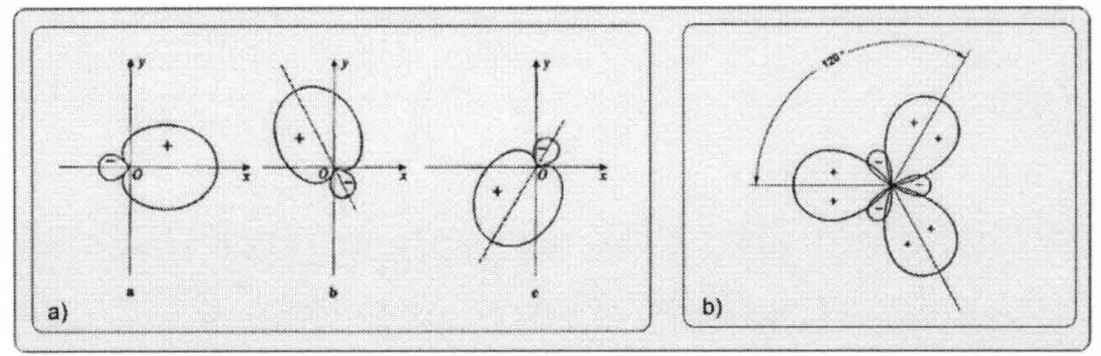

Figure 2.4 Schematic of $\mathrm{sp}^{2}$ hybridization. a) Three equivalent sp orbitals. b) Planar orientation of the three sp orbitals. The three orbitals are separated by $120^{\circ}$ in $x-y$ palne having planar geometry and form three strong $\sigma$ bonds. (adapted from reference [38]).

As previously mentioned, $\mathrm{sp}^{2}$ hybridization governs the electrical and mechanical properties of graphene. Graphene planar geometry is the direct consequence of $\mathrm{sp}^{2}$ hybrid sigma $(\sigma)$ bonds resulting in hexagonal pattern in the $x-y$ plane. The sideways overlapping of the pi orbitals of the neighboring carbon atoms results in a pi bonding system which forms a pi molecular orbital that extends over all the carbon atoms of the graphene sheet. The delocalization of electrons within this pi bonding structure allows the electrons to move around, forming a cloud of $\pi$ electrons located above and below the molecular plane, ensuing increased conductivity. This delocalization of electrons helps in reducing the overall energy of the system making it more stable, which in turn reduces chemical reactivity.

\subsection{3 $\mathrm{sp}^{3}$ hybridization:}

In $\mathrm{sp}^{3}$ hybridization, the $2 \mathrm{~s}$ orbital overlaps with all the three $2 \mathrm{p}$ orbitals, $2 \mathrm{p}_{\mathrm{x}}, 2 \mathrm{p}_{\mathrm{y}}$ and $2 \mathrm{p}_{\mathrm{z}}$ creating four equivalent bonding sites at an angle of $109.5^{\circ}$. This forms a tetragonal 
structure in three dimensions as shown in Figure 2.5. All the four bonds are strong sigma $(\sigma)$ bonds as seen in methane, which has four hydrogen atoms attached to a carbon atom, or in Diamond containing all carbon atoms.

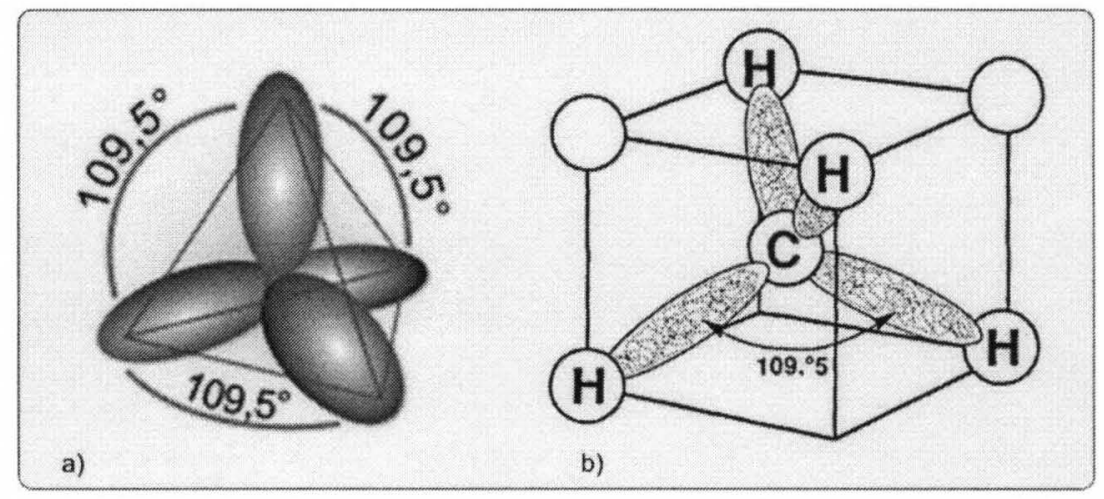

Figure 2.5 Schematic of $\mathrm{sp}^{3}$ hybridization. a) Four equivalent $\mathrm{sp}$ orbitals, in three dimensions as a tetragonal structure, form four strong $\sigma$ bonds resulting in $\mathrm{C}-\mathrm{C}$ (single bond between adjacent carbon atoms). b) $\mathrm{sp}^{3}$ bonding in methane. (adapted from reference [39-40]).

\subsection{Band structure of the electron states in graphene}

The graphene lattice consists of carbon atoms arranged in hexagonal pattern. Since adjacent carbon atoms (A and B) do not have identical environments, the structure is not really periodic [41], as shown in Figure 2.6. After lumping the two atoms together to form a unit cell, the lattice of unit cell becomes periodic. Every site has an identical environment for the unit. 

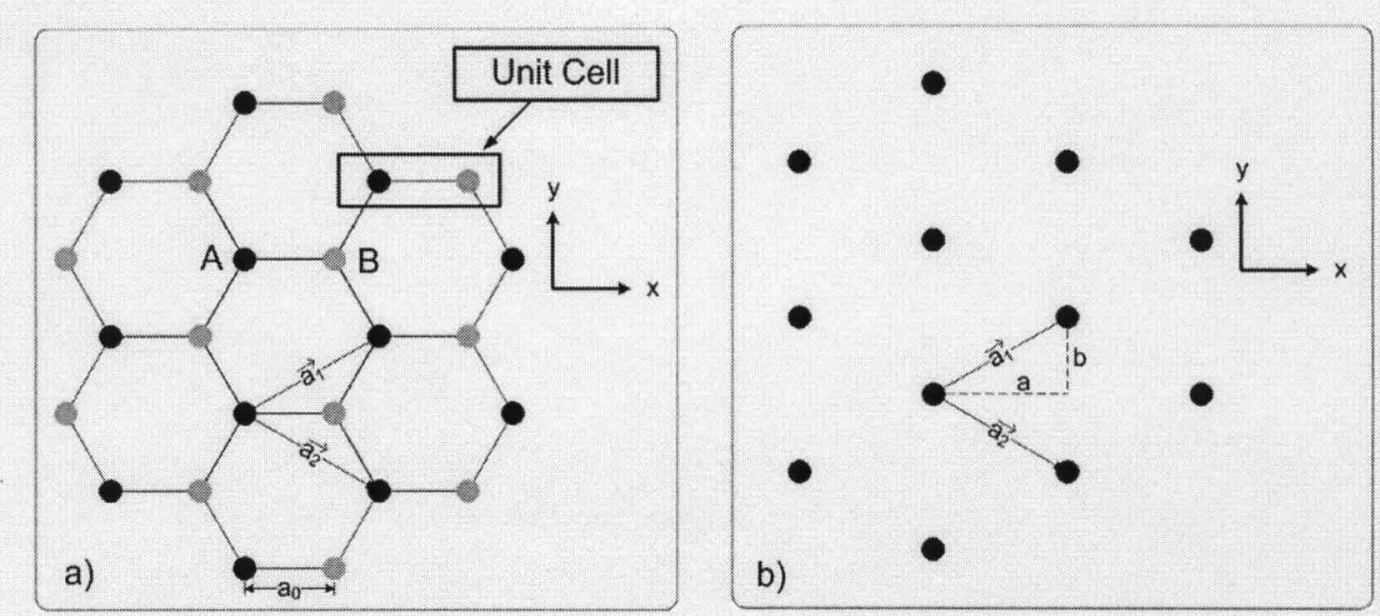

Figure 2.6 a) Graphene honeycomb structure showing the unit cell of two carbon atoms. b) Real space lattice showing the periodic arrangements of the unit cells with basis vectors $\overrightarrow{a_{1}}$ and $\overrightarrow{a_{2}}$.

Every point on this periodic lattice (formed by unit cells) can be described using two lattice vectors $\overrightarrow{a_{1}}, \overrightarrow{a_{2}}$ and a set of integers $(m, n)$ where

$\vec{R}=m \overrightarrow{a_{1}}+n \overrightarrow{a_{2}}$

with

$\overrightarrow{a_{1}}=\hat{x} a+\hat{y} b$

$$
\overrightarrow{a_{2}}=\hat{x} a-\hat{y} b
$$

where from Figure 2.6 a) and b),

$$
a=\frac{3}{2} a_{0} \quad b=\frac{\sqrt{3}}{2} a_{0}
$$

Using the basis vectors $\left(\overrightarrow{a_{1}}\right.$ and $\left.\overrightarrow{a_{2}}\right)$ reciprocal lattice basis vectors can be determined. In general, for three dimensions (3D) the points on the reciprocal lattice can be written as $\vec{K}=M \overrightarrow{A_{1}}+N \overrightarrow{A_{2}}+P \overrightarrow{A_{3}}$

Where $(\mathrm{M}, \mathrm{N}, \mathrm{P})$ are integers and $\overrightarrow{A_{1}}, \overrightarrow{A_{2}}, \overrightarrow{A_{3}}$ are determined such that $\overrightarrow{A_{j}} \cdot \overrightarrow{a_{\imath}}=2 \pi \delta_{i j}$ 
$\delta_{i j}$, being the Kronecker delta function (equal to 1 if $i=j$, and equal to zero if $i \neq j$ ).

$\overrightarrow{A_{1}}=\frac{2 \pi\left(\overrightarrow{a_{2}} \times \overrightarrow{a_{3}}\right)}{\overrightarrow{a_{1}} \cdot\left(\overrightarrow{a_{2}} \times \overrightarrow{a_{3}}\right)} \quad \overrightarrow{A_{2}}=\frac{2 \pi\left(\overrightarrow{a_{3}} \times \overrightarrow{a_{1}}\right)}{\overrightarrow{a_{2}} \cdot\left(\overrightarrow{a_{3}} \times \overrightarrow{a_{1}}\right)} \quad \overrightarrow{A_{3}}=\frac{2 \pi\left(\overrightarrow{a_{1}} \times \overrightarrow{a_{2}}\right)}{\overrightarrow{a_{3}} \cdot\left(\overrightarrow{a_{1}} \times \overrightarrow{a_{2}}\right)}$

For $2 \mathrm{D}$ situation, the vector $\overrightarrow{A_{3}}$ should be omitted and the vector $\overrightarrow{a_{3}}$ will become the unit vector $\hat{z}$. Therefore, for the graphene case the reciprocal lattice in the $k_{x}-k_{y}$ plane are given by

$\vec{K}=M \overrightarrow{A_{1}}+N \overrightarrow{A_{2}}$

and,

$\overrightarrow{A_{1}}=\frac{2 \pi\left(\overrightarrow{a_{2}} \times \hat{z}\right)}{\overrightarrow{a_{1}} \cdot\left(\overrightarrow{a_{2}} \times \hat{z}\right)}=\hat{x}\left(\frac{\pi}{a}\right)+\hat{y}\left(\frac{\pi}{b}\right)$

$\overrightarrow{A_{2}}=\frac{2 \pi\left(\hat{z} \times \overrightarrow{a_{1}}\right)}{\overrightarrow{a_{2}} \cdot\left(\hat{z} \times \overrightarrow{a_{1}}\right)}=\hat{x}\left(\frac{\pi}{a}\right)-\hat{y}\left(\frac{\pi}{b}\right)$

Using vectors $\overrightarrow{A_{1}}$ and $\overrightarrow{A_{2}}$ as the basis vectors, reciprocal lattice can be constructed as shown in Figure 2.7 [42]. The perpendicular bisectors to the reciprocal lattice vectors give the first Brillouin zone which depicts the range of $\mathrm{k}$ values over which unique values for the dispersion relation are defined.

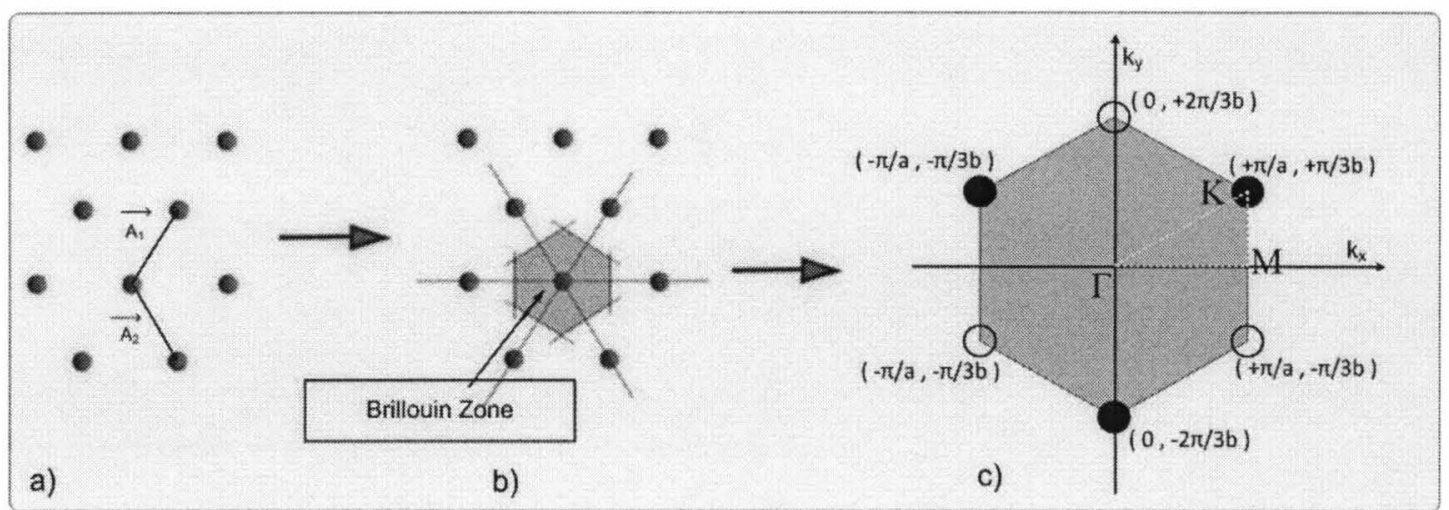

Figure 2.7 (a) Reciprocal lattice of graphene [42]. (b) Brillouin zone construction from the reciprocal lattice structure [42]. (c) Enlarged view of Brillouin zone, the coordinates for the six $\mathrm{K}$ points are also shown. Energy dispersion relations are obtained along the perimeter of the dotted triangle connecting the high symmetry points $\Gamma, \mathrm{K}$ and $\mathrm{M}$. 
Three high symmetry points $\Gamma, \mathrm{K}$ and $\mathrm{M}$ represent the center, corner and the center of an edge of the Brillouin zone respectively. The Figure 2.7 (c) shows the enlarged view of the first Brillouin zone having six equivalents $\mathrm{K}$ points (corners of the Brillouin zone) and their coordinates respectively.

Using matrix equation form, in general the band structure can be calculated by solving a matrix eigenvalue equation. Considering any unit cell $n$, (Figure 2.8 a [41]) from the lattice of a periodic solid, connected to its neighboring unit cells $m$ by a matrix $\left[H_{n m}\right]$ of size $(b \times b), b$ being the number of basis orbitals per unit cell, a matrix equation can be written as $E\left\{\phi_{n}\right\}=\sum_{m}\left[H_{n m}\right]\left\{\phi_{m}\right\}$

Where $\left\{\phi_{m}\right\}$ is a $(b \times 1)$ column vector denoting the wavefunction in unit cell $m$. Now from discrete version of Bloch theorm, $\left\{\phi_{m}\right\}=\left\{\phi_{0}\right\} e^{i \vec{k} \cdot \overrightarrow{d_{m}}}$, Which reduce the energy eigenvalue equation as $E\left\{\phi_{0}\right\}=[h(\vec{k})]\left\{\phi_{0}\right\}$ with, $[h(\vec{k})]=\sum_{m}\left[H_{n m}\right] e^{i \vec{k} \cdot\left(\overrightarrow{d_{m}}-\overrightarrow{d_{n}}\right)}$

The summation over $m$ runs over all neighboring unit cells (including itself) with which cell $n$ has any overlap (that is, for which $H n m$ is non-zero). The periodicity of lattice confirms that the result of the summation will be same for any unit cell $(n)$ chosen. 
The band structure can be plotted out by finding the eigenvalues of the $(b \times b)$ $\operatorname{matrix}[h(\vec{k})]$ for each value of $\vec{k}$ and it will have $b$ branches, one for each eigenvalue.

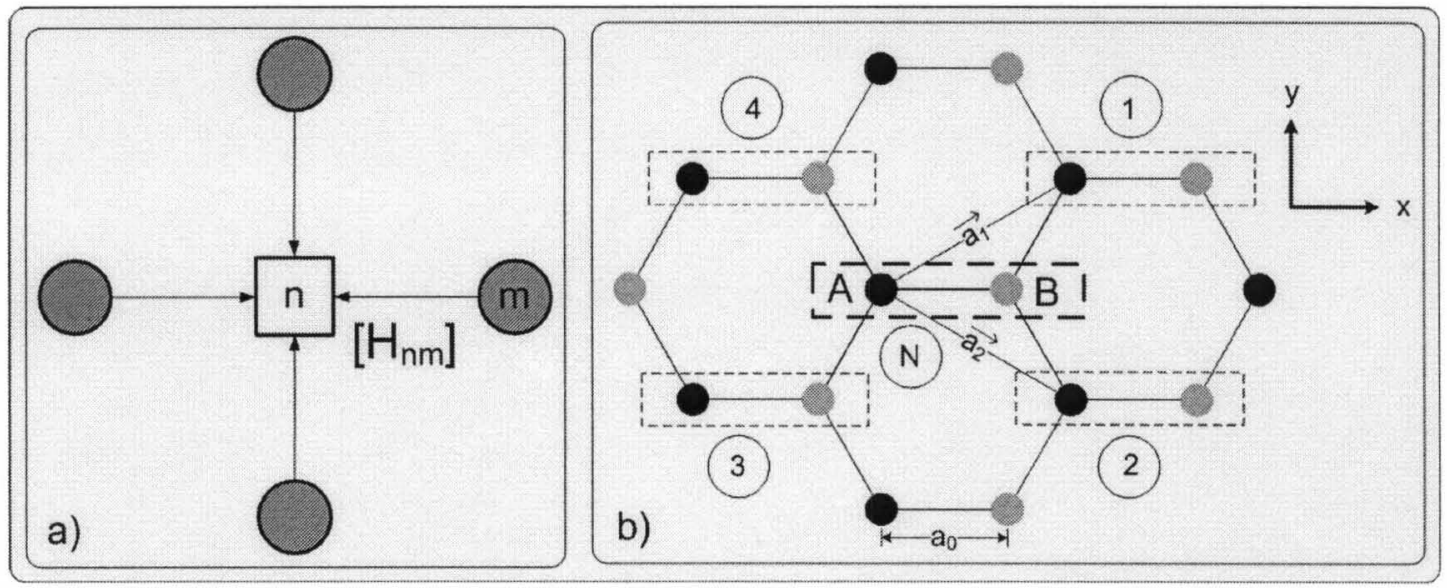

Figure 2.8 (a) Schematic showing a unit cell $n$ connected to its neighbor unit cells $m$ by a matrix [41]. (b) Schematic showing a unit cell $n$, of graphene having two carbon atoms A and $\mathrm{B}$, connected to its four neighbor unit cells (where $m=1,2,3,4$ ).

Now, for the graphene case due to the $\mathrm{sp}^{2}$ hybridization and delocalization of electrons in pi bonding system, the energy levels involving $2 \mathrm{~s}, 2 \mathrm{p}_{\mathrm{x}}, 2 \mathrm{p}_{\mathrm{y}}$ orbitals are decoupled from those involving $2 \mathrm{p}_{\mathrm{z}}$ orbitals. In other words, the levels that involve $2 \mathrm{~s}$, $2 p_{x}$ and $2 p_{y}$ orbitals are either too far below the Fermi level or too far above to influence the electrical conduction. This idea can be used to describe the valence and conduction bands by using one orbital per carbon atom resulting in a $(2 \times 2)$ matix $([h(\vec{k})])$. This matrix can be easily written by choosing any unit cell $(n)$ and summing over that unit cell and all its neighboring unit cells. Interaction between the neighboring carbon atoms (A on $\mathrm{B}$ or $\mathrm{B}$ on $\mathrm{A}$ ) is assumed to be $-\mathrm{t}$ while the interaction between same atoms that is $\mathrm{A}$ on $\mathrm{A}$ or $\mathrm{B}$ on $\mathrm{B}$ is taken to be zero (Figure $2.8 \mathrm{~b}$ ). 


$$
\begin{gathered}
{[h(\vec{k})]=\left[\begin{array}{ll}
n_{A} \rightarrow n_{A} & n_{A} \rightarrow n_{B} \\
n_{B} \rightarrow n_{A} & n_{B} \rightarrow n_{B}
\end{array}\right]+\left[\begin{array}{ll}
n_{A} \rightarrow 1_{A} & n_{A} \rightarrow 1_{B} \\
n_{B} \rightarrow 1_{A} & n_{B} \rightarrow 1_{B}
\end{array}\right]+\left[\begin{array}{ll}
n_{A} \rightarrow 2_{A} & n_{A} \rightarrow 2_{B} \\
n_{B} \rightarrow 2_{A} & n_{B} \rightarrow 2_{B}
\end{array}\right]} \\
+\left[\begin{array}{ll}
n_{A} \rightarrow 3_{A} & n_{A} \rightarrow 3_{B} \\
n_{B} \rightarrow 3_{A} & n_{B} \rightarrow 3_{B}
\end{array}\right]+\left[\begin{array}{ll}
n_{A} \rightarrow 4_{A} & n_{A} \rightarrow 4_{B} \\
n_{B} \rightarrow 4_{A} & n_{B} \rightarrow 4_{B}
\end{array}\right]
\end{gathered}
$$

Here, $\mathrm{n}$ is the unit cell chosen and $\mathrm{m}=1,2,3,4$ as shown in Figure 2.8. therefore

$$
\begin{aligned}
& {[h(\vec{k})]=\left[\begin{array}{cc}
0 & -t \\
-t & 0
\end{array}\right]+\left[\begin{array}{cc}
0 & 0 \\
-t e^{i \vec{k} \cdot \overrightarrow{a_{1}}} & 0
\end{array}\right]+\left[\begin{array}{cc}
0 & 0 \\
-t e^{i \vec{k} \cdot \overrightarrow{a_{2}}} & 0
\end{array}\right]+\left[\begin{array}{cc}
0 & -t e^{-i \vec{k} \cdot \overrightarrow{a_{1}}} \\
0 & 0
\end{array}\right]} \\
& +\left[\begin{array}{cc}
0 & -t e^{-i \vec{k} \cdot \overrightarrow{a_{2}}} \\
0 & 0
\end{array}\right] \\
& =>h(\vec{k})]=\left[\begin{array}{cc}
0 & -t\left(1+e^{-i \vec{k} \cdot \overrightarrow{a_{1}}}+e^{-i \vec{k} \cdot \overrightarrow{a_{2}}}\right) \\
-t\left(1+e^{i \vec{k} \cdot \overrightarrow{a_{1}}}+e^{i \vec{k} \cdot \overrightarrow{a_{2}}}\right) & 0
\end{array}\right] \\
& =>[h(\vec{k})]=\left[\begin{array}{cc}
0 & h_{0}^{*} \\
h_{0} & 0
\end{array}\right] \quad \text { where, } h_{0}=-t\left(1+e^{i \vec{k} \cdot \overrightarrow{a_{1}}}+e^{i \vec{k} \cdot \overrightarrow{a_{2}}}\right)
\end{aligned}
$$

The eigenvalues of the matrix are given by

$$
E(\vec{k})= \pm\left|h_{0}(\vec{k})\right|
$$

Recalling,

$$
\begin{aligned}
& \overrightarrow{a_{1}}=\hat{x} a+\hat{y} b=\hat{x} \frac{3}{2} a_{0}+\hat{y} \frac{\sqrt{3}}{2} a_{0} \\
& \overrightarrow{a_{2}}=\hat{x} a-\hat{y} b=\hat{x} \frac{3}{2} a_{0}-\hat{y} \frac{\sqrt{3}}{2} a_{0} \\
& \vec{k}=\hat{x} k_{x}+\hat{y} k_{y} \\
& h_{0}=-t\left(1+e^{i \vec{k} \cdot \overrightarrow{a_{1}}}+e^{i \vec{k} \cdot \overrightarrow{a_{2}}}\right)=-t\left(1+e^{i\left(k_{x} a+k_{y} b\right)}+e^{i\left(k_{x} a-k_{y} b\right)}\right) \\
& \quad=-t\left(1+2 e^{i k_{x} a} \cos k_{y} b\right) \\
& =>\left|h_{0}\right|^{2}=h_{0} h_{0}^{*}=t^{2}\left(1+4 \cos k_{x} a \cos k_{y} b+4 \cos ^{2} k_{y} b\right)
\end{aligned}
$$

So,

$$
E(\vec{k})= \pm\left|h_{0}(\vec{k})\right|= \pm t \sqrt{1+4 \cos k_{x} a \cos k_{y} b+4 \cos ^{2} k_{y} b}
$$


or in terms of $\mathrm{a}_{0}$ (graphene lattice constant)

$$
E(\vec{k})= \pm t \sqrt{3+4 \cos \left(\frac{3}{2} k_{x} a_{0}\right) \cos \left(\frac{\sqrt{3}}{2} k_{y} a_{0}\right)+2 \cos \left(\sqrt{3} k_{y} a_{0}\right)}
$$

The above equation gives the energy dispersion relation for 2D graphite as depicted in Figure 2.9. Here "t" is called the transfer integral (it value lies between 2.5 to 3 ). Two eigenvalues were achieved (one positive and one negative) for each value of $\vec{k}$ resulting in two branches in the $E(\vec{k})$ plot as shown in Figure $2.9[36,43]$. The upper half represents the $\pi^{*}$ anti-bonding energy band (conduction band) and the lower half is the $\pi$ bonding energy band (valence band).

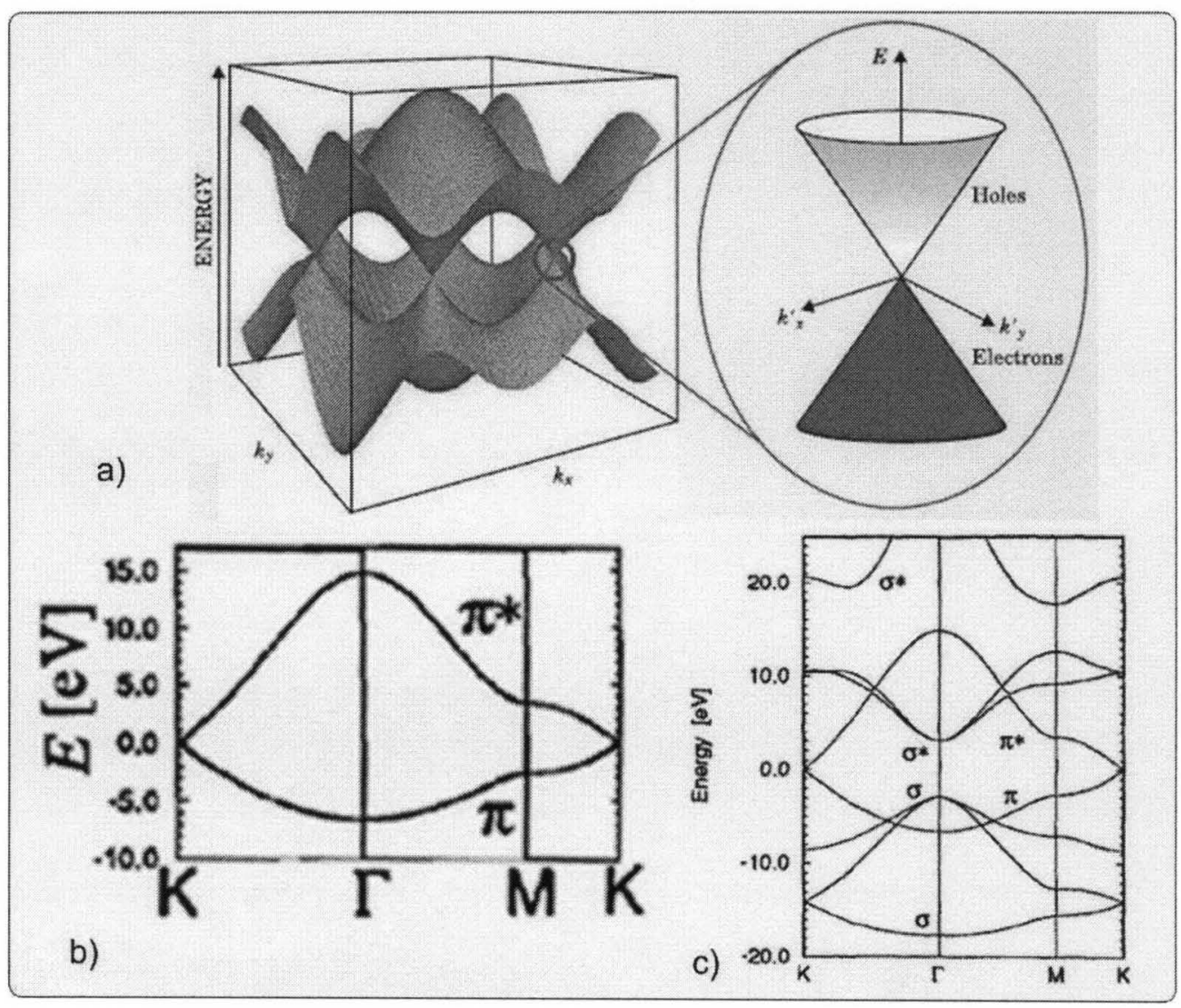

Figure 2.9 (a) Energy dispersion of graphene. b) The dispersion of $\pi / \pi^{*}$ bands along the high symmetry directions $\Gamma, \mathrm{M}, \mathrm{K}$. c) Dispersion of all the $\sigma / \sigma^{*}$ and $\pi / \pi^{*}$ bands along $\Gamma$, $\mathrm{M}, \mathrm{K}$ high symmetry directions (adapted from [36, 43]). 
As seen in the Figure $2.9, \pi$ bonding and $\pi^{*}$ anti-bonding bands are degenerate for some values of $\mathrm{k}$ at the Fermi energy $(\mathrm{E}=0)$. To locate these points:

$|h(\vec{k})|=0$

$\left|h_{0}(\vec{k})\right|= \pm t \sqrt{1+4 \cos k_{x} a \cos k_{y} b+4 \cos ^{2} k_{y} b}=0$

Assuming $\mathrm{k}_{\mathrm{x}} \mathrm{a}=0$ and calculating $h(\vec{k})$ as a function of $\mathrm{k}_{\mathrm{y}}$

$h_{0}=t\left(1+2 \cos k_{y} b\right)=>$ to get $h_{0}(k)=0 ; k_{y} b=\frac{2 \pi}{3}$

For $\mathrm{k}_{\mathrm{x}} \mathrm{a}=\pi$

$h_{0}=t\left(1-2 \cos k_{y} b\right)=>$ to get $h_{0}(k)=0 ; k_{y} b=\frac{\pi}{3}$

Therefore, the coordinates of six points (corners) for which $h_{0}(k)=0$ in the Brillouin zone are $(0,2 \pi / 3 b),(0,-2 \pi / 3 b),(\pi / a, \pi / 3 b),(\pi / a,-\pi / 3 b),(-\pi / a, \pi / 3 b)$ and $(-\pi / a,-\pi / 3 b)$ as shown in Figure 2.8. All these points show zero band zap conduction valley in graphene bandstructure. The density of states for graphene is calculated from the electron dispersion relation and as shown in Figure 2.10. The DOS of graphene can be approximated to linear behavior near the Fermi energy. Two peaks one each in $\pi$ bonding and $\pi^{*}$ anti-bonding can be seen.

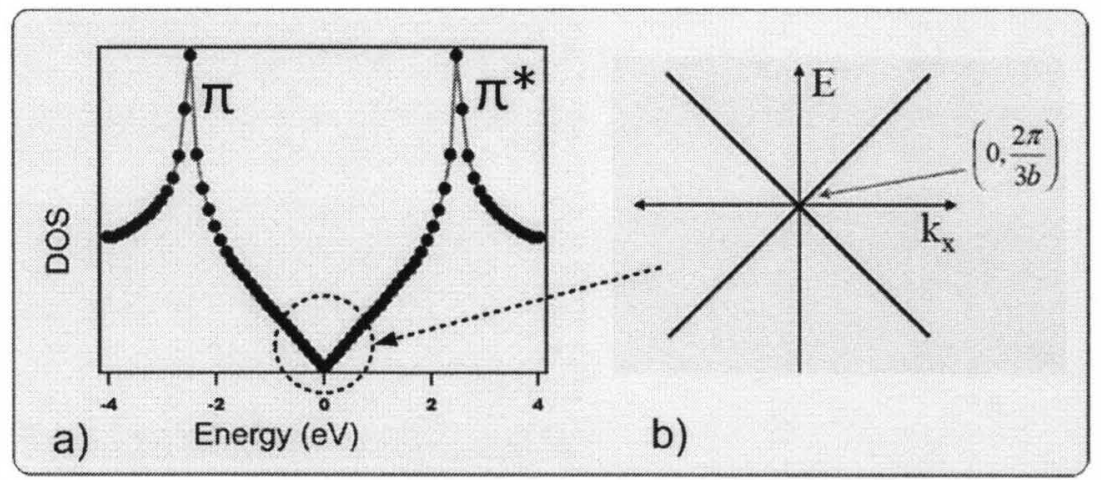

Figure 2.10 (a) Density of States (DOS) of. b) DOS shows linear behavior near corners where both the conduction and valence band meet. 


\subsection{Phonon Dispersion in graphene}

To interpret the Raman spectra of graphene samples, understanding the phonon dispersion of graphene is essential. In the graphene crystal structure, there are two carbon atoms per unit cell resulting in six phonon modes with different dispersion relations. Three of the six phonon modes are acoustic $(A)$ and the other there are optic $(\mathrm{O})$ phonon modes. The phonon modes propagating in the direction of $\mathrm{C}-\mathrm{C}$ bond between the two carbon atoms (A, B) in the graphene unit cell are marked as longitudinal (L) mode while the modes travelling perpendicular to longitudinal mode are labeled as transverse (T) modes. Two out of three phonon modes, for both acoustic and optic phonons, are in-plane (i) modes while one mode for each (acoustic and optic) is out of plane (o). As a result, the six phonon dispersion curves are assigned to iLA (or LA), iTA and oTA for the acoustic branch and iLO (or LO), iTO and oTO for the optical branch [36, 44-46].

The iLO and iTO phonon modes, corresponding to the vibrations of the sublattice A against the sublattice $\mathrm{B}$, are degenerate at the $\Gamma$ point (zone center). The degeneracy of these modes disappears for points inside the first Brillouin zone. Group theory tells us that the degenerate LO and iTO phonon modes ( $E_{2 g}$ symmetry) at the $\Gamma$ point are Raman active, while the oTO phonon mode is infrared active. The phonon modes in the vicinity of the $\mathrm{K}$ point are related to the D-band and the $\mathrm{G}^{\prime}$-band in the Raman spectra [47]. At the K-point, the phonon which comes from the iTO branch is non-degenerate while The LO and LA phonon branches meet each other at the $\mathrm{K}$ point giving rise to a doubly degenerate phonon. The phonon mode frequencies of graphite have been recently observed by inelastic X-ray scattering [48] as shown in Figure 2.11. 


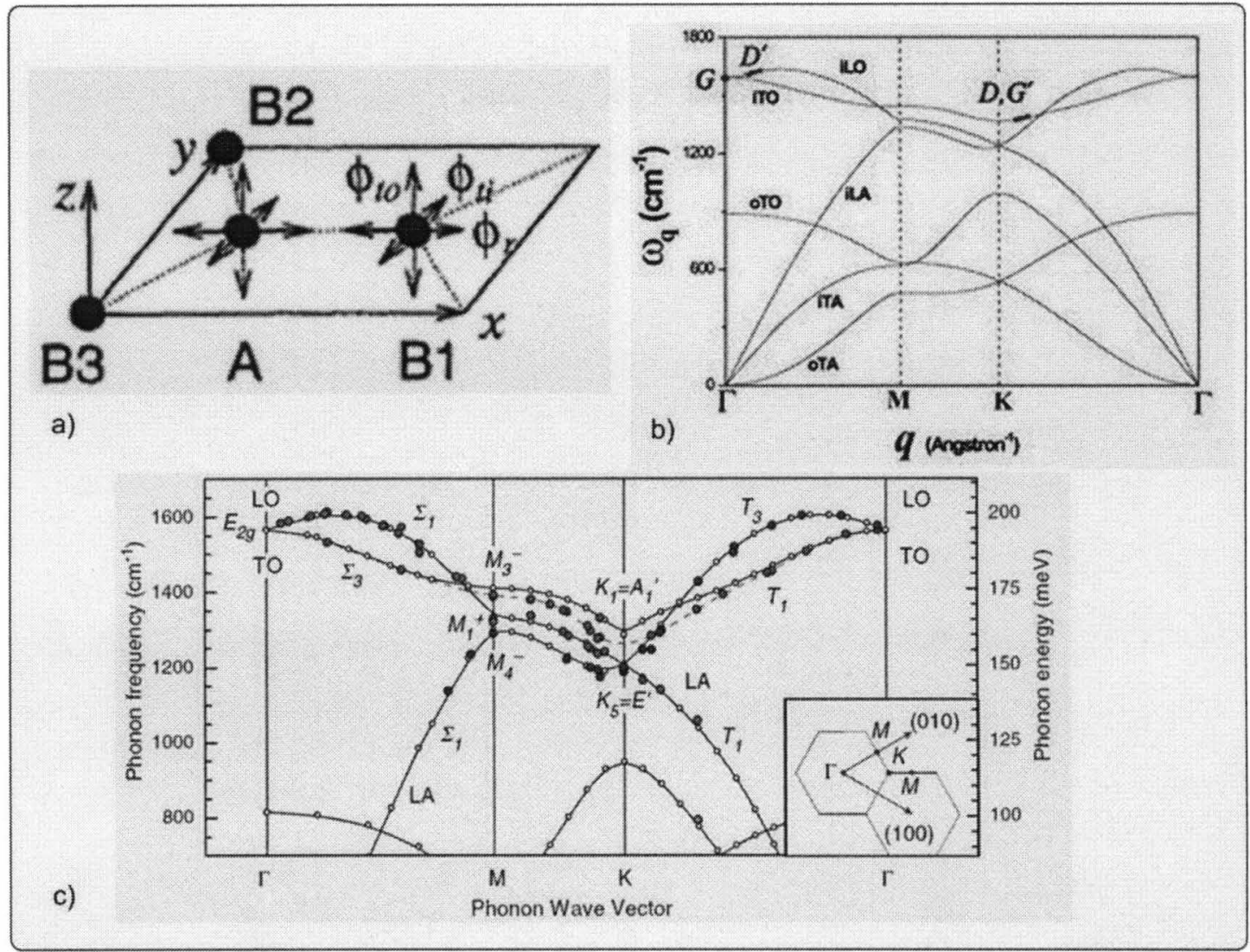

Figure 2.11 (a) Force constants between the A and B1 carbon atoms on a graphene sheet. Here, $\phi_{\mathrm{r}}, \phi_{\mathrm{ti}}, \phi_{\mathrm{to}}$ represents forces from the nearest neighbor atoms in the radial, bond stretching, (in-plane longitudinal modes, iLA, iLO), in-plane (iTA, iTO) and out of plane tangential (oTA, oTO) bond bending directions respectively. [36] (b) Calculated phonon dispersion curves plotted along high symmetry directions for a $2 \mathrm{D}$ graphene sheet. Phonon modes and their location along the high symmetry direction representing four features in the Raman spectra (D, G, D' and G') are also highlighted [47]. (c) Inelastic Xray measurements and ab initio calculations of phonon dispersion curves in graphite (filled dots represent experimental data) [48].

\subsection{Summary}

In graphene every carbon atom is $\mathrm{sp}^{2}$ hybridized providing it a planar hexagonal structure (carbons atom $120^{\circ}$ apart from each other). The electrons in the three sigma $(\sigma)$ bonds are tightly bound within the planar geometry while the electrons in the pi $(\pi)$ bonds 
delocalized over the entire graphene sheet. Resultant reduced coupling among the electrons from $\sigma$ bonds with the electrons in pi bond system allows the density of states of graphene to be governed solely by the pi electrons (energy bands due to the contribution of electrons in the $\sigma$ bonds are far away from the Fermi energy).

As seen in the density of states of graphene (Figure 2.10), the conduction band and the valence band meet at Fermi energy forming a zero-band gap semiconductor. The energy dispersion is linear near the zero-band gap points (corners of the Brillouin zone) and two sharp peaks symmetric to the Fermi energy are observed, one each, in $\pi$ bonding and $\pi^{*}$ anti-bonding.

The sigma $(\sigma)$ bonds govern the mechanical properties of the graphene sheet, giving it a high Young's modulus and high tensile strength and making it the strongest 2D material know until now. The pi $(\pi)$ electrons provide graphene with its exceptional current carrying capacity at very high speeds resulting in large carrier mobilities.

The two atom unit cell in graphene results in six modes of vibration in the structure, three acoustic modes and three optical modes. The optical modes of the phonons in graphene allow for an understanding of the Raman spectra features. These Raman features can be used to indicate the quality of the graphene and the number of graphene layers. The difference in the Raman features can provide a quick way to differentiate among graphene, GO and rGO samples.

The next chapter will provide a summary of the most common methods of synthesis of the three materials (graphene, graphene oxide and reduced graphene oxide). The second part describes the differences in the electrical and optical properties of these materials. 


\section{CHAPTER III}

\section{SYNTHESIS and CHARACTERIZATION}

\subsection{Graphene Synthesis Techniques}

Synthesis of graphene can be categorized broadly in two major approaches: extracting single-or-few layers from bulk material and growing graphene layer by layer. In the first approach graphene layers in the bulk material are exfoliated and extracted and then deposited or dispersed on the desired substrate. In the second approach, the substrate is used as a catalytic material to grow graphene mono-or-few layers and these are then transferred to the desired substrates.

Since graphite is a series of stacked parallel layers of graphene, it is an obvious starting material to extract graphene. A schematic of graphite is shown in Figure 3.1. Within each layer, the carbon atoms form three covalent bonds (sigma, $\sigma$ ) having short length $(0.142 \mathrm{~nm})$ and high strength $(524 \mathrm{~kJ} / \mathrm{mole})$ [39]. The fourth valence electron is paired with another delocalized electron of the adjacent layer by a much weaker van der Waals bond (a bond arising from structural polarization) only $7 \mathrm{KJ} / \mathrm{mole}$ in strength [39]. The spacing between the layers is relatively large $(0.335 \mathrm{~nm}$, more than twice the spacing between atoms in the basal plane) and approximately twice the van der Waals radius for carbon atom. In graphite, the delocalized electrons can move readily from one side of the 
planar layer to the other but cannot easily move from one layer to another resulting in an anisotropy ratio of bond strength (bonds in basal plane as compared to bonds between adjacent layers) of approximately 75 . This means that, while the bond strength in the basal plane is considerable, the bond strength in the c direction (interlaminar strength) is low and graphite shears easily between basal planes. The interplanar distance is further increased by lattice defects, interstitial foreign atoms and other irregularities. As a result the basal planes can slip easily over each other without loosing their coherence, and graphite is able to yield plastically.

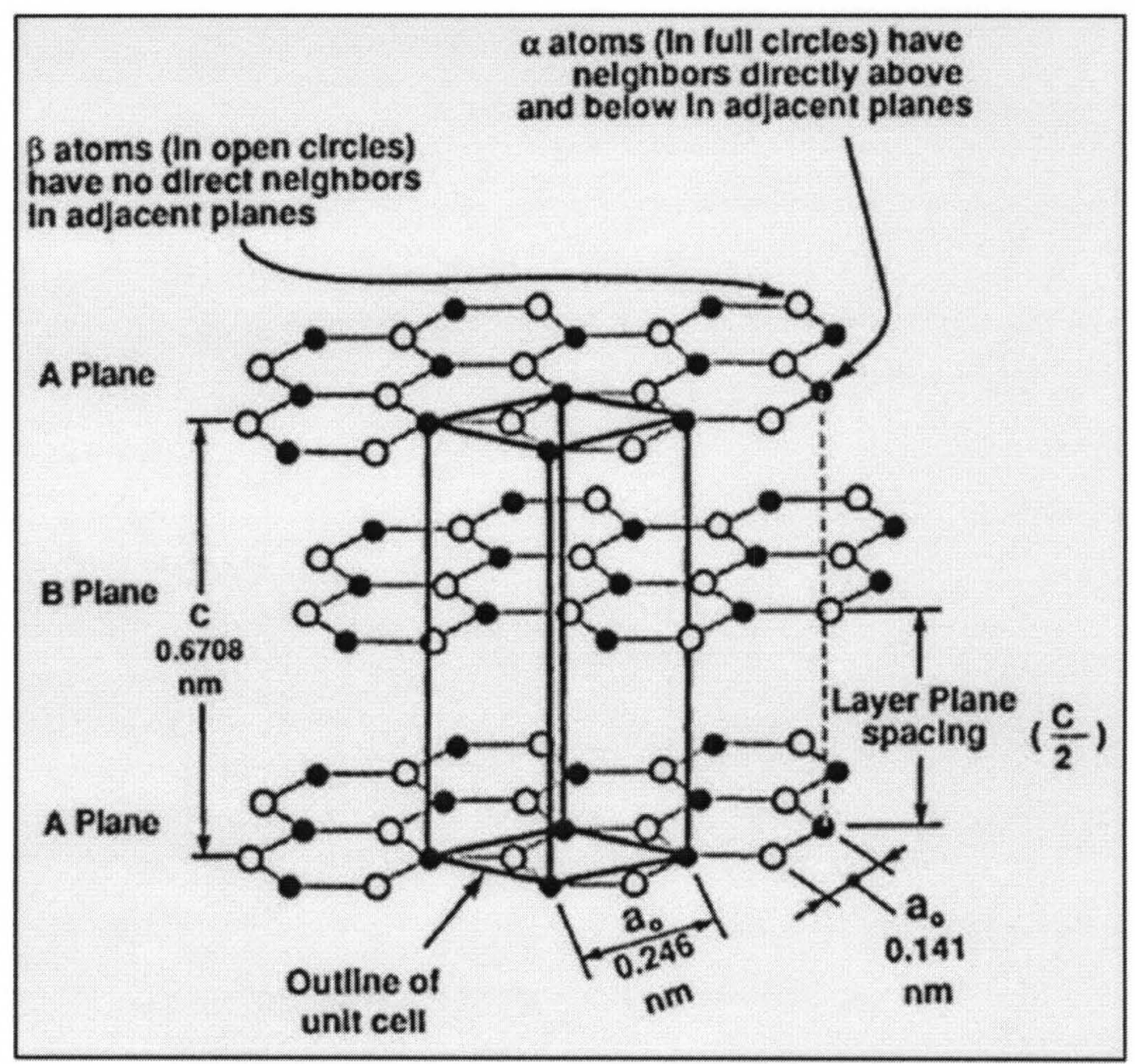

Figure 3.1 Schematic of crystal structure of graphite showing ABAB stacking sequence and unit cell (adapted from [39]). 


\subsubsection{Mechanical exfoliation technique (scotch tape method)}

The mechanical exfoliation of graphite to shear away multiple layers of graphene has been done for centuries. As a writing material, graphite (in chalk or pencils) spread as a large number of graphene sheets of varying thickness over the writing area. However, this method generates thick multilayer pieces and locating an isolated single layer of graphene is a difficult problem.

Improving on this approach in 1999, Prof. Rodney Ruoff reported a method of rubbing the tiny pillars of graphite against the Si substrate to produce thin graphite disks [49]. This method was again limited, however by the thickness of the resultant pieces. Later, Prof. Philip Kim dragged a graphite crystal mounted to an AFM tip along the surface of the substrate to separate graphite flakes from the bulk graphite [50]. The thickness of the separated flakes was still around 10nm ( 30-40 layers) and locating thinner flakes remained difficult. In 2004, Prof. Andre Geim developed an innovative technique of micro-mechanical cleavage of bulk graphite, into very thin sheets including mono-layers, with adhesive tape $[8,33,51]$. Also, they discovered a way to identify monolayers of graphene on $\mathrm{SiO}_{2}$ substrates (having specific thickness of either $100 \mathrm{~nm}$ or $300 \mathrm{~nm}$ ) by utilizing the difference in the interference contrast between bare substrate and graphene. In other words, a mono layer of graphene lying on $100 \mathrm{~nm}$ or $300 \mathrm{~nm} \mathrm{SiO}_{2} / \mathrm{Si}$ substrate produces a feeble interference contrast in an optical microscope with respect to the bare substrate which allows its identification among the sea of bulk graphite pieces. This optical method of locating monolayer is very sensitive to the thickness of the oxide 
layer; a mere $10 \%$ change in oxide thickness can make the graphene layers invisible again.

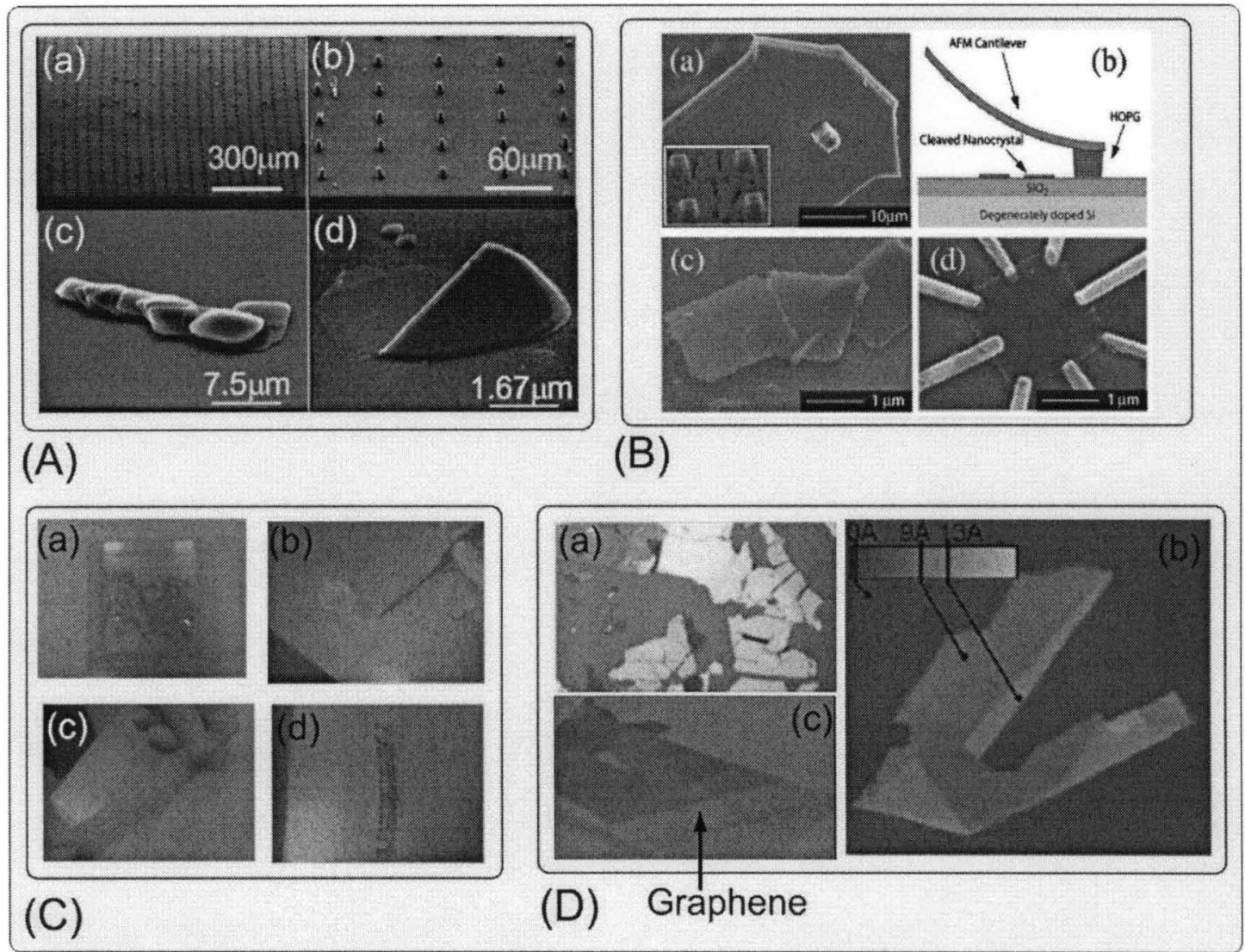

Figure 3.2 (A) Dr. Ruoff's approach of rubbing tiny pillars of graphite against Si. (a) HOPG sample, (b) Pillars formed on HOPG by oxygen plasma etching, (c) \& (d) examples of deposited graphene pieces [49]. (B) Dr. Philip method of attaching graphite to AFM tip.(a) SEM image of AFM with graphite piece, (b) Schematic showing AFM tip with HOPG touching the substrate, (c) deposited graphene flakes, (d) device fabricated using one of the flakes [50]. (C) Process of scotch tape deposition. (a) Graphite flakes, (b) Flakes were transferred to scotch tape using tweezers, (c) Pealing the sheets from the initial flake using second scotch tape, (d) Scotch tape with many thin graphene flakes. (D) Images of graphene pieces obtained by scotch tape method. (a) Optical image (lower magnification), (b) AFM image of graphene piece, (c) Optical image at higher magnification showing single graphene sheet $[33,51]$.

This technique simplifies the process of location of graphene pieces, however, provides no control on the size of the graphene pieces produced. This precludes the possibility of fabricating graphene devices on a large scale and limits its use for research 
purposes. Also, the peeling of the layers was performed manually in an uncontrolled fashion. This leads to different degrees of applied stress on different layers and cause different amounts of vertical displacements leading to huge variation in thickness of graphene pieces.

\subsubsection{Chemical exfoliation technique (from GO to graphene)}

Chemical exfoliation techniques follow three general steps: intercalation of graphite material, expansion/exfoliation of graphite layers and dispersion of separated layers in a solvent with subsequent deposition on to suitable substrate [52]. Intercalation is a process whereby an intercallant material is inserted between the graphene layers of a graphite crystal using chemical reactions. The most common form of intercalation is by oxidizing the graphite material with strong acids [53-54]. Since the interaction between the adjacent layers of graphite is weak, oxidizing agents move in the gap between the layers and are trapped. If these intercallant particles are somehow rapidly removed, while escaping they increase the separation between the layers and convert the graphite into a fluffy material. Rapid heating is the most common way of expanding and removing the intercallant particles [53]. As the intercalated graphite is heated rapidly to very high temperatures $\left(>1000^{\circ} \mathrm{C}\right)$ the intercallant particles converts to a gaseous form which results in an immense increase in their volume. The pressure generated by this volume increase forces the adjacent graphene layers to separate. Since the expansion process depends on harnessing the force developed by an expanding gas, sudden gas expansion is required. During slow heating, gas molecules have more time to escape resulting in less pressure 
build up and hence in little or no expansion. The expanded graphite flake material can be sonicated in solution to achieve separate layers dispersed in the solvent.

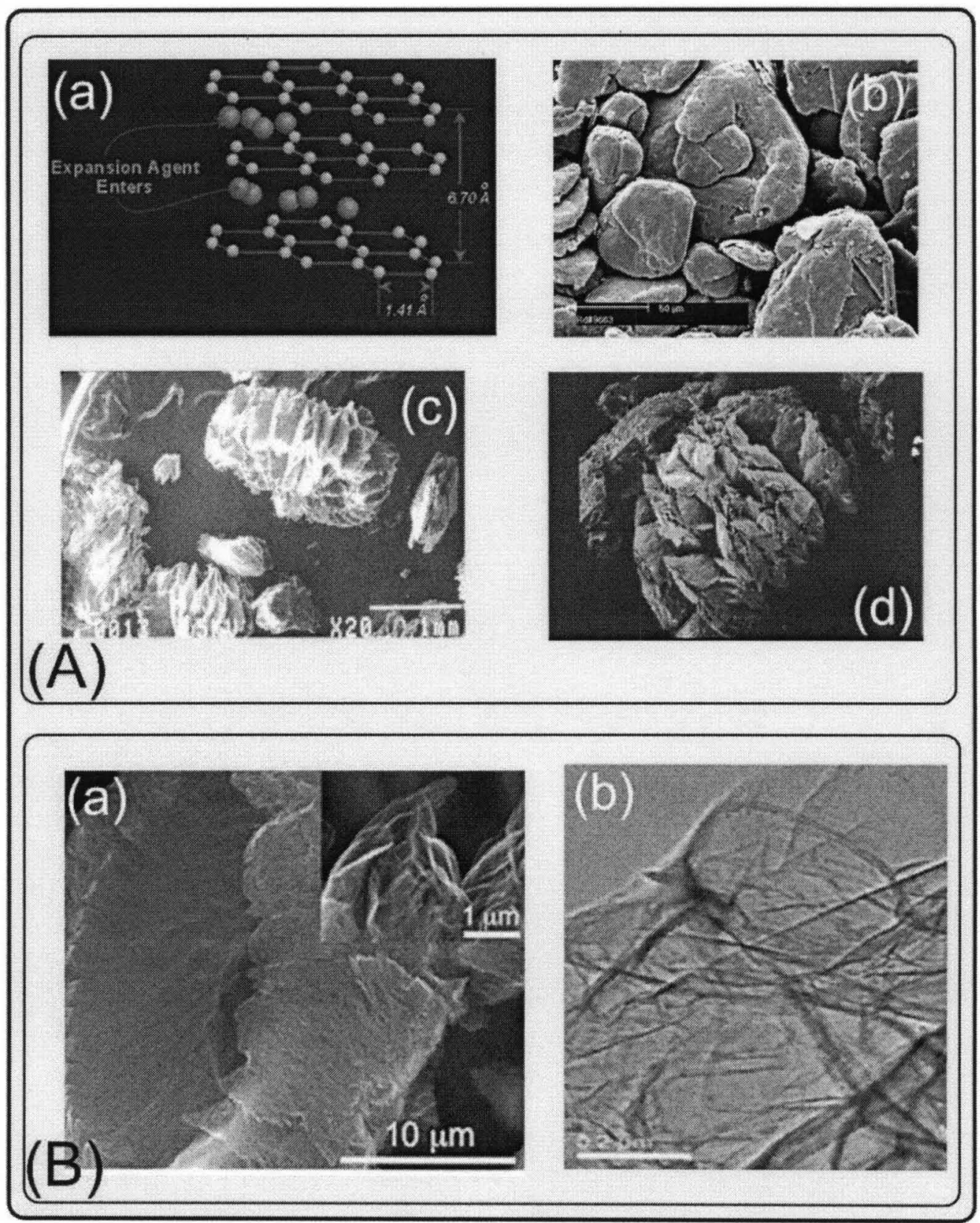

Figure 3.3 (A) (a) Schematic of intercalated material between the graphite layers, (b) Unexpanded intercalated graphite material, (c) \& (d) Expanded/ exfoliated graphite flakes. (B) Microwave assisted graphene exfoliation. (a) Exfoliated graphite. (b) SEM image of the dispersed graphene sample on the substrate. ( adapted from (A) [52], (B) [55]). 
Different approaches have been followed both for intercalation and expansion. Rapid heating and microwave treatment [55] followed by high frequency sonication and centrifugation is employed by various groups. The main advantage of this method is the bulk production of graphene sample. The disadvantage of this process is that many of the graphene layers end up bundling again while being deposited on the required substrate resulting in wrinkles and folds. Secondly, many deposited graphene pieces will be attached by intercalate compounds which results in modifications to its physical and chemical properties.

\subsubsection{Epitaxial growth on silicon carbide}

The mechanical and chemical exfoliation of bulk graphite to obtain graphene provides a fast way to produce samples for the study of the physical and electrical properties of single or few layer graphene, but it lacks the control on the location and thickness of the deposited graphene layer. Epitaxial growth of high quality graphene on substrates offers the control on the location of layers and partial control on the thickness of the layers. Graphene multilayers are grown epitaxially on single crystal silicon carbide (SiC) substrate. When crystals of $4 \mathrm{H}-$ or $6 \mathrm{H}-\mathrm{SiC}$ are heated to about $1300^{\circ} \mathrm{C}$ in ultra high vacuum (UHV) graphitic films are grown [56]. Epitaxial multilayer graphene films have been extensively characterized by surface analysis tools, electronic transport experiments and optical spectroscopy techniques. Figure 3.4 shows the STM image of few layer graphene grown on the Si terminated (0001) face of single-crystal 6H-SiC. 


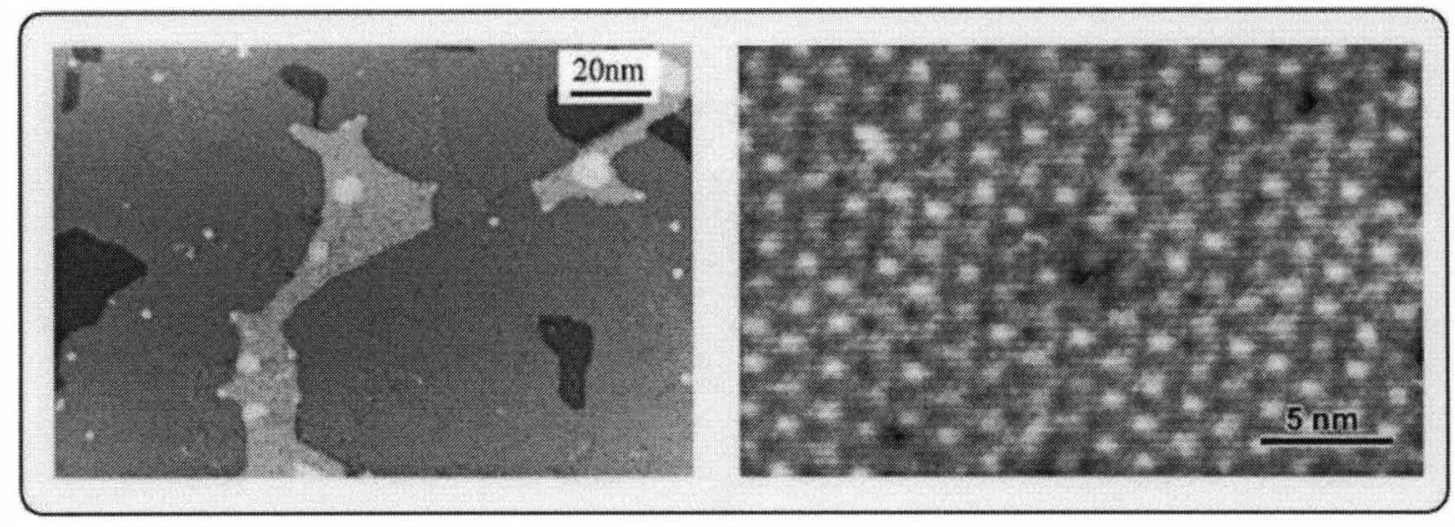

Figure 3.4 STM topographs of epitaxial graphene on SiC (0001). [56]

The face of the SiC wafer used (Si terminate or C terminate) influences the thickness, mobility and the carrier density of the graphene grown. Epitaxial growth of graphene provides high quality graphene layers; however, the process is limited to $\mathrm{SiC}$ substrate which reduces the impact of this method. This method allows growth of uniformly thick layers and provides limited control (by controlling the temperature and time of growth) on the number of layers grown.

\subsubsection{CVD growth on metal substrates}

The chemical vapor deposition (CVD) method involves the use of a metal layer as a seed for the growth of graphene. The solubility of carbon in the metal determines the number of layers grown. Ruthenium was one of the first metals to be used for CVD growth of graphene. Many metals have been used for growing graphene; however, uniform large scale growth of high quality monolayer graphene is still a challenge to overcome. Graphene grown on nickel $(\mathrm{Ni})$, due to its small grain boundaries and high carbon solubility, produces multilayer graphene. Recently, copper has emerged as a good 
choice of metal for CVD growth. Under low pressure conditions graphene growth on copper foil stops after forming single layer of graphene; however at atmospheric pressure copper produces multilayer graphene similar to that seen for nickel.

The growth of monolayer graphene by CVD technique is explored by many groups [57 -58] resulting in varying growth conditions and transfer procedures thereafter. The following section list the overall procedure and the CVD growth conditions employed in this dissertation.

CVD growth on copper ( $\mathrm{Cu})$ foil: Copper foil samples (25 $\mu \mathrm{m}$ thick and $2 \mathrm{~cm} \times 2 \mathrm{~cm})$ were dipped into acetic acid for 10 minutes to remove any natural occurring $\mathrm{Cu}$ oxide. After 10 minutes of treatment the samples were dried with compressed air and placed in a quartz tube CVD furnace and pumped using a mechanical pump. The temperature is raised to $1000{ }^{\circ} \mathrm{C}$ in steps of 100 with a flow of $2 \mathrm{sccm} \mathrm{H}_{2}$ and $18 \mathrm{sccm}$ Ar while maintaining a pressure of $100 \mathrm{mTorr}$. Samples are then annealed at $1000{ }^{\circ} \mathrm{C}$ for 30 minutes while maintaining the $\mathrm{H}_{2} / \mathrm{Ar}$ flow. After 30 minutes annealing, as the carbon feed for the graphene growth, $10 \mathrm{sccm}$ of Methane $\left(\mathrm{CH}_{4}\right)$ is added to the previous gas mixture of $2 \mathrm{sccm}$ of $\mathrm{H}_{2}$ and $18 \mathrm{sccm}$ of Ar changing the pressure to $500 \mathrm{mTorr}$ for 15 minutes (growth time). The furnace is cooled down slowly while maintaining the gas flow and the pressure.

After growth the graphene is transferred to the desired substrate following PMMA transfer approach. A thick Layer of PMMA is spun over the top side of the $\mathrm{Cu}$ foil (500 $\mathrm{rpm}$ for $16 \mathrm{sec}$ ) and cured at $180^{\circ} \mathrm{C}$ for 2 minutes. Backside $\mathrm{Cu}$ is etched overnight using ferric chloride solution. After the $\mathrm{Cu}$ is etched the PMMA supported graphene is transferred to DI water. After 2 hours the sample is transferred into a fresh DI water 
container and sonicated for 1 minute. The PMMA/graphene sample is then transferred onto the desired substrate (graphene side in contact with the substrate). A drop of PMMA is added onto the graphene/PMMA piece to relax the graphene which helps in reducing the ripples in graphene film during transfer. PMMA is then washed away with acetone while the graphene piece stays on the substrate.

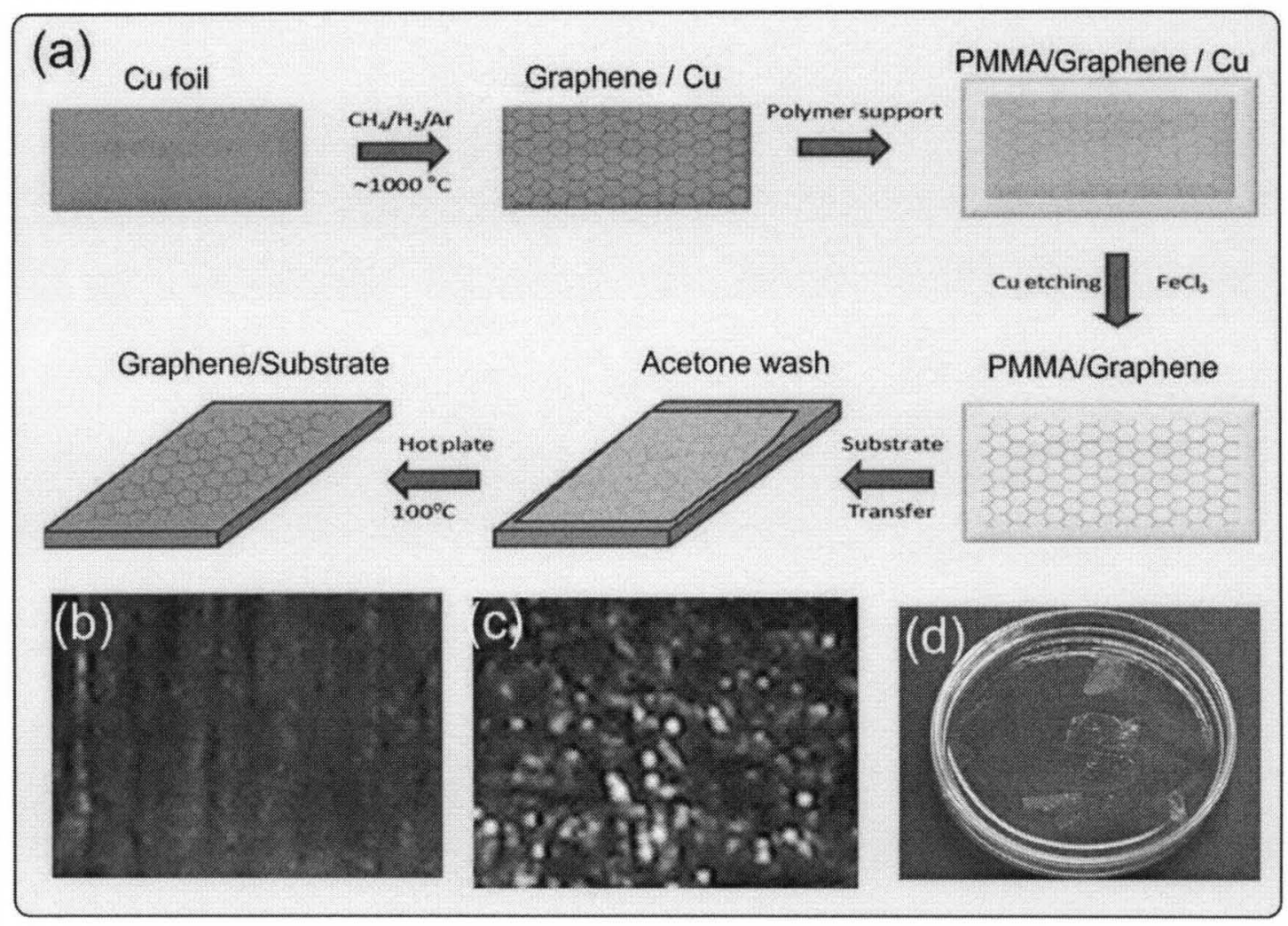

Figure 3.5 (a) Schematic of the CVD growth process flow. (b) $\mathrm{Cu}$ foil before growth. (c) $\mathrm{Cu}$ foil after growth. (d) PMMA/Graphene samples flowing on DI water.

\subsection{Graphene Oxide (GO) Synthesis Techniques}

Oxidation of graphene follows the processes used for preparing graphite oxide (also known as graphitic acid). Hummers method, Brodie's method and Staudenmaier's method for making graphite oxide were followed as in the start. Brodie and Staudenmaier 
used a combination of potassium chlorate $\left(\mathrm{KClO}_{3}\right)$ with nitric acid $\left(\mathrm{HNO}_{3}\right)$ to oxidize graphite, while the Hummers method involves treatment of graphite with potassium permanganate $\left(\mathrm{KMnO}_{4}\right)$ and sulfuric acid $\left(\mathrm{H}_{2} \mathrm{SO}_{4}\right)$ [59]. However, they all result in nonuniform oxidation of graphene. Recently a modified Hummers method [60] which involves a pre-oxidation step is being followed. The following section provides the details of the modified Hummers method followed in this dissertation.

Modified Hummers Method [61]: Graphite oxide is synthesized from graphite powder (SP-1 grade 325 mesh, Bay Carbon Inc.). The graphite is first pretreated in order to fully oxidize it to graphite oxide (GO). To accomplish this concentrated $\mathrm{H}_{2} \mathrm{SO}_{4}(50 \mathrm{ml})$ is heated to $90{ }^{\circ} \mathrm{C}$ in a $300 \mathrm{ml}$ beaker with $\mathrm{K}_{2} \mathrm{~S}_{2} \mathrm{O}_{8}(10 \mathrm{~g})$ and with $\mathrm{P}_{2} \mathrm{O}_{5}(10 \mathrm{~g})$ added with stirring until all of the reactants are completely dissolved. The mixture is then cooled to $80^{\circ} \mathrm{C}$. Graphite powder $(12 \mathrm{~g})$ is then added to the $\mathrm{H}_{2} \mathrm{SO}_{4}$ solution resulting in bubbling which subsides within 30 minutes. The mixture is kept at $80^{\circ} \mathrm{C}$ for 4.5 hours using a hotplate after which the heating is stopped and the mixture diluted with $2 \mathrm{~L}$ of DI water and left overnight. The following day the mixture is filtered and washed using a 0.2 micron Nylon Millipore filter to remove all traces of acid. The solid is transferred to a drying dish and allowed to dry in air overnight. For the oxidation step of the synthesis, $\mathrm{H}_{2} \mathrm{SO}_{4}(460 \mathrm{ml})$ is placed into a $2 \mathrm{~L}$ Erlenmeyer flask and chilled to $0{ }^{\circ} \mathrm{C}$ using an ice bath. The pretreated graphite is then added to the acid and stirred. $\mathrm{KMnO}_{4}(60 \mathrm{~g})$ is added slowly and allowed to dissolve with the aid of stirring, while the temperature is closely monitored so as not to allow the mixture to go above $10^{\circ} \mathrm{C}$. This mixture is then allowed to react at $35^{\circ} \mathrm{C}$ for 2 hours after which distilled water $(920 \mathrm{ml})$ is added, initially in $20-30 \mathrm{ml}$ aliquots. Since the addition of the water causes the temperature of 
the mixture to rise rapidly, water addition is carried out in an ice bath so that the temperature does not climb above $50{ }^{\circ} \mathrm{C}$. As more water is added, the mixture becomes less reactive until the final $\sim 700 \mathrm{ml}$ can be poured in with no observable resulting rise in temperature. After adding all of the $920 \mathrm{ml}$ of DI water, the mixture is stirred for 2 hours at which time $2.8 \mathrm{~L}$ of DI water is added. Shortly after the dilution with $2.8 \mathrm{~L}$ of water, $50 \mathrm{ml}$ of $30 \% \mathrm{H}_{2} \mathrm{O}_{2}$ is added to the mixture resulting in a brilliant yellow color along with bubbling. The mixture is allowed to settle for at least a day after which the clear supernatant is decanted. The remaining mixture is centrifuged and washed with a total of $5 \mathrm{~L}$ of $10 \% \mathrm{HCl}$ solution followed by $5 \mathrm{~L}$ of DI water to remove the acid. The resulting solid is dried in air and diluted to make a $2 \% \mathrm{w} / \mathrm{w}$ dispersion that is put through dialysis for 2 weeks to remove any remaining metal. This $2 \%$ aqueous dispersion is used to prepare all of the subsequent dispersions for deposition.

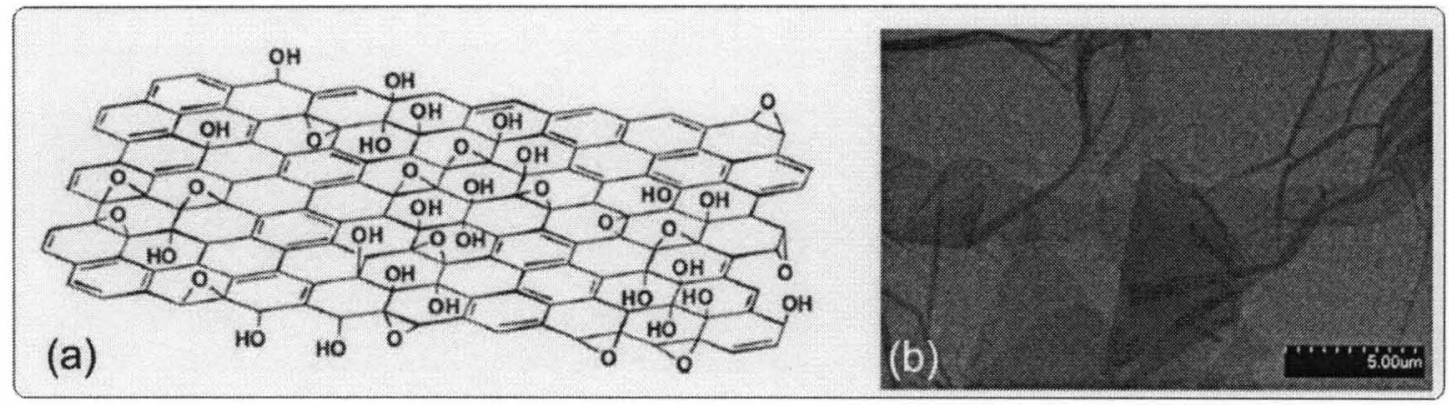

Figure 3.6 (a) Schematic of a proposed structure of graphene oxide. (b) Graphene oxide film dispersed on $\mathrm{SiO}_{2} / \mathrm{Si}$ substrate.(adapted from [59]).

\subsection{Reduced Graphene Oxide (rGO) Synthesis Techniques}

The reduction of oxygen content in the oxidized graphene (GO), results in reduced graphene oxide (rGO). Two methods are mainly followed to reduce GO: the thermal 
curing of the GO sample and the chemical treatment of GO. Prof. Walt A. de Heer's research group showed that thermal curing of graphene oxide at $180{ }^{\circ} \mathrm{C}$ for 16 hours reduces the GO to measurable levels [21]. Prof. Ajayan's research group followed a two step chemical reduction process, deoxygenation with $\mathrm{NaBH}_{4}$ followed by dehydration with concentrated $\mathrm{H}_{2} \mathrm{SO}_{4}$ to restore the graphene structure for $\mathrm{GO}$ [62]. Hydrazine treatment of GO is the widely followed reduction process. Figure 3.7 shows TEM images of hydrazine reduced single sheet of rGO [63].

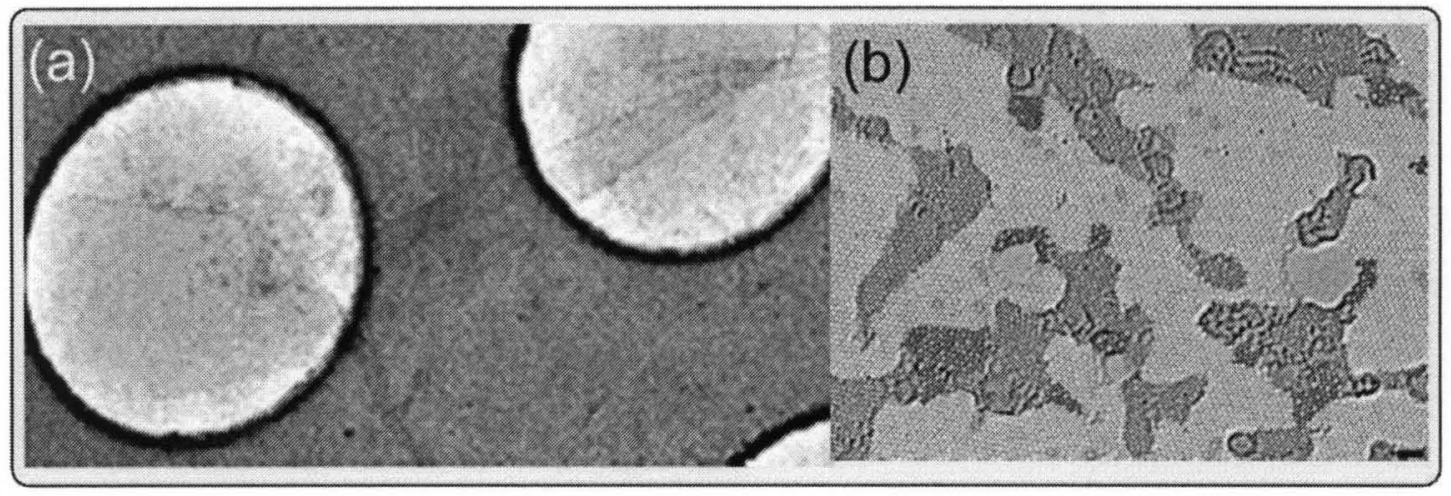

Figure 3.7 (a) TEM image of a partly folded rGO sheet. (b) Atomic resolution TEM image of single layer rGO sample with color added to highlight the different features.(adapted from [63])

The TEM images of the hydrazine reduced GO reveal that the reduced graphene oxide sheet is comprised of defect free crystalline graphene areas (light gray) interspersed with oxidized graphene areas (dark gray) and topological defects (blue, green and yellow). The crystalline graphene areas are $3-6 \mathrm{~nm}$ in size covering $\sim 60 \%$ of the surface, while the oxidized graphene areas are $1-2 \mathrm{~nm}$ in size and covering $\sim 30 \%$ of the surface. Topological defects are further divided into isolated defects (pentagons, heptagons, green and holes, yellow formation while reduction) and extended defects (quasi-amorphous $\mathrm{sp}^{2}$ single layer carbon structures, blue). 


\subsection{Characterization Measurements}

This section provides a summary of general characterization measurements results obtained for graphene, GO and rGO samples (samples prepared using varied synthesis procedures). The main focus of this summary is to present a better insight towards the electronic band structure of these samples using universal characterization techniques.

\subsubsection{Electrical Measurements}

Electrical measurements including current-voltage (I-V) curves, gate dependence measurements and temperature dependence measurements of I-V or gate dependence curves provide an idea regarding the characteristic behavior of the material (such as metallic, semiconducting or insulating). The nature of the I-V curve, i.e. linear or nonlinear, provides information regarding the distribution of energy states near the Fermi energy. Gate dependence measurements determine what influence a shift in the Fermi energy has (with respect to valence and conduction band edge) on the conductance of the material. The temperature dependence of the resistance or conductance provides information on the electrical characteristics of the material. A decrease in resistance with decreasing temperature indicates metallic behavior, while an increase in resistance with decreasing temperature is a signature for semi-conducting behavior. Figure 3.8 shows the results of electrical measurements performed on monolayer and few layer graphene samples. 


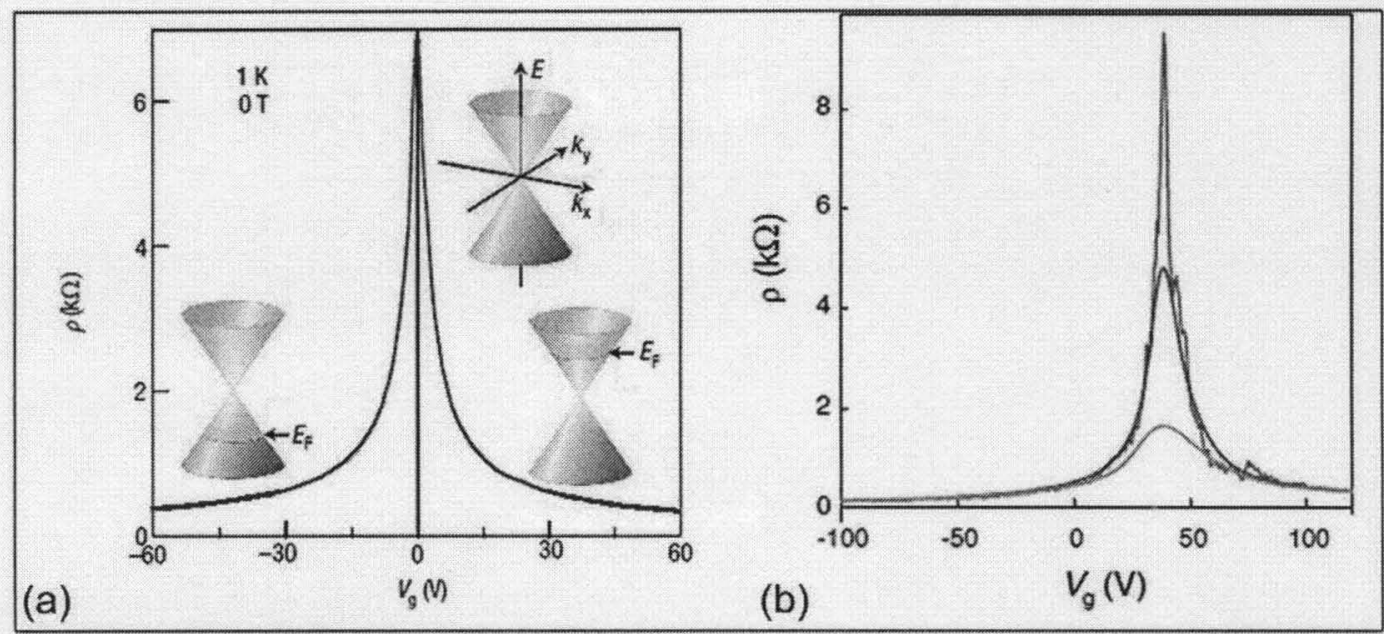

Figure 3.8 (a) Ambipolar electric field effect in monolayer graphene at $1 \mathrm{~K}$. The inset shows the changes in the position of the Fermi energy $E_{F}$ with changing gate voltage $\mathrm{Vg}$. (b) Dependence of few layer graphene's resistivity $\rho$, on gate voltage for different temperatures ( $\mathrm{T}=5,70$ and $300 \mathrm{~K}$ from top to bottom curves). (adapted from $[8,59]$ )

The devices with the back gate geometry showed semi-metallic (zero bandgap) ambipolar behavior with non-zero conductivity for all the gate voltages. The minimum conductance point (Dirac point) was observed at non-zero gate voltage at room temp in atmosphere.

Electrical measurements from dual gate configuration for mono- and bi-layer graphene samples are as shown in Figure 3.9. In this configuration, the bi-layer graphene is supported on a heavily doped $\mathrm{Si} / \mathrm{SiO}_{2}$ wafer (back gate), and a metal top-gate is fabricated using a $\mathrm{NFC} / \mathrm{HfO}_{2}$ gate insulator. Top gate is swept from $-2 \mathrm{~V}$ to $6 \mathrm{~V}$ while the back gate is swept from $-120 \mathrm{~V}$ to $80 \mathrm{~V}$. The current-voltage (I-V) characteristics for the dual gate bi-layer device show linear change. At low temperatures the $\mathrm{I}_{\mathrm{on}} / \mathrm{I}_{\mathrm{off}}$ for the bilayer graphene can be increased to $\sim 2000$. 


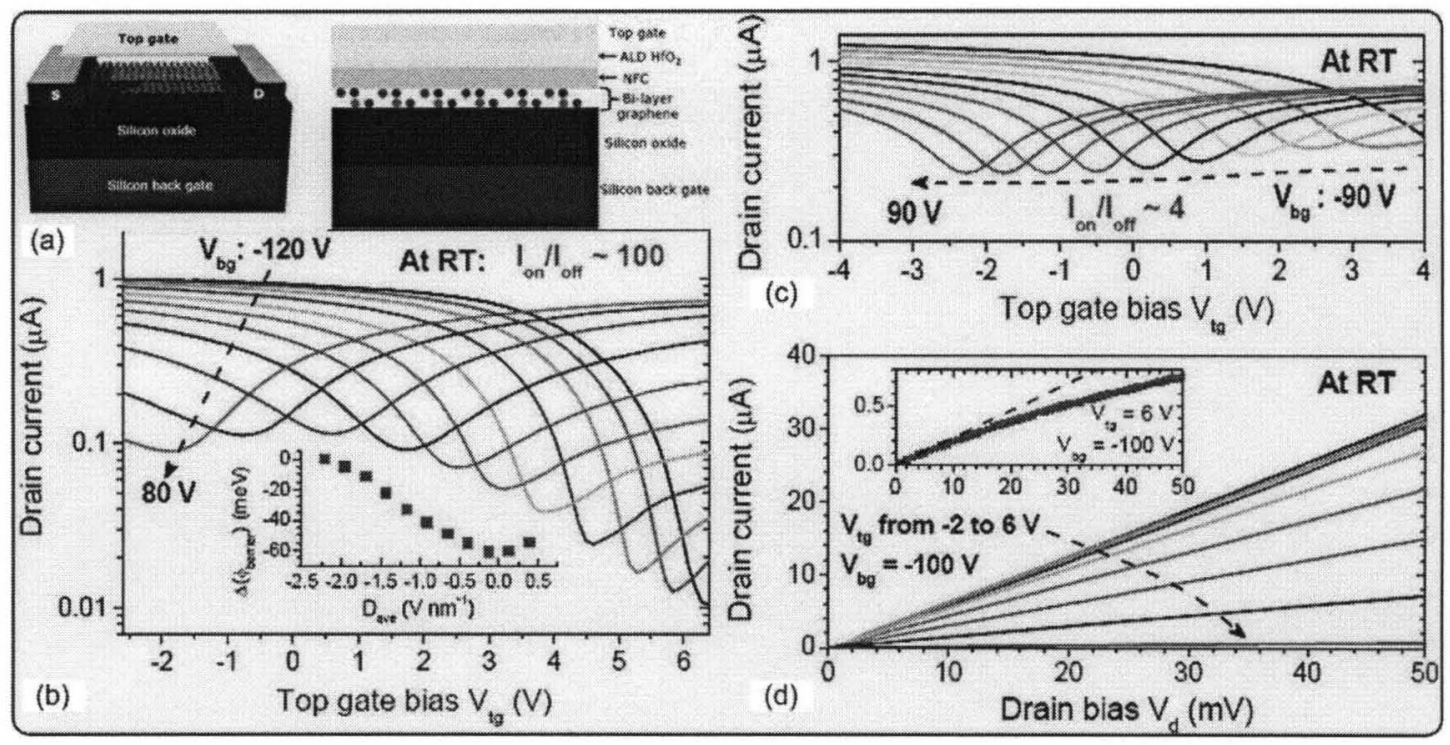

Figure 3.9 (a) Schematic of dual gate configuration with layer by layer description. (b) Transfer characteristics of dual gated bi-layer graphene device at room temperature. (c) Transfer characteristics of dual gated monolayer graphene device. (d) I-V characteristics of bi-layer graphene sample with back gate at $-100 \mathrm{~V}$ and top gate being swept from -2 volts to 6 volts.(adapted from [13]).

The asymmetry in the bandstructure of the bi-layer graphene induced from the perpendicular field (dual gate geometry) is due to the strong coupling between the atoms lying directly over each other in the two layers. The upper valence and lower conduction bands move apart to form a direct band gap that is slightly offset from the K-points as shown in Figure 3.10 [22]. The gap size is determined by the sum of the resulting dielectric displacement fields produced by the back and top-gates, $D_{\mathrm{b}}+D_{\mathrm{t}}$, while the Fermi level depends on the difference, $D_{\mathrm{b}}-D_{\mathrm{t}}$. At the neutrality point, $D_{\mathrm{b}}=D_{\mathrm{t}}$, the current on/off ratio at room temperature achieved by the dual-gated bi-layer device is $\sim 100$ and that for monolayer device is $\sim 4$. The induced band gap of this device is fieldtunable with a maximum value of $130 \mathrm{meV}$. 

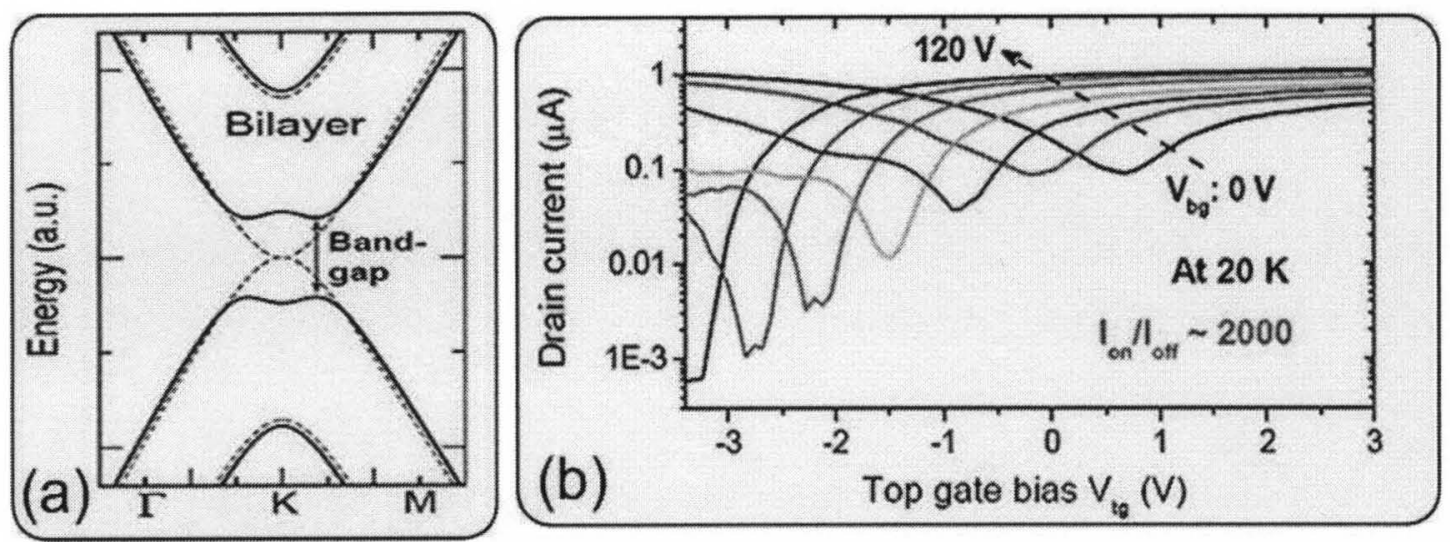

Figure 3.10 (a) Schematic illustrating the bandstructure changes induced in bilayer graphene by an applied perpendicular electric field. The dotted curves represent the bandstructure in the absence of the field, while the solid line shows the bandstructure in the presence of a strong field. (b) Temperature dependence of bi-layer graphene device. (adapted from [22])

Another variation in the structure of grapheme devices for transport measurements is graphene nanoribbon (GNR) devices. Two approaches, e-beam lithography patterning and chemical treatment, were followed to achieve width dimensions as small as $5 \mathrm{~nm}$. In the e-beam lithography method followed by IBM group [64], large graphene sheets $(\sim 20 \mu \mathrm{m})$ are patterned, using negative e-beam resist hydrogen silsesquioxane (HSQ), to form nanoribbons of widths varying from 10-100nm and 1-2 $\mu \mathrm{m}$ in length [16]. Figure 3.11 shows conductance measurements on three of these devices [14].
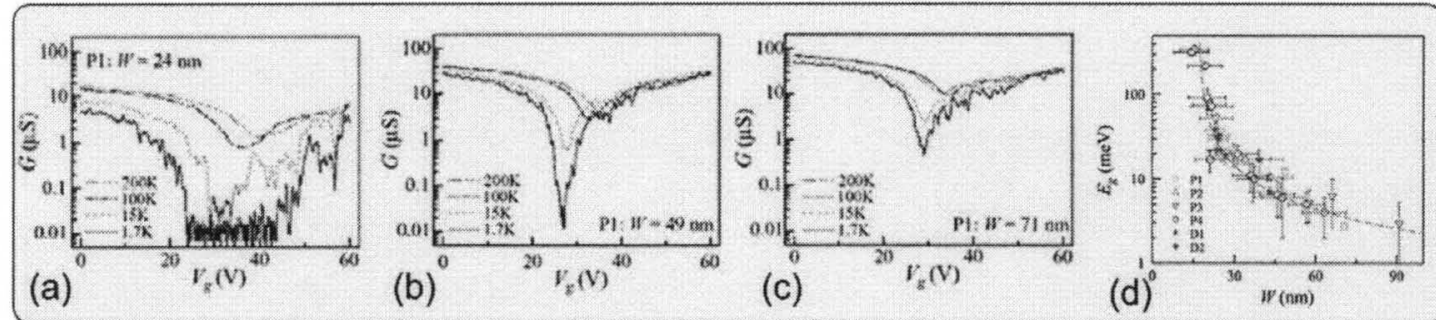

Figure 3.11 (a)-(c) Conductance measurements of GNR devices as function of gate voltage measured at different temperatures. (d) Plot of energy gap versus width of GNR. (adapted from [14]). 
Energy band gap variation with the GNR width is also presented in Figure 3.11. A band gap of $0.2 \mathrm{eV}(200 \mathrm{meV})$ was observed from a ribbon of width $15 \mathrm{~nm}$.

Using the chemical method GNRs with widths under $10 \mathrm{~nm}$ have been obtained. Figure 3.12 summarizes the GNR formation process while Figure 3.13 summarizes the various results obtained from these GNRS. The energy gap from the narrowest ribbons $(W=2 \mathrm{~nm})$ was estimated to be $0.4 \mathrm{eV}$ and $\mathrm{I}_{\mathrm{on}} / \mathrm{I}_{\text {off }}$ ratios of up to $10^{7}$ were obtained.

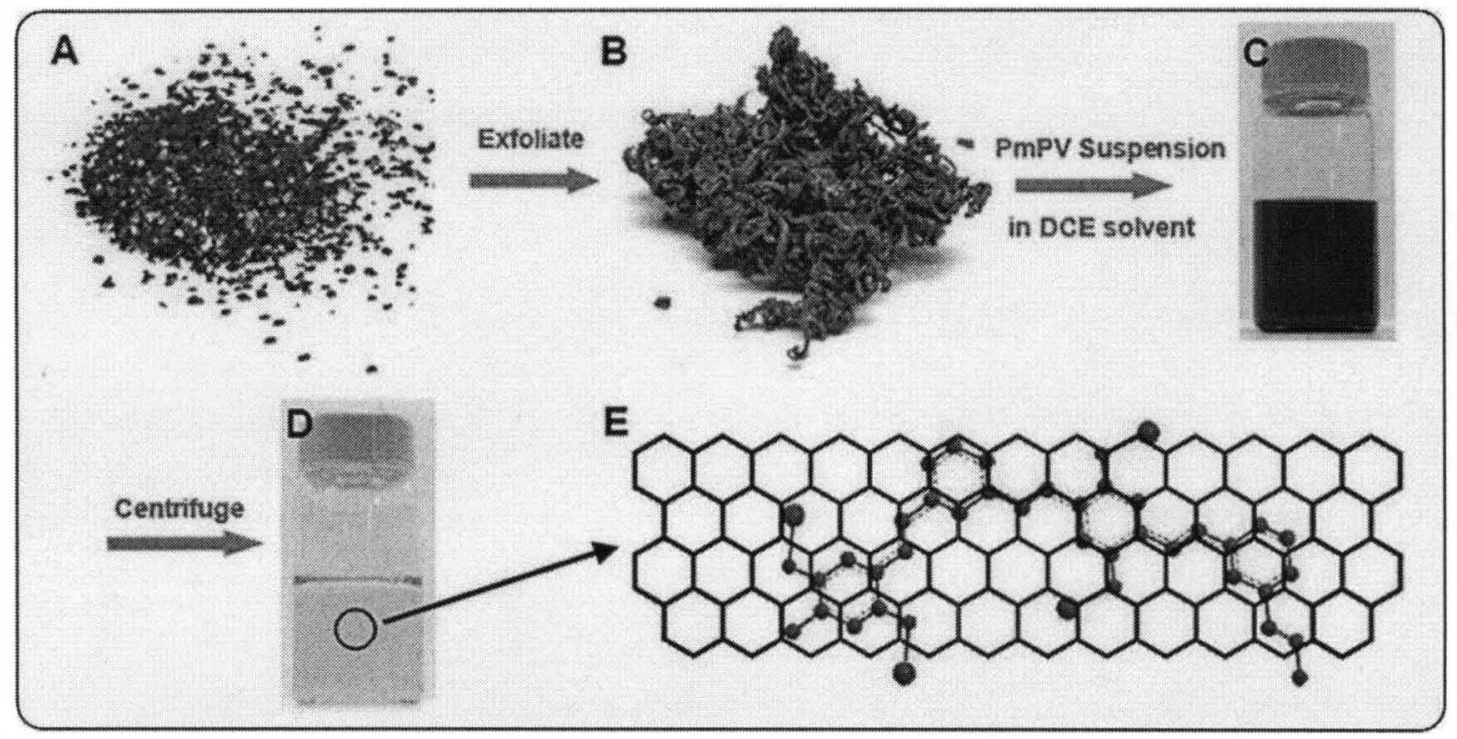

Figure 3.12 Schematic process of making graphene nanoribbons suspensions. (adapted from [16]).

The electrical measurements on graphene oxide (GO) demonstrated its insulating behavior; however, after a suitable reduction step it can be made to be conducting. The main approaches for reduction are thermal reduction and hydrazine $\left(\mathrm{N}_{2} \mathrm{H}_{4}\right)$ treatment. Figure 3.14 summarizes the result after thermal reduction of GO [65]. The improvement in electrical conductivity of GO upon reduction is attributed to the reduction in the oxidation content. The current-voltage (I-V) curves show increase in linearity with increasing reduction and an overall decrease in resistance. 


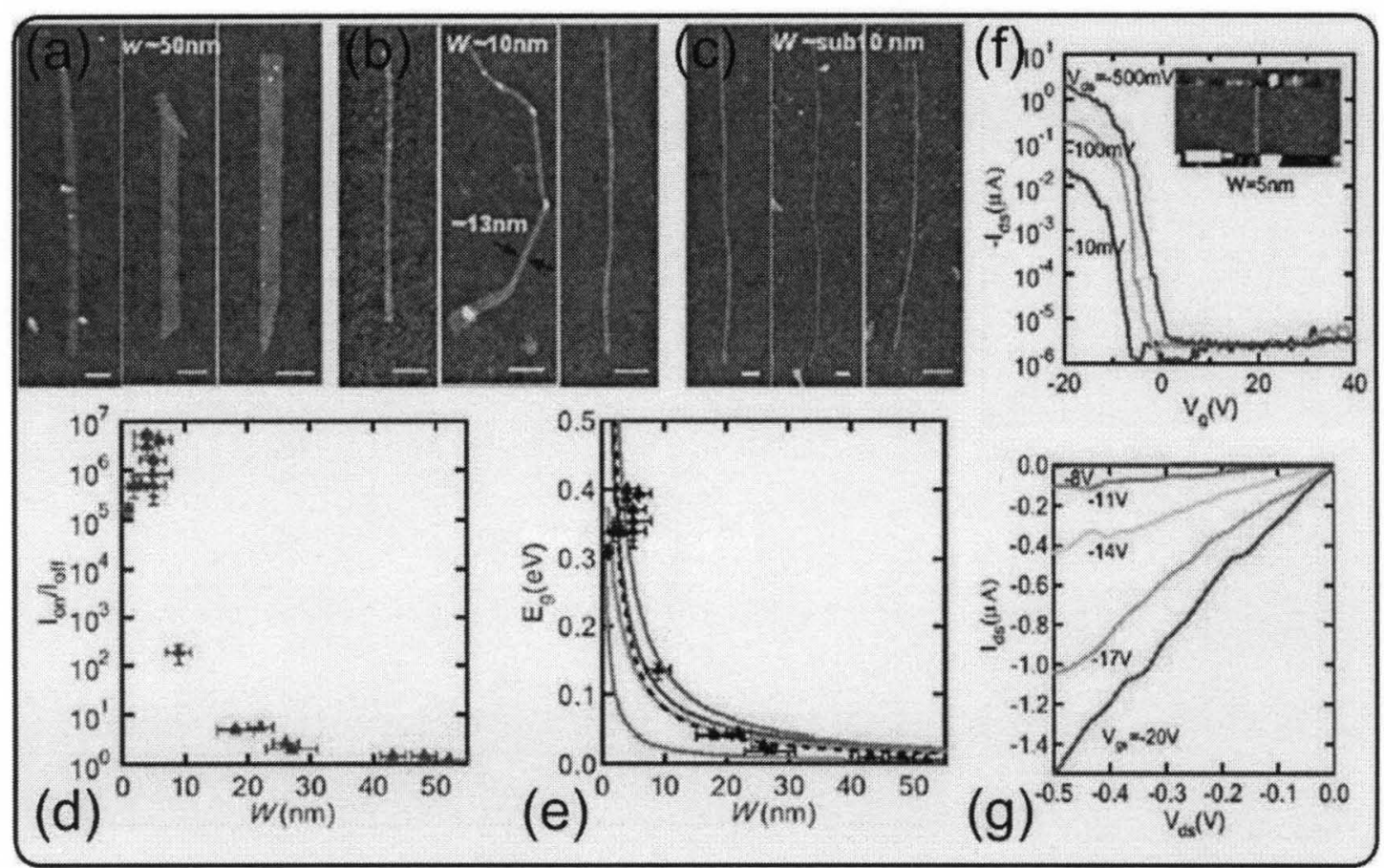

Figure 3.13 (a) -(c) AFM images of GNR. (d) Plot of $\mathrm{I}_{\mathrm{on}} / \mathrm{I}_{\text {off }}$ ratio versus the GNR width. (e) Plot of energy gap versus the GNR width. (f) Gate dependence measurements for a $5 \mathrm{~nm}$ wide GNR. (g) I-V characteristics for the sample in (f). (adapted from [16])

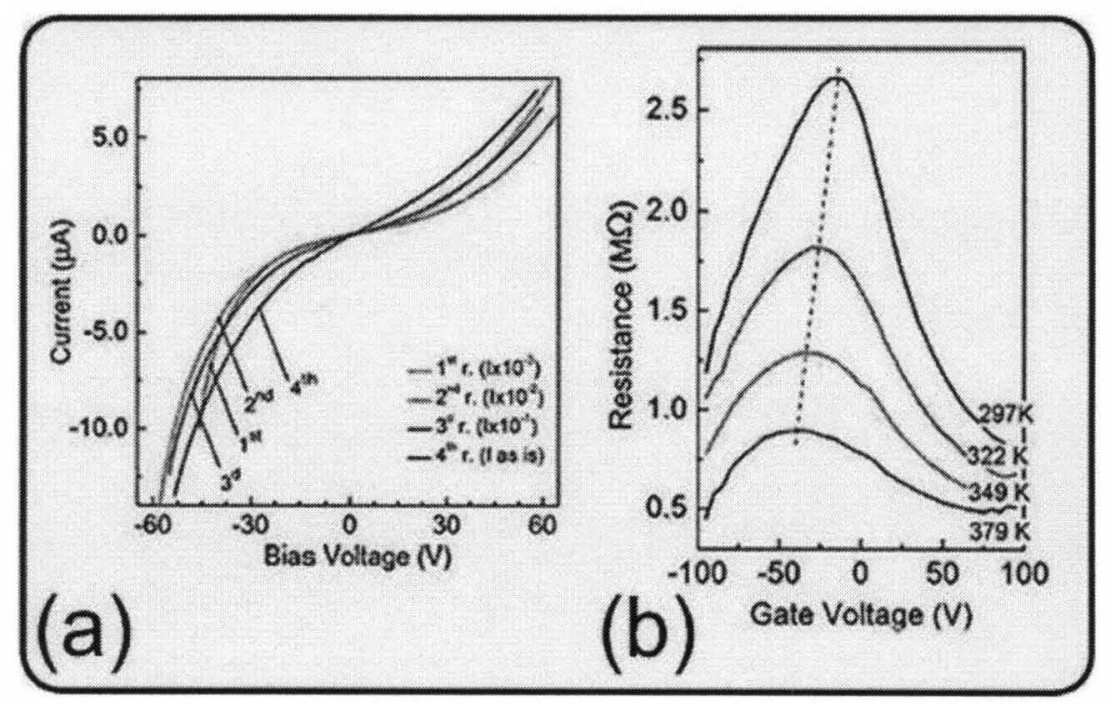

Figure 3.14 (a) I-V curve for different reduction steps. (b) Change in resistance with respect to gate voltage at different temperatures. Increasing temperature increase the reduction which in turn reduces the resistance and increases the linearity of I-V curve. (adapted from [65]). 
The other approach to reduce GO is by treating it with hydrazine. The measurement results for hydrazine treated samples are shown in Figure 3.15 [66]. Again, the linearity in the I-V increases with an increase in reduction duration. Resistance with respect to gate voltage also decreases with an increase in reduction (increase in temperature). In Figure 3.15 (b), one curve presents the effect of 5 second hydrogen $\left(\mathrm{H}_{2}\right)$ plasma on the GO sample. Treatment with $\mathrm{H}_{2}$ plasma reduces the $\mathrm{GO}$ at much faster rate.
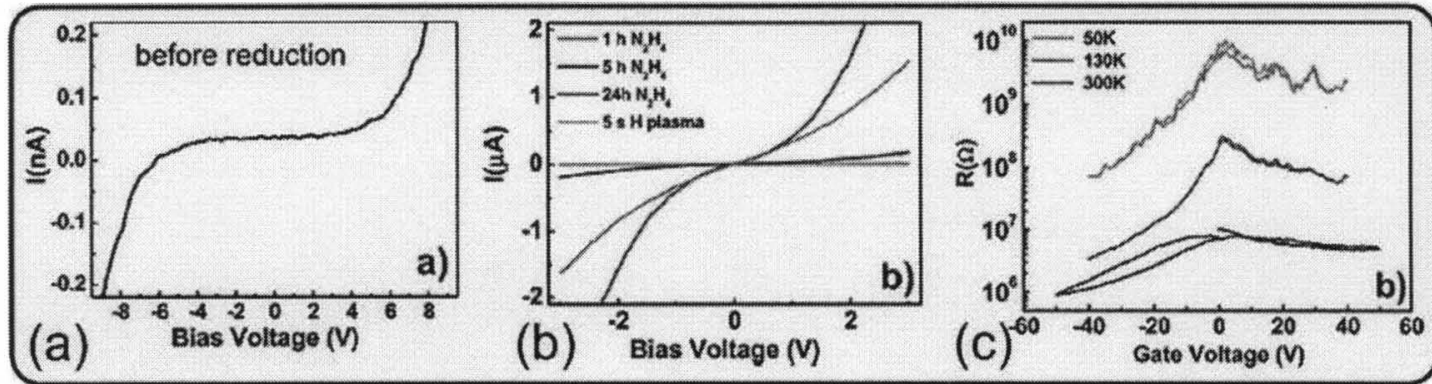

Figure 3.15 (a) I-V curve for GO (before reduction). (b) I-V curve for 4 different steps of reduction. (c) Change in resistance with respect to gate voltage at different temperatures. (adapted from [66]).

\subsubsection{Optical Measurements}

Optical measurement techniques, such as absorption spectroscopy and photoluminescence (PL) spectroscopy, provide information regarding the band structure of the material. Features in the wavelength spectrum of the absorption measurements will provide the energy difference between the valance and conduction band. The feature in the PL data represents the transition from excited energy levels to lower energy levels (assuming the levels are optically active). Figure 3.16 summarizes the absorption spectra of graphene, GO and rGO observed by various groups. Monolayer graphene shows a constant $\sim 97 \%-98 \%$ transmittance in the visible range $(400 \mathrm{~nm}-700 \mathrm{~nm})$ [23]. With the 
increase in the number of layers the transmittance decrease by $2.3 \%$ per layer (\% layers shows $\sim 88 \%$ transmittance), as shown in Figure 3.16 (a). In the ultra-violet (UV) region of the absorption spectra, monolayer graphene shows a strong peak near $260 \mathrm{~nm}-265$ $\mathrm{nm}(4.68 \mathrm{eV}-4.7 \mathrm{eV})$ as shown in Figure 3.16 (b) [24]. In the UV region of the absorption spectra, GO shows a strong peak around $233 \mathrm{~nm}$ with a small shoulder near $320 \mathrm{~nm}$ [25-27]. The absorption peak in the GO drops off to zero absorption near 450$500 \mathrm{~nm}$ and stays flat for the rest of the visible and near infra-red (NIR) spectrum. In case of $\mathrm{rGO}$, a strong peak (similar to graphene) is observed in the UV spectra (near 260nm) which fall off slowly to near zero absorption value. The visible and NIR spectra for rGO stays flat and resembles a more like graphene spectra.

The strong feature observed in the UV absorption spectra for all the samples is attributed to the $\pi-\pi^{*}$ transitions of $\mathrm{sp}^{2}$ hybridized $\mathrm{C}=\mathrm{C}$ (double bonds). The shoulder at $320 \mathrm{~nm}$ seen for GO is attributed to the n-nonbonding to $\pi^{*}$ transitions due to the presence of $\mathrm{C}=\mathrm{O}$. The shift in the peak location for graphene, $\mathrm{GO}$ and $\mathrm{rGO}$ indicates to the amount of decoupling of the $\pi$ electrons in the pi bonding system (more the decoupling less the energy difference between the $\pi / \pi^{*}$ orbitals). The presence of oxygen bonds in the GO reduces the extent of the decoupling which in turn pushes the $\pi / \pi^{*}$ orbitals slightly apart requiring higher energy (lower wavelength) transition. 

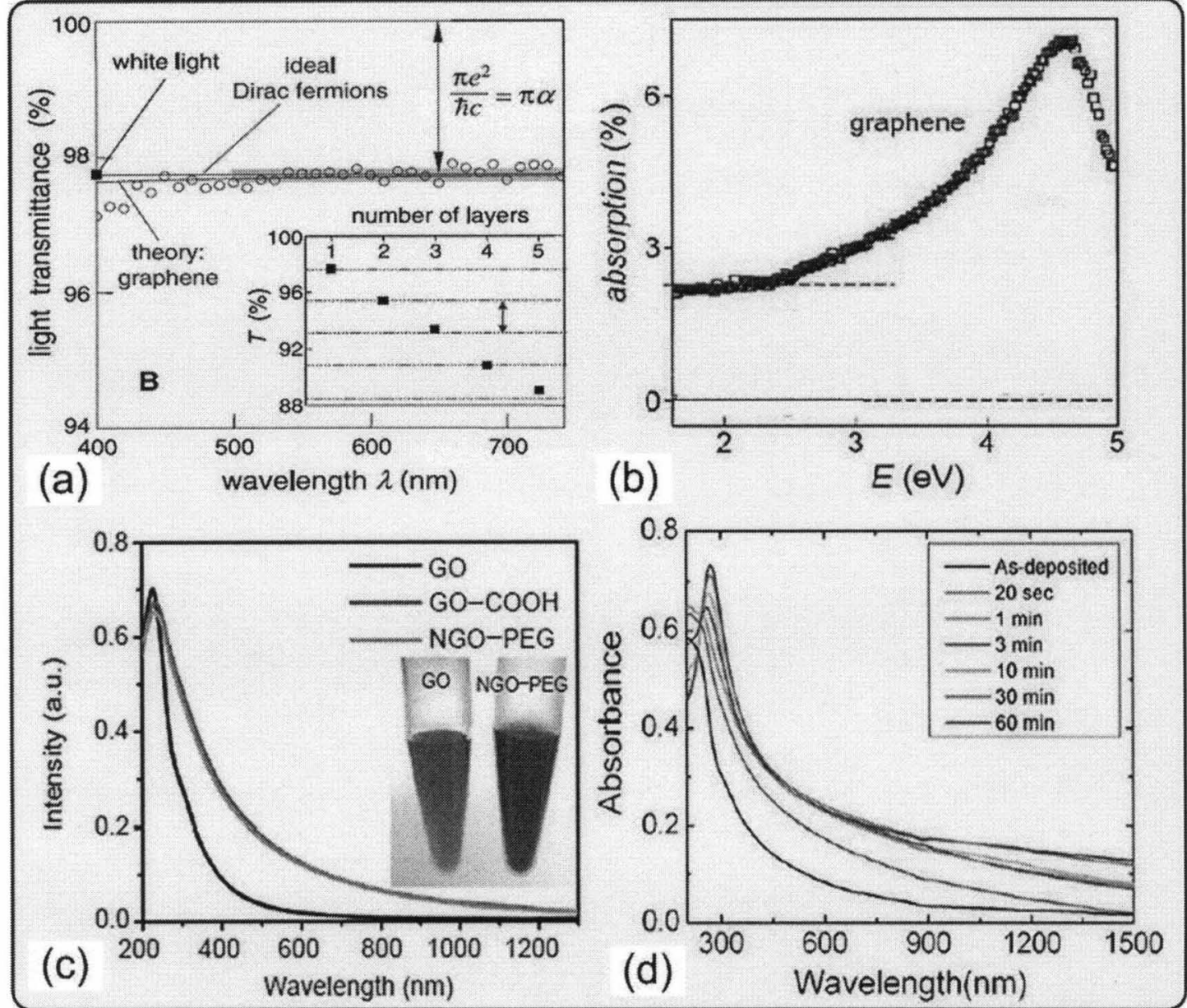

Figure 3.16 (a) I-V curve for GO (before reduction). (b) I-V curve for 4 different steps of reduction. (c) Change in resistance with respect to gate voltage at different temperatures. (adapted from [23-27]).

Similar features in the GO, rGO and graphene absorption spectra have been reported by a number of groups; however, for PL measurements, there are large differences in the reported spectra. Widely different peak energies for the PL in GO have been reported. The PL measurements on GO and rGO are summarized in Figure 3.17. 


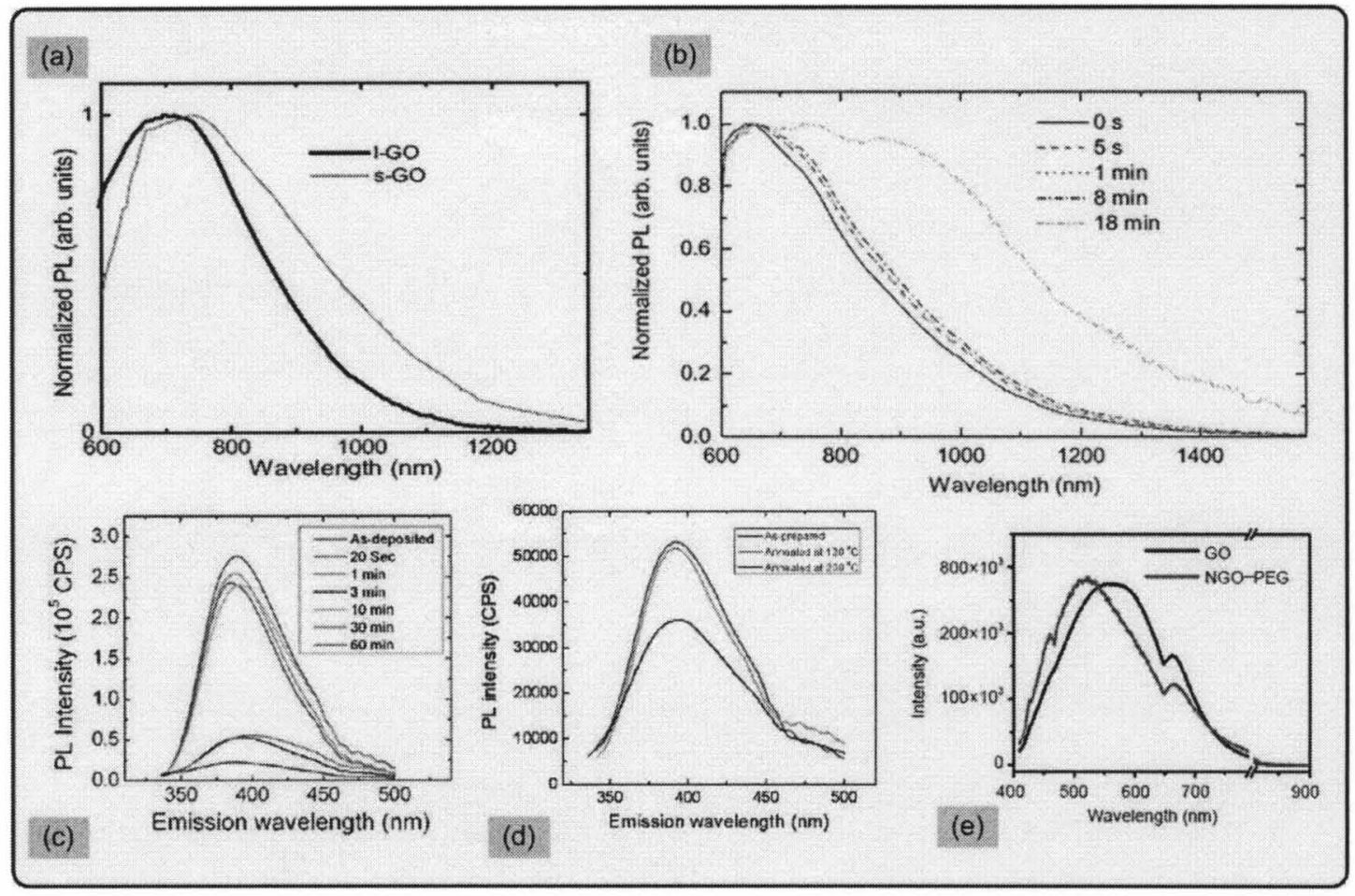

Figure 3.17 (a)-(b) Adapted from Luo et al. [28], shows PL at $\sim 750 \mathrm{~nm}$. A small red shift in PL location with increasing reduction is shown in (b). (c)-(d) Adapted from Eda et al. [25], PL at $\sim 390 \mathrm{~nm}$ is as shown. (c) Shows the change in PL intensity with increasing hydrazine reduction time. (d) Represents the change in PL intensity with reduction through annealing at different temperature. (e) Adapted from Sun et al. [27], shows GO PL at $\sim 570 \mathrm{~nm}$.

Luo et al. observe a broad photoluminescence peak centered at $1.65 \mathrm{eV}(750 \mathrm{~nm})$ which shifts to somewhat lower energy following reduction [28]. Nanometer scale strips of GO (measured by Sun et al.) show somewhat higher energy luminescence $(2.18 \mathrm{eV}$, $570 \mathrm{~nm}$ ) possibly because of quantum confinement [27]. Finally, measurements of GO and partially reduced GO (rGO) by Eda et al. show much higher energy luminescence centered at $3.2 \mathrm{eV}(390 \mathrm{~nm})$ [25]. Thus, the GO optical spectrum clearly varies with the synthesis conditions and geometry. No PL measurements were reported for graphene samples, confirming graphene's zero band gap band structure. 


\subsubsection{Raman processes in Graphene}

A generalized Raman spectrum showing different features in graphene is as shown in Figure 3.18. An understanding of the processes responsible for the different peaks provides information which in turn helps in categorizing graphene in many ways. Their shape and amplitude ratio determine the quality and number of layers present in the graphene sample. The Raman processes in graphene are divided into two main categories one- phonon resonance Raman scattering modes and two-phonon resonance Raman scattering modes. These one-phonon resonance modes are further divided into first order (or single resonance) and second order (or double resonance) processes, while the twophonon resonance modes can exist in second order (double resonance) and third order (triple resonance) Raman scattering processes.

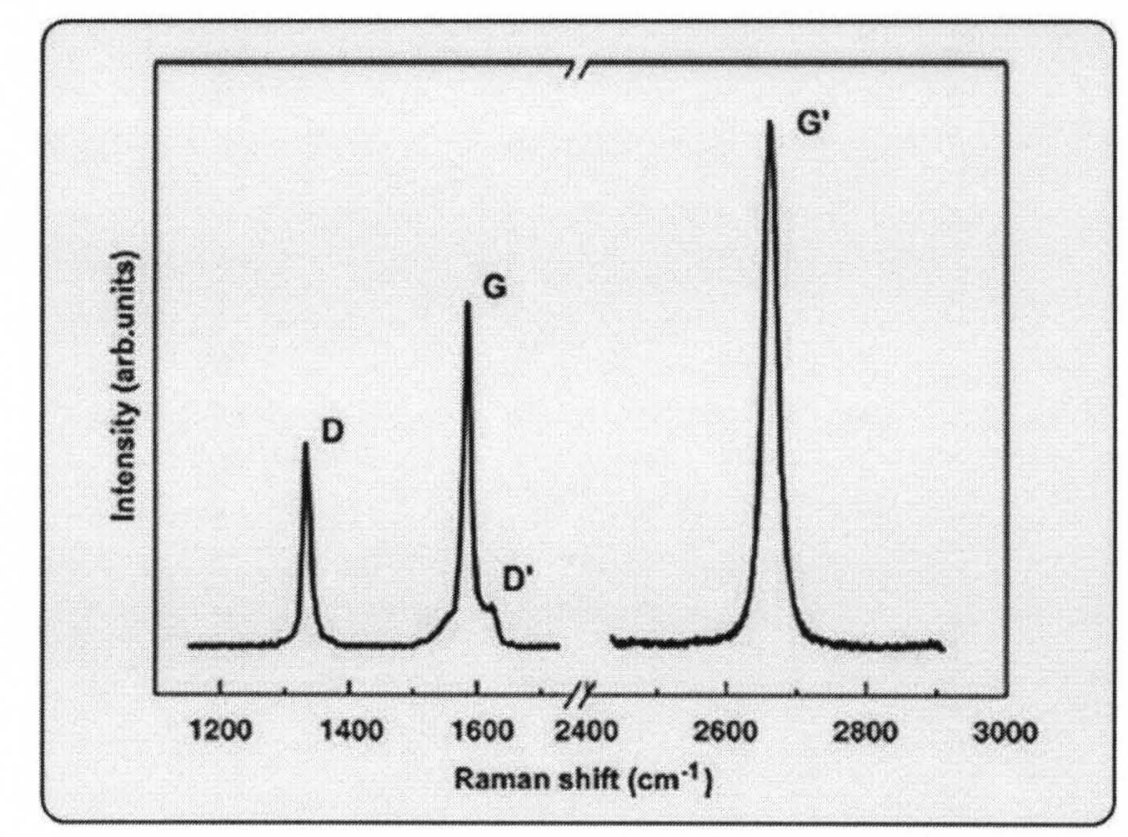

Figure 3.18 Schematic of the generalized Raman spectrum of graphene. (adapted from [45]. 
The G-band, appearing at $\sim 1582 \mathrm{~cm}^{-1}$, is the only band coming from a normal first order Raman scattering process in graphene. The $\mathrm{G}$ band is Raman active for all $\mathrm{sp}^{2}$ carbon structure due to the presence of doubly degenerate (iTO and LO) modes at the Brillouin zone center $(\Gamma)$. This band corresponds to the tangential mode in-plane stretching and bending of $\mathrm{C}-\mathrm{C}$ bonds in the hexagonal planar structure (i.e. the relative movement of sublattice A and B against each other) as shown in Figure 3.19. The G band is non dispersive in nature (its Raman shift does not change with incident photon energy).

The second-order (double resonance, DR) Raman processes for $\mathrm{sp}^{2}$ carbon material can be explained as (1) the electron absorbs a photon at $\mathrm{k}$ state (2) scatters to $\mathrm{k}+\mathrm{q}$ state (3) scatters back to a k state and (4) emits a photon by recombining with the hole at a k state (Figure 3.20) [67]. Therefore, in DR Raman process, two resonance conditions for three intermediate states should be satisfied, in which the intermediate $\mathrm{k}+\mathrm{q}$ is always a real electronic state and either the initial or the final $\mathrm{k}$ state is a real electronic state.

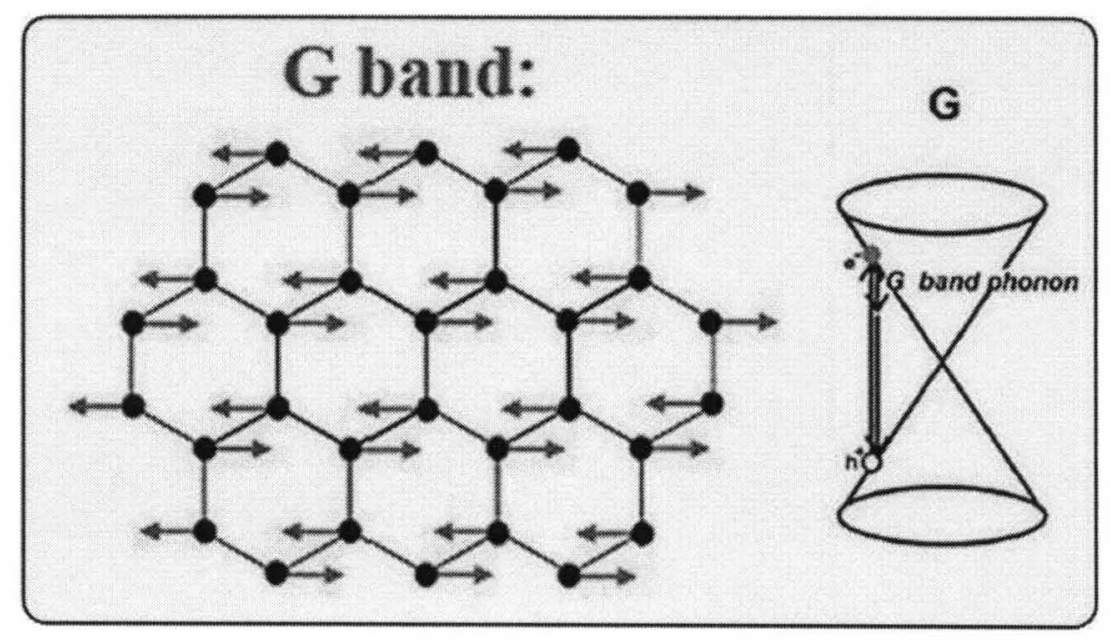

Figure 3.19 Schematic of one-phonon first order $\mathrm{G}$ band process. (adapted from $[45,47]$ ) 


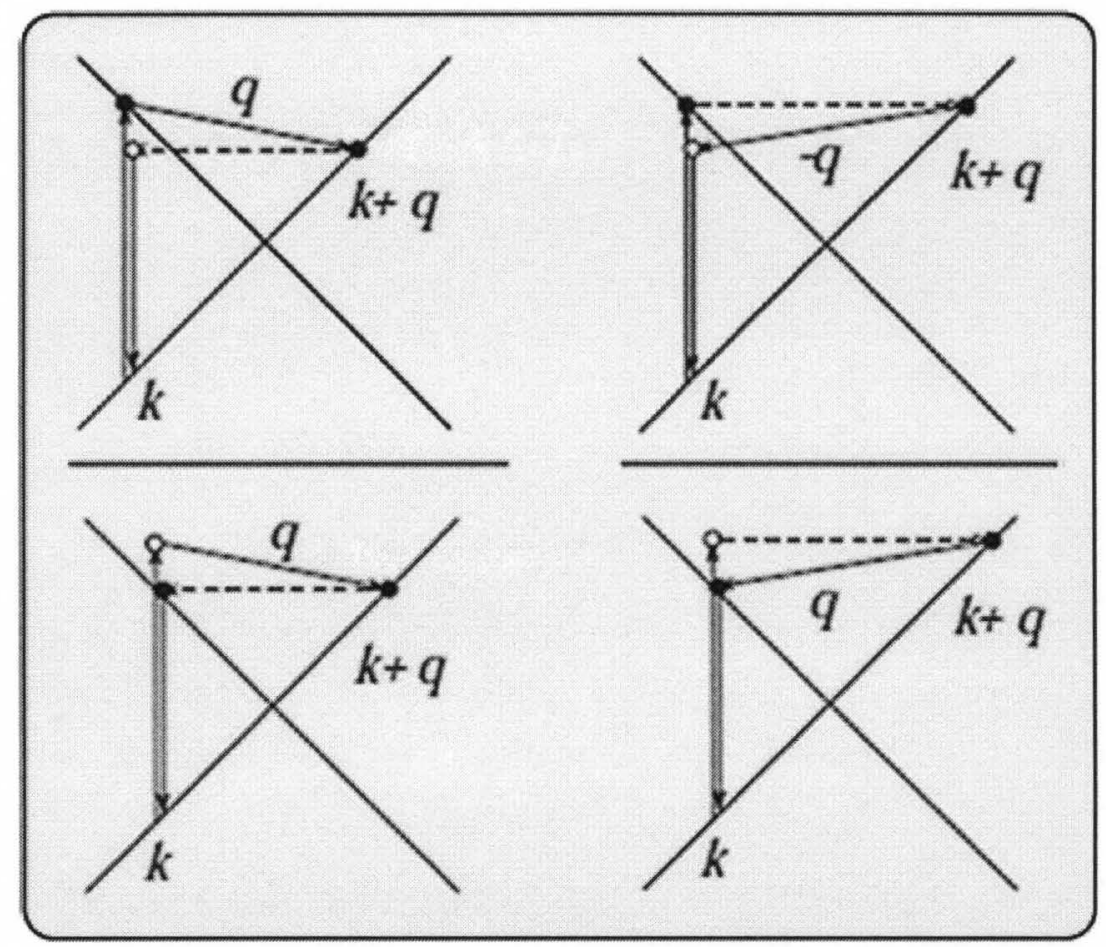

Figure 3.20 Schematic of second order (double resonance, DR) process. (adapted from [65]).

The disorder induced one-phonon second order (double resonance) giving rise to D band, appearing at $\sim 1320 \mathrm{~cm}^{-1}$, involves both an iTO phonon and a crystal defect. In this DR process, the electron absorbed at $\mathrm{k}$ state at the $\mathrm{K}$ point is elastically scattered by a defect to $\mathrm{k}+\mathrm{q}$ state near the $\mathrm{K}$ ' point. This electron then scatters back to the $\mathrm{k}$ state near the $\mathrm{K}$ point involving an inelastic scattering event with iTO phonon and emits a photon by recombining with the hole at a $\mathrm{k}$ state near $\mathrm{K}$ point. $\mathrm{K}$ and $\mathrm{K}$ ' point are inequivalent in the sense that they each corresponds to a separate sub-lattice (carbon atoms A and B), and since the $\mathrm{D}$ band involves both the $\mathrm{K}$ and $\mathrm{K}$ ' points, the process is called an inter-valley process. An increase in energy of the incident photon relative to the Dirac point moves the resonance $\mathrm{k}$ state vector away from the $\mathrm{K}$ point, which increases the corresponding $\mathrm{q}$ 
vector for the phonon, as measured from the $\mathrm{K}$ point. Thus by changing the laser energy, the dispersive nature of the $\mathrm{D}$ band can be observed.

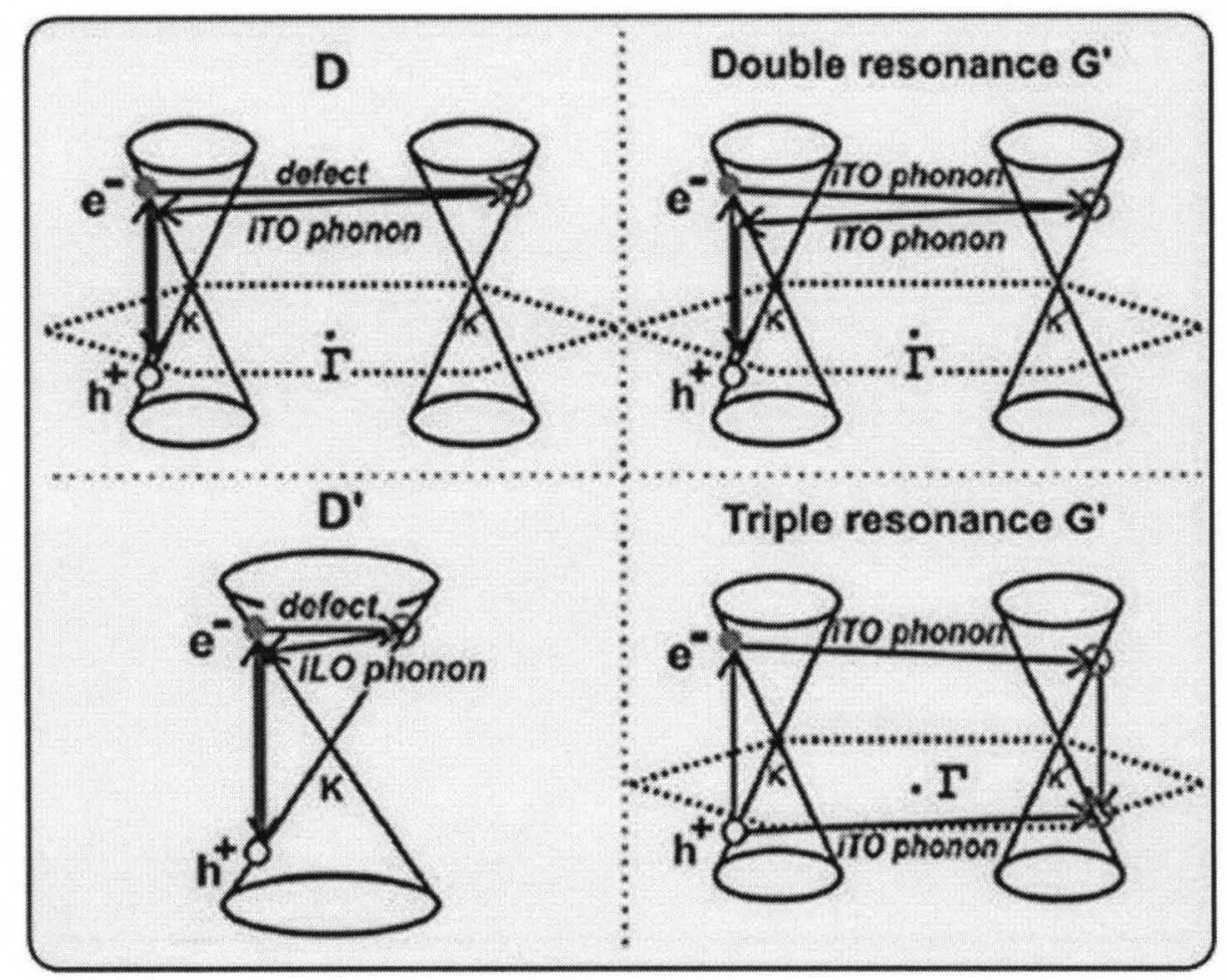

Figure 3.21 Schematic of second order (double resonance, DR) Raman process. D, D' and G' processes are illustrated. (adapted from [45]).

The G' band (or 2D band), appearing at $\sim 2640 \mathrm{~cm}^{-1}$, involves two iTO phonons. This band again is an inter-valley process. Here, both the scattering events required for the DR process are due to inelastic scattering of iTO phonons. The electron absorbed at k state in $\mathrm{K}$ point is inelastically scattered by an iTO phonon to $\mathrm{k}+\mathrm{q}$ state near the K' point. This electron then scatters back to the $\mathrm{k}$ state near the $\mathrm{K}$ point involving one more inelastic scattering event with iTO phonon and emits a photon by recombining with the hole at a $\mathrm{k}$ state. Since two iTO phonons are involved, the G' band frequency is double the frequency of $\mathrm{D}$ band, therefore it is also called a 2D band. This process also displays dispersive nature depending on the energy of the incident light. Since the Raman process 
can also occur by scattering of the holes, and in the case of graphene where the valence and conduction bands are almost mirror bands of one another relative to the Fermi energy, a new effect, the triple resonance (TR) Raman process can occur. In the TR process, the electron absorbed at $\mathrm{k}$ state in $\mathrm{K}$ point is inelastically scattered by an iTO phonon to $\mathrm{k}+\mathrm{q}$ state near the $\mathrm{K}$ ' point. This electron then recombines with a hole emitting a photon. This hole near $\mathrm{K}^{\prime}$ point scatters back to $\mathrm{k}$ state near $\mathrm{K}$ point due to an inelastic scattering event involving iTO phonon. In this case, the electron-hole generation and both the electron and hole scattering processes will be resonant resulting in a triple resonant process. The presence of intense $G$ ' band as compared to $G$ band for single layer graphene can be explained using this TR resonance condition.

The $D^{\prime}$ band, appearing at $\sim 1620 \mathrm{~cm}^{-1}$, is an intra-valley one phonon double resonance process. As for $\mathrm{D}$ band this band is again induced by the presence of defects, however, it involves iLO phonon instead of iTO phonon involved in D band. It connects energy levels all located around the same $\mathrm{K}$ or K' point and involves an iLO phonon and defect to interact with the electron similar to the processes in D band.

For bi-layer graphene, the electron dispersion diagram is modified, resulting in two valence bands $\left(\pi_{1}\right.$ and $\left.\pi_{2}\right)$ and two conduction bands $\left(\pi_{1}{ }^{*}\right.$ and $\left.\pi_{2}{ }^{*}\right)$ near the $K, K$ ' points. These four bands results in four DR process named Pij (where $\mathrm{i}$ and $\mathrm{j}$ are the conduction band from, and to which the electron is scattered, respectively). This modifies the G' band to contain four peaks and the change up-shifts the G' band location compared to single layer graphene. 


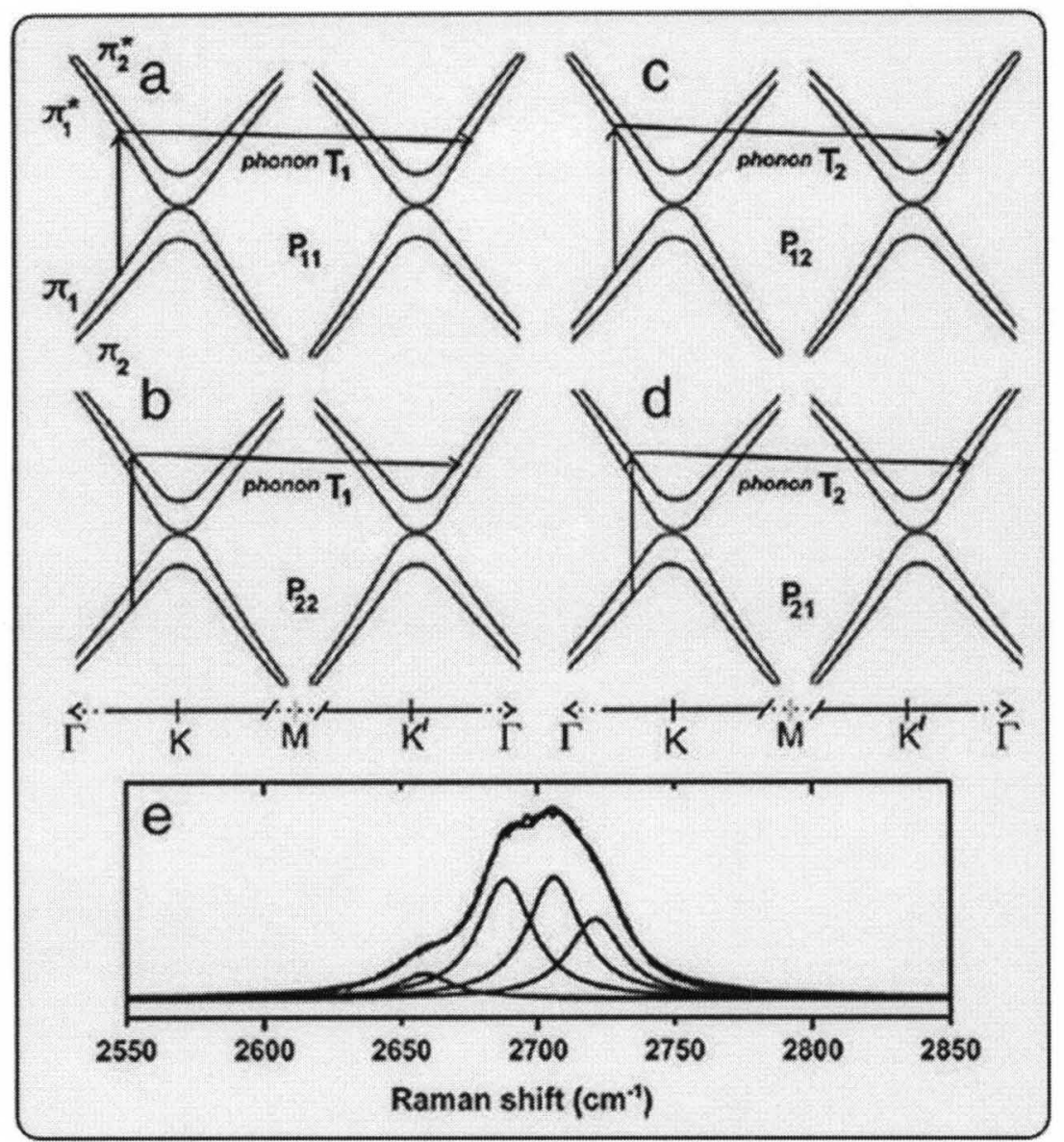

Figure 3.22 Schematic view of bi-layer graphene near K, K' points showing both $\pi_{1}$ and $\pi_{2}$ bands. The four DR processes are (a) $P_{11}$, (b) $P_{22}$, (c) $P_{12}$, (d) $P_{21}$. (e) G' band for bilayer graphene with four fitted Lorentzians are also shown. (adapted from [45]).

The appearance of the D and D' band combined with their intensity in a graphene sample Raman spectra indicates the presence of defects on the structure. The shape of G' band combined with intensity ratio of $\mathrm{G}^{\prime} / \mathrm{G}$ can be used to infer about the number of layers of graphene sheets present in the sample. Figure 3.23 summarizes the variation in Raman features seen for different samples $[67,68]$. 


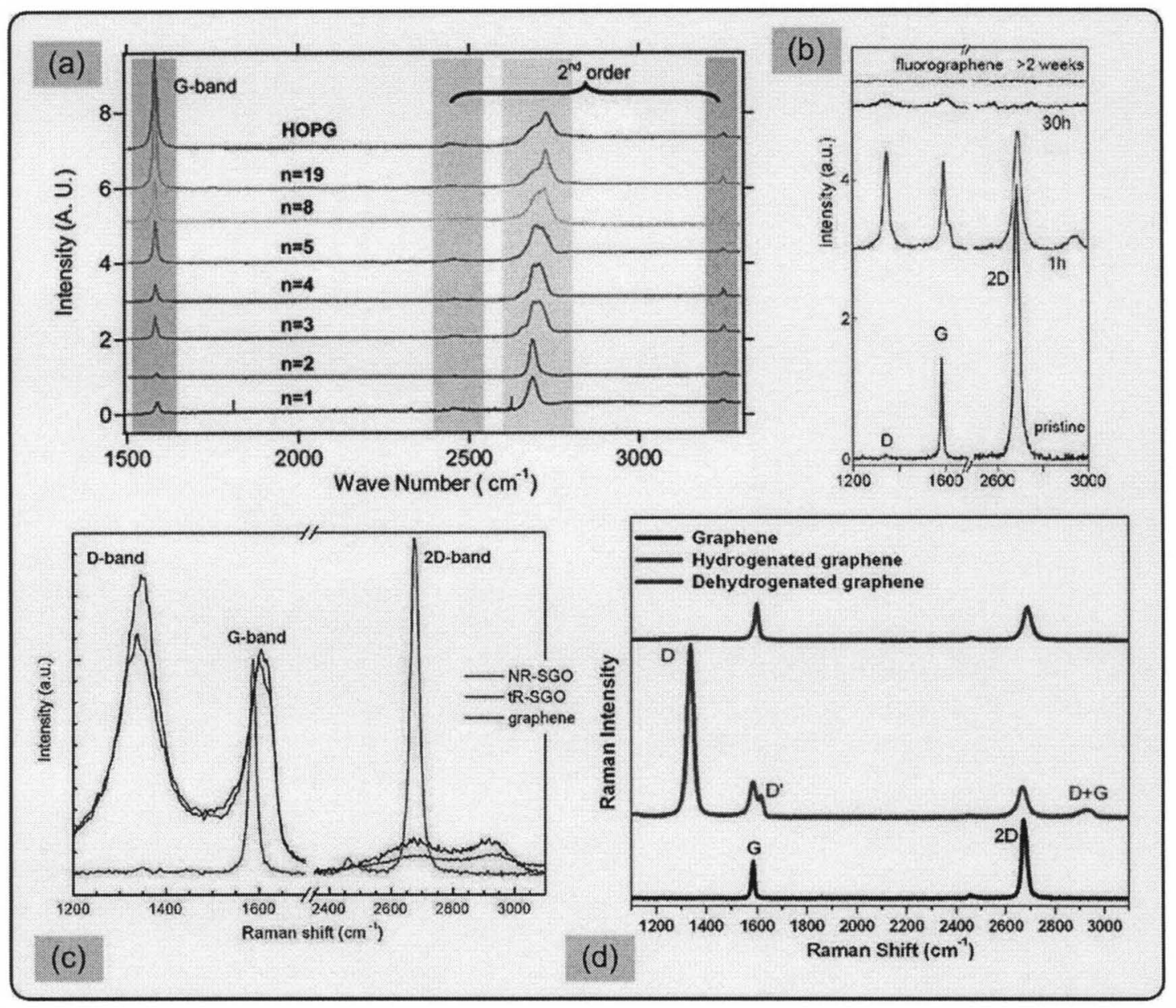

Figure 3.23 (a) Variation in Raman peaks shape and intensity with the change in the number of graphene layers. (b) Changes in Raman spectra of monolayer graphene with increasing fluorination. (c) Difference in Raman spectra for monolayer graphene, GO and rGO samples. (d) Evolution of Raman spectra of a monolayer graphene with hydrogenation and dehydrogenation. (adapted from [24, 65, 68, 69]

The intensity ratio $G^{\prime} / G$ is greater than 1 for less than 4 layers (for monolayer $G^{\prime} / G$ $\geq 2$ ). Different functionalization (oxidation, hydrogenation or fluorination) of graphene shows similar features in the Raman spectra (appearance of D and D' peak and reduction in the intensity of G' (2D) peak. The shape of the G' and appearance of some new features $D+G$ can be observed with functionalization of graphene. Due to the presence of similar features for different functionalization groups, Raman spectroscopy can be used 
only to differentiate between pristine graphene and functionalized graphene (and not for differentiating between functionalized groups).

\subsection{Discussion}

As seen in the previous sections, various synthesis techniques are employed to produce graphene, resulting in graphene samples having a variety of shapes, sizes and number of layers. Depending upon the configuration of the graphene sample its properties can change significantly. Good quality mono- or few layer graphene shows zero energy band behavior in electrical and optical measurements. A zero band gap results in finite minimum conductance which in turns limits the $I_{\text {on }} / I_{\text {off }}$ ratio to a very small value preventing graphene to be used as active element in any logic circuit. Also, the optical properties depend on the energy difference between the conduction and valence band. Keeping this in mind, several routes have been developed to induce and control an energy gap in graphene for shutting off the current. As mentioned earlier dual gate geometry for a bi-layer graphene can open a small tunable band gap upto $0.2 \mathrm{eV}$. The energy gap generated is still too small for any practical device application at room temperature. Another approach to introduce a band gap in graphene is to constrict its lateral dimensions to generate quasi-one dimensional (1D) GNRs. For narrow GNR, ID confined states are formed similar to those of a particle in a narrow, long quantum box. The gap opened is inversely proportional to the width of the GNR. Two approaches were followed, e-beam lithography patterning and chemical treatment. GNR with a constant width of 2-3 nm are required to create a significant band gap $(\geq 0.5 \mathrm{eV})$ which can allow room temperature operations. Unfortunately, currently e-beam lithography patterning can 
achieve GNR only around $\sim 10 \mathrm{~nm}$ in width. Chemical treatment method to produce GNR can create thinner GNR $(\sim 3 \mathrm{~nm}-5 \mathrm{~nm})$; however, chemical treatment has no control over ribbon width reproducibility, GNR edge roughness, GNR location on substrate. Angleresolved photoemission spectroscopy (ARPES) studies have shown that due to the interaction between substrate $(\mathrm{SiC})$ and the epitaxially grown graphene layer a small band splitting of up to $0.26 \mathrm{eV}$ can occur. This gap decreases as the number of layer grown increase and eventually approached zero for layers exceeding four in number, as shown in Figure 3.24 [12].

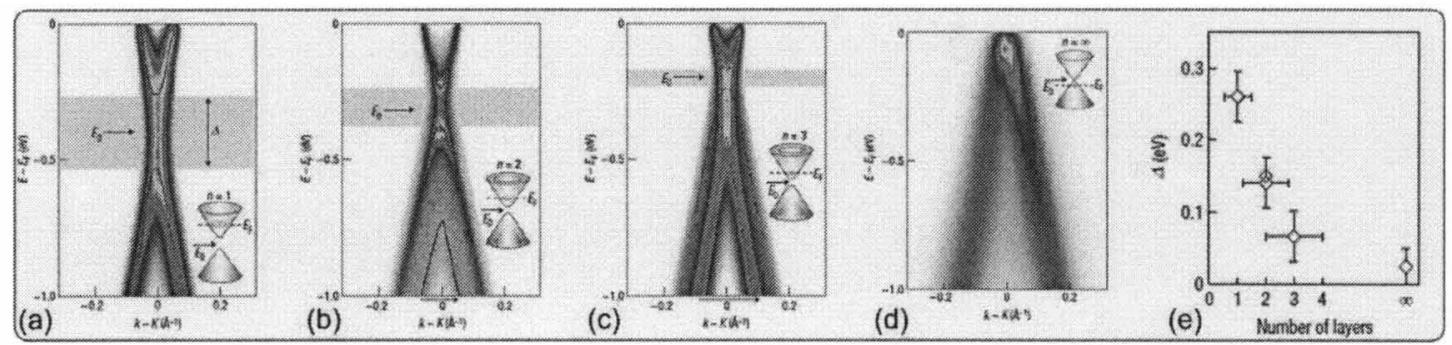

Figure 3.24 (a)-(d) ARPES measurements presenting the decrease in band gap with increase in number of layer grown. (e) Plot showing the energy band gap value versus number of layers. (adapted from [12]).

Again, the band gap due to substrate induced effects is too small to be of any practical use at room temperature.

The zero band gap in graphene is a direct consequence of the identical potential of the two carbon atoms in the graphene unit cell. It is predicted that just by creating a potential mismatch on the two atoms a gap can be opened. A chemical reaction that forms a superstructure by altering the bonding pattern introduces a potential mismatch on the two carbon atoms. Because of this motivation, graphene functionalization with oxygen has generated a lot of attention. Graphene oxide synthesis and its electrical and optical characterization have been carried out extensively since the introduction of monolayer 
graphene in 2004. Attempts to oxidize graphene following methods used for graphite oxidation (e.g. Hummer's method), lead to heavy oxidation of graphene, which in turn converted it into wide gap semiconductor (insulator).

Experimental evidence of controlling the conductivity of $\mathrm{GO}$ by reduction processes lead to extensive theoretical study of analyzing the effect of oxygen content on the conductance. Figure 3.25 summarizes some of the theoretical results, suggesting that the GO electronic density of states (DOS) varies non-monotonically with energy, and contains numerous gaps and peaks (gaps are present even in the valence band). The exact form of the DOS is predicted to depend on the oxygen coverage on the graphene surface. Boukhvalov et al. has predicted that only some configuration of oxidized graphene is stable and the DOS of these states will be dependent on the oxygen coverage percentage. Lahaye et al. predicts a separated DOS for majority and minority spins for partially oxidized graphene. The separation in spin DOS may be exploited in magnetic devices. Theory predicts the possibility of partial oxidation and the dependence of band gap opening on the oxygen coverage. For certain oxygen concentrations, semiconducting behavior is expected. This implies the possibility of tuning the GO conductivity by varying the oxygen concentration.

During the course of this dissertation study, hydrogenation and fluorination of graphene have emerged as new ways of functionalization to open a band gap. However, fluorographene behaves as a high quality insulator (resisitivity $>10^{12} \Omega$ ) with an optical gap of $3 \mathrm{eV}$. On the other hand, hydrogenated graphene (or graphane) has shown promise as an alternate way to open band gap. Initial measurements and calculations predict a small gap of $0.5 \mathrm{eV}$ for partially hydrogenated samples. 


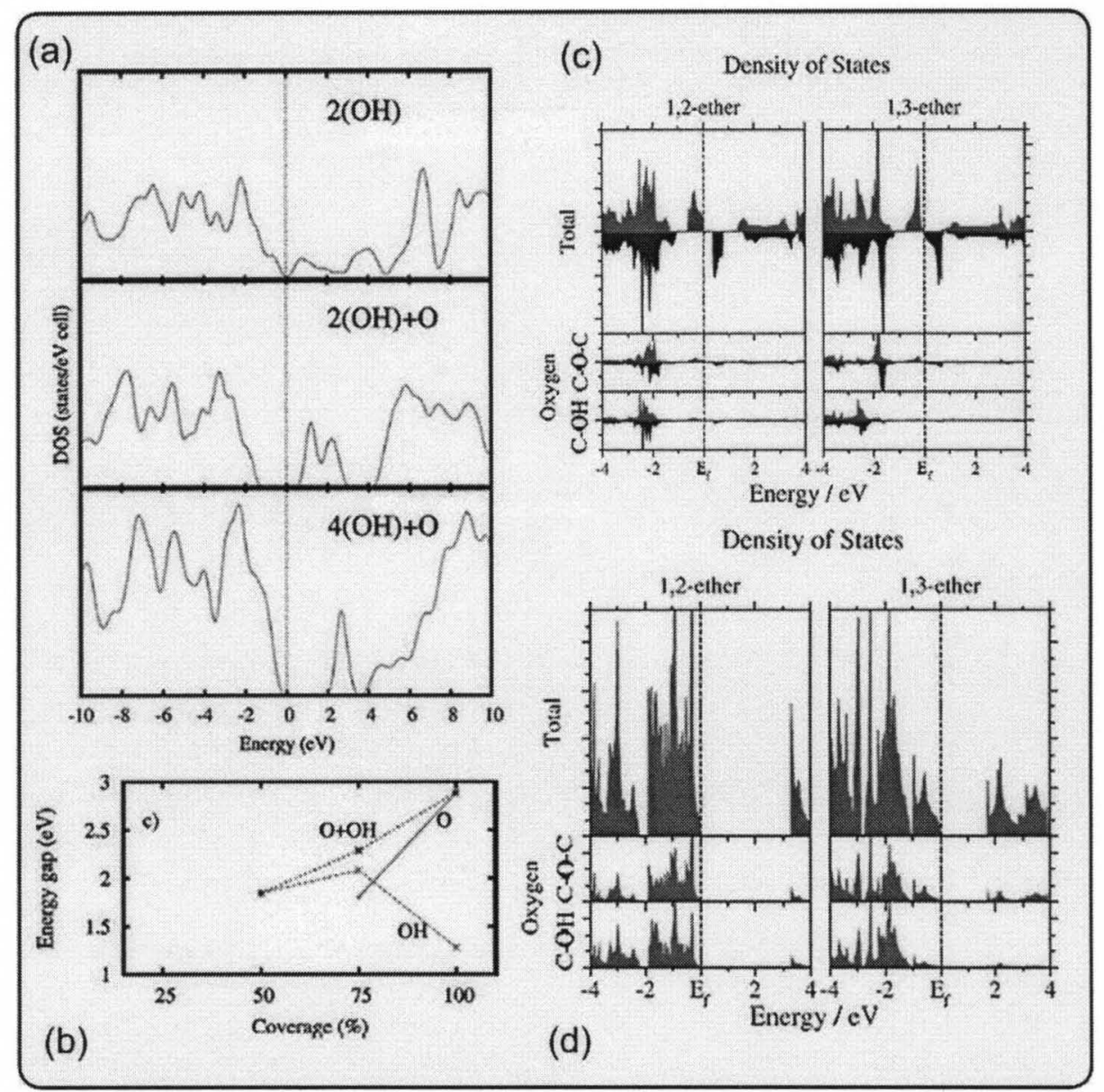

Figure 3.25 (a)-(b) Adapted from Boukhlov et al., [19] (a) three stable configuration of oxidized graphene with respective DOS. (b) Change in energy gap with change in oxygen coverage percentage. (c)-(d) Adapted from Lahaye et al., [20] (c) DOS of partially oxidized graphene, with the majority (red) and minority (blue) spins separated. (d) DOS of fully oxidized graphene.

Conductance measurements on their own do not provide any strong evidence for an optically accessible band gap, because scattering from physisorbed and chemisorbed oxygen atoms, or oxygen doping (particularly in the presence of a substrate induced gap) can also influence the graphene conductance. Without a direct measure of the optical band-gap, it is difficult to distinguish between these different scenarios. On the other 
hand, optical measurements (absorption and PL) have provided only limited information regarding the energy states in GO samples.

As discussed, after considering various approaches followed so far for opening a band gap in graphene, controlled oxidation of graphene looks most promising. The previous discussion also demonstrates the need for an experimental method of observing the density of states of the resultant functionalized product.

In the next chapter, a pristine way of oxidation and a novel approach (capacitive photocurrent spectroscopy) towards the measurement of density of states (DOS) of graphene oxide is presented. 


\section{CHAPTER IV}

\section{PRELIMINARY MEASUREMENTS AND CAPACITIVE PHOTOCURRENT SPECTROSCOPY}

\subsection{Initial Measurements}

As discussed in the last chapter, methods used to create GO were derived from graphite oxidation techniques, which produce strong oxidation of graphene pieces. Reduction of this GO provides one way to vary the oxygen content of the sample. Control on the oxygen coverage to increase the conductivity is achieved by controlling the reducing parameters (such as time of treatment, temperature). Since, theory calculations suggest that progressive oxidation is possible; this dissertation presents a different approach of controlling carbon/oxygen ratio by slowly increasing the oxidation content. This method involves treatment of graphene with Brønsted acid $\left(\mathrm{H}_{2} \mathrm{SO}_{4}+\mathrm{HNO}_{3}\right)$ at room temperature and the treatment time is used to provide control on the oxidation coverage. This allows a more gradual oxidation of the graphene than is possible using the more standard Hummers method.

To confirm that the treatment of graphene with Brønsted acid will result in oxidation, a small piece of natural occurring KISH graphite is treated with a mixture of 
$5 \mathrm{ml}$ sulfuric acid $\left(\mathrm{H}_{2} \mathrm{SO}_{4}, 99 \%\right.$ concentrated $)$ and $5 \mathrm{ml}$ nitric acid $\left(\mathrm{HNO}_{3}, 70 \%\right.$ concentrated). An increase in volume of the KISH graphite piece was observed upon treatment. XPS measurements were performed to check the presence of oxidized functionalized group attached to the carbon atoms in the graphite piece. X-ray photoelectron spectroscopy (XPS) data for the KISH and the oxidized KISH samples are presented in Figure 4.1.

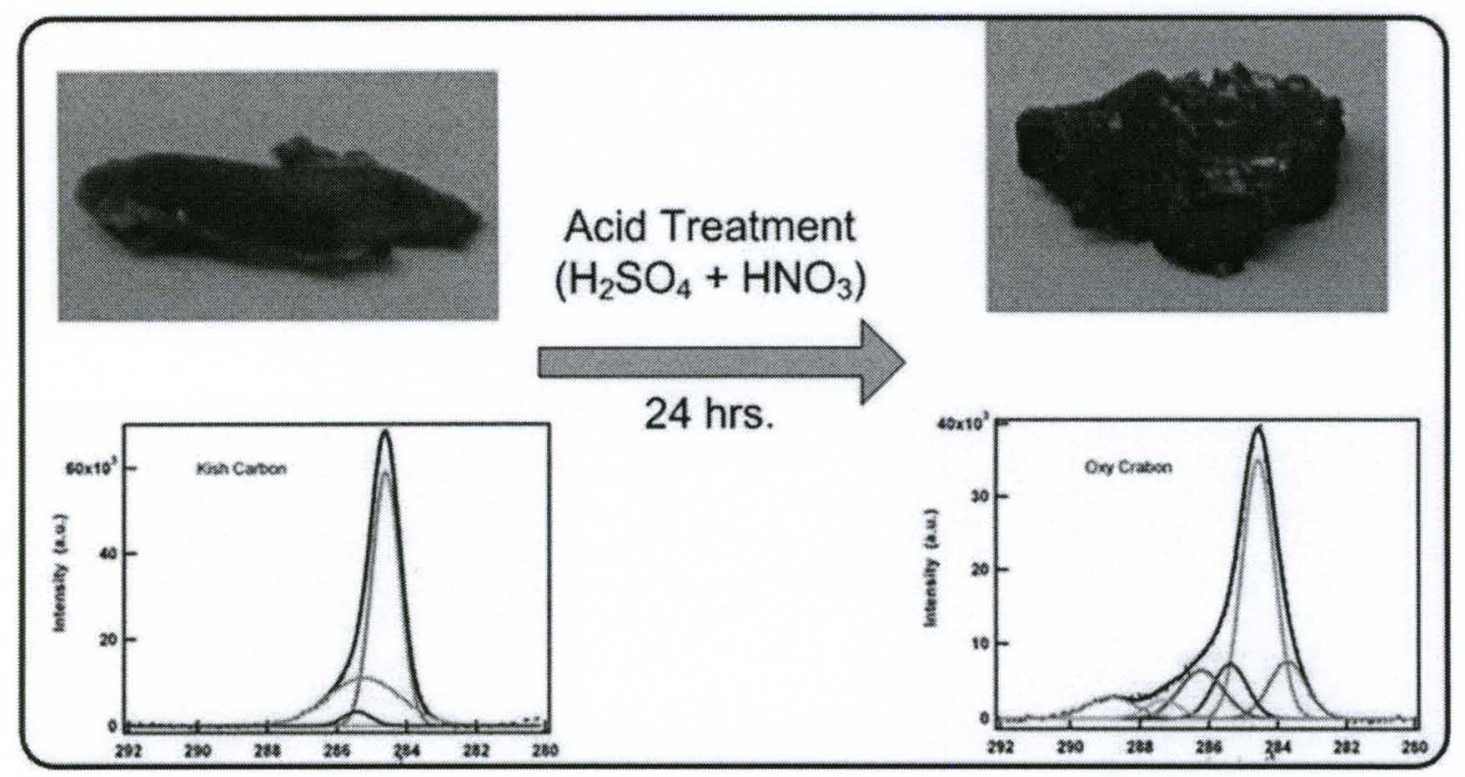

Figure 4.1 Optical image and XPS C1s curves for KISH graphite before and after 24 hours of acid treatment.

After subtracting a constant background, X-ray photoelectron spectra of the carbon 1s level are de-convoluted into various components using Gaussian peak line shapes. As reported in the literature, the resulting de-convoluted peaks can be attributed to the different functional groups of the carbon atom. Both the natural KISH graphite sample and the oxidized KISH graphite sample show evidence for the graphitic non-oxygenated $\mathrm{C}$ ring $(\mathrm{C}-\mathrm{C}, \sim 284.6 \mathrm{eV})$ and the $\mathrm{C}-\mathrm{O}$ single bond $(\sim 286.2 \mathrm{eV})$. In the oxidized sample 
two extra features for the $\mathrm{C}=\mathrm{O}$ double bond $(\sim 287.8 \mathrm{eV})$ and the $\mathrm{O}-\mathrm{C}=\mathrm{O}$ groups $(\sim 289.1$ eV) are also observed. The peak intensity varies considerably between the two samples, with the peaks associated with the oxygen groups being stronger in the oxidized KISH sample than in the natural KISH sample.

To measure the electrical properties of the oxidized graphene, mechanical exfoliation approach to obtain mono-layer graphene was employed. Figure 4.2 provides the flow chart of the fabrication procedure. Optical images of the graphene sample at various fabrication steps is shown in Figure 4.3.

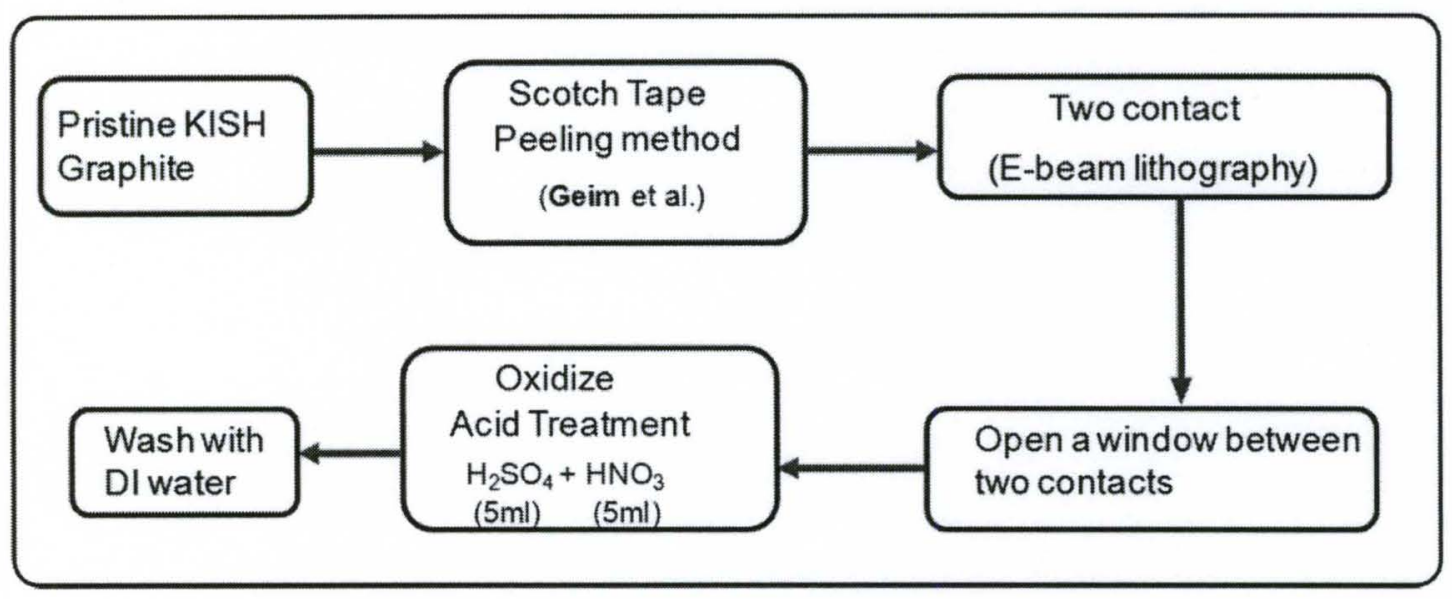

Figure 4.2 Flow chart of the device fabrication procedure for electrical measurements.

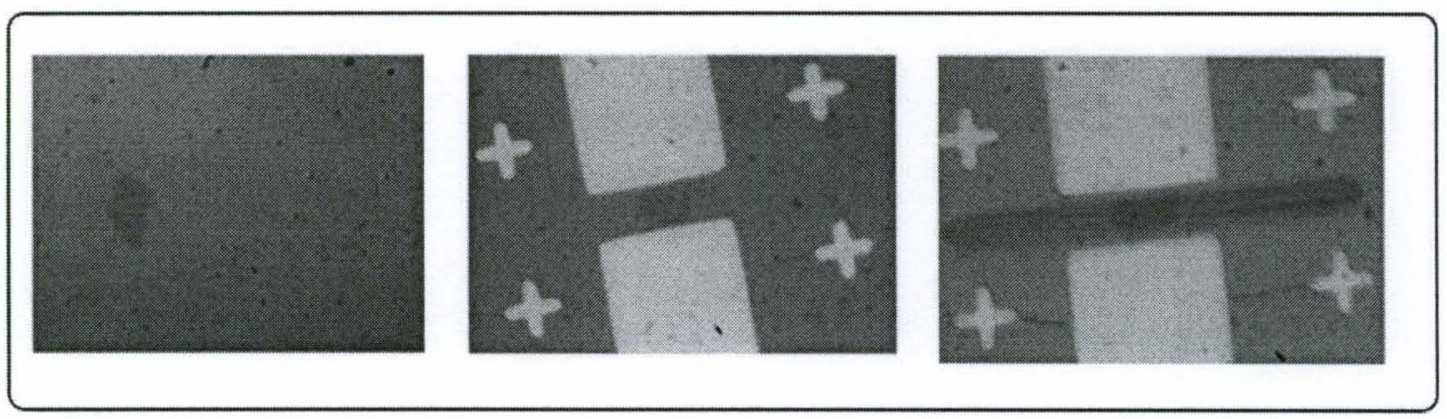

Figure 4.3 Optical image of graphene piece lying on the heavily doped $\mathrm{Si} / \mathrm{SiO}_{2}$ substrate. Patterned etch marks near the graphene piece shows a high resolution grid pattern used for locating the piece on the substrate for e-beam lithography. 
Figure 3.4 (a) shows a freshly peeled graphene piece lying on the high resolution grid area on a $\mathrm{SiO}_{2} / \mathrm{Si}$ substrate. Heavily doped (p-type) $\mathrm{Si}$ together with $300 \mathrm{~nm} \mathrm{SiO}_{2}$ will act as the metal oxide back gate for the graphene device. Figure 3.4 (b) shows the e-beam lithography patterned Ti/Au electrodes and for electrical measurements. Figure 3.4 (c) shows a window opening in the e-beam resist covering the metal electrodes which allow oxidation of only the graphene piece without affecting the properties of contact electrodes due to presence of acids. Figure 4.4 presents the gate dependence measurements of the graphene device before and after the oxidation step.

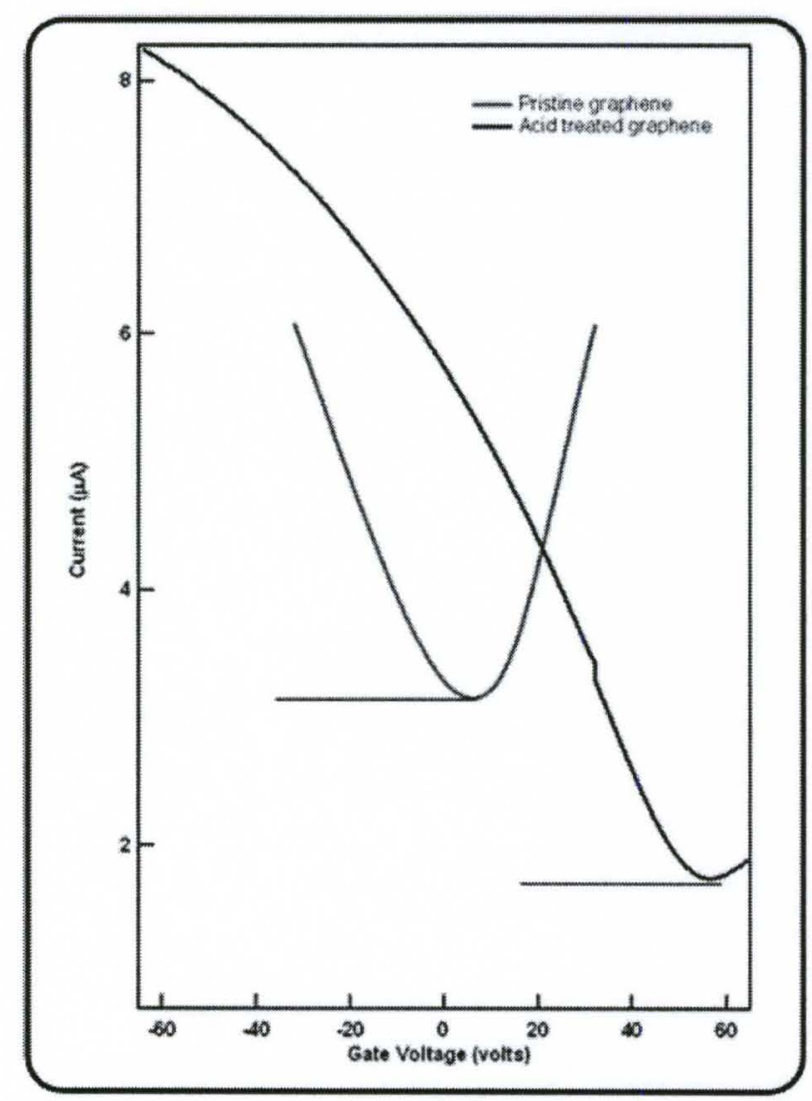

Figure 4.4 Gate dependence measurements on graphene piece before and after acid treatment (oxidation) step. 
After the oxidation step the Dirac point shifts to higher gate voltage and the measured current value decreases at the Dirac point. The shift suggests an increase in p-type behavior due to oxidation or charge trapping in the $\mathrm{SiO}_{2}$ layer during the acid treatment. The reduction in the current value at the Dirac point can be interpreted as a decrease in the DOS near the Fermi energy. Another, possible reason for the reduction in current is an increase in scattering due to defect formation during oxidation. The resistance of the graphene device increased from $\sim 7 \mathrm{~K} \Omega$ to $15 \mathrm{~K} \Omega$ after oxidation. Since a finite amount of current is flowing for all the gate voltages, no evidence of band gap opening is observed.

Initial XPS measurements proved the presence of oxygen functionalized group attached to the carbon atoms after the acid treatment and the electrical measurements presented the influence of these groups on the device characteristic measurements.

\subsection{Capacitive Photocurrent Spectroscopy}

This section introduces the capacitive photocurrent technique and its detailed experimental setup.

\subsubsection{Experimental Setup}

The basic measurement setup is as shown in Figure 4.5. A parallel plate capacitor configuration is achieved where the nano-material (graphene, GO or rGO) under investigation acts as one of the parallel plate and the copper block anchored to the measurement stick within an optical access flow cryostat acts as the other conducting plate. The nano-material is deposited on a quartz slide which is attached to the copper 
block in a way that will eliminate any direct electrical connection between the nanomaterial and the copper block (fulfilling the basic requirements of a capacitor design). For connecting these capacitor plates to the measurement circuitry, two electrodes were created. A transparent metal contact is deposited on the nonmaterial covering the entire substrate surface which act as the first electrode while the copper block is in direct electrical contact with the measuring stick making it the second electrode. Using Indium tin oxide (ITO) as a transparent metal contact to the nano-material eliminates the need for locating the nano-material on the quartz slide. For the case of nano-material films a direct electrical contact can be made by attaching an electrical wire at the corner of the film surface using colloidal silver paint. The configuration with ITO as the contact metal is preferred, even for the case of nano-material films, because of its increased sensitivity and larger signal amplitude.

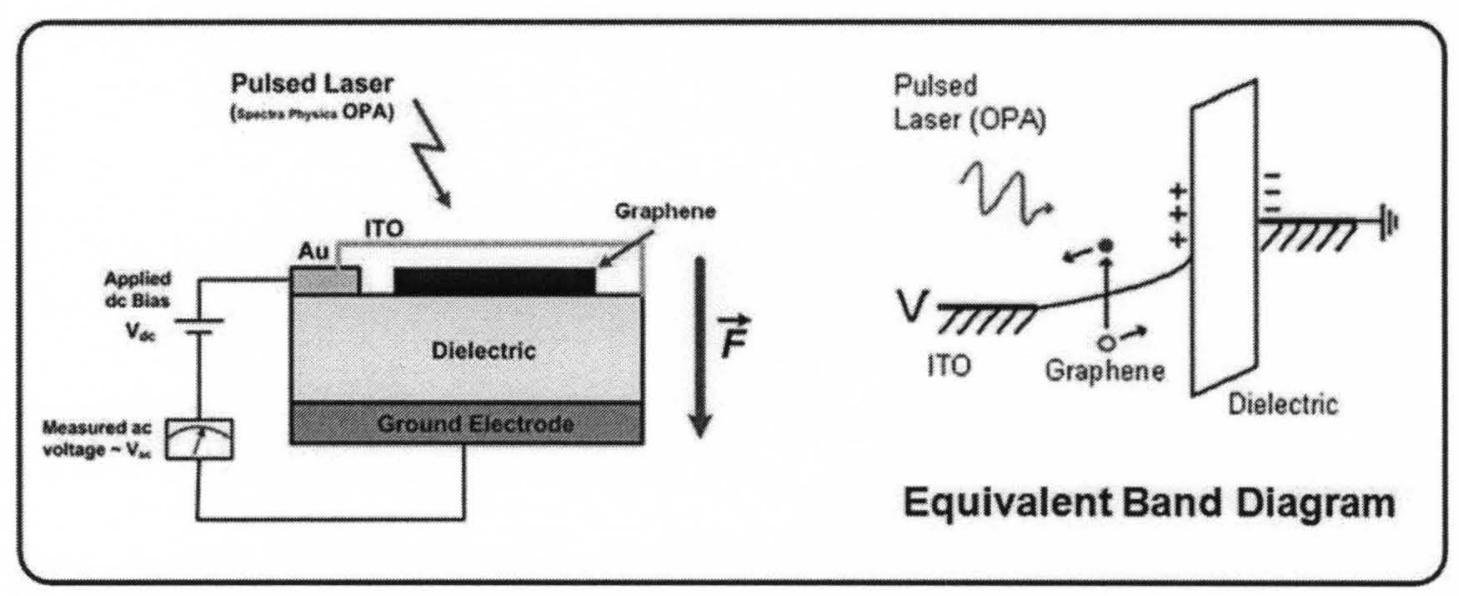

Figure 4.5 Figure shows the basic setup for the capacitive photocurrent spectroscopy technique. The photocurrent is determined by measuring the output voltage using a lockin amplifier set $90^{\circ}$ out of phase from the pulsed laser repetition frequency. The dc bias produces an electric field across which help in enhancing the signal. 
Also, the signal using ITO electrode is very stable, as compared to direct contact by silver paste, and can be amplified further by applying an external dc voltage.

The operational principle of CPS can be understood with the illustration of its detection method as shown in Figure 4.6. The electrode potential Vp is measured relative to the graphene, which is held at ground. With the absence of light, $\mathrm{Vp}$ is given by the work function difference between the graphene and the electrode, and any additional built-in potential which may be present due to the trapped charge within the quartz or graphene. The band diagram is similar to a MOS capacitor, with the semiconductor replaced by the graphene.

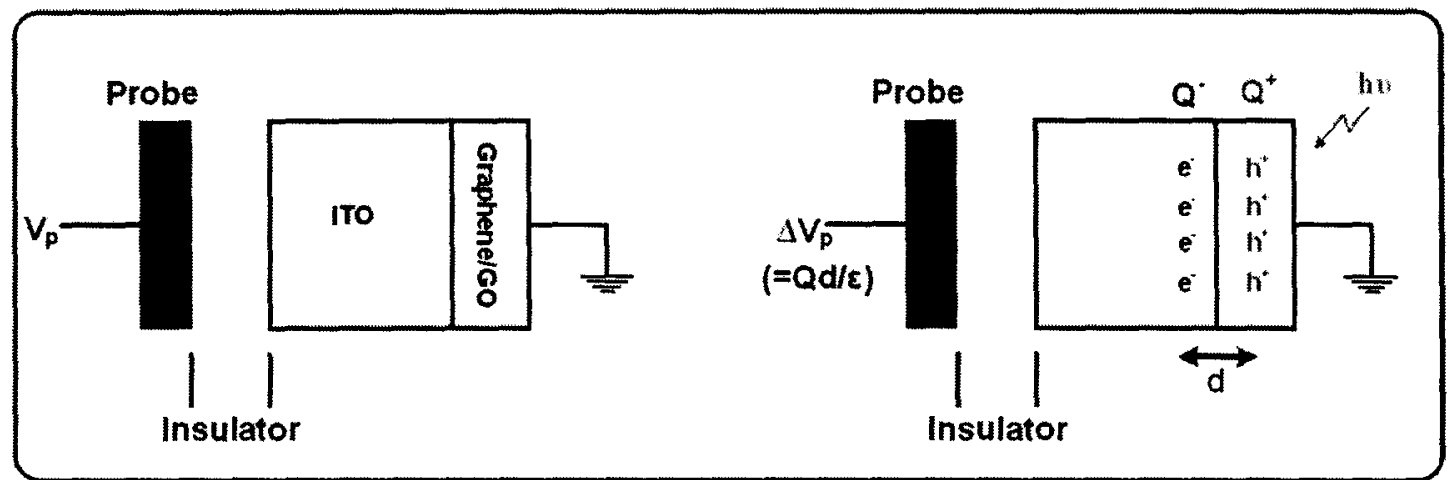

Figure 4.6 Cross-section of ITO/Graphene heterojunction monitored by a capacitively coupled probe electrode with (a) no light and (b) light on the sample.

Light incident on the sample excites electron hole pairs. If the excited electron/hole pair (ehps) separate, due to the internal and/or external electric field, an additional potential is created, which to first approximation is given by:

$$
\Delta V_{p}=\frac{Q d}{\varepsilon}
$$


where $Q$ is the total charge per area generated, and $d$ is the charge separation distance. Since the probe electrode is floating, its potential increases by an amount $\Delta \mathrm{Vp}$ to match the potential change. This measured voltage reflects the absorbance in the graphene.

Since ITO is used as transparent metal, its transparency characteristics need to be known. Figure 4.7 [70] shows the transparency for $30 \mathrm{~nm}$ ITO on quartz substrate to be more than $90 \%$ for the energy range scanned $(0.5 \mathrm{eV}$ to $3 \mathrm{eV})$.

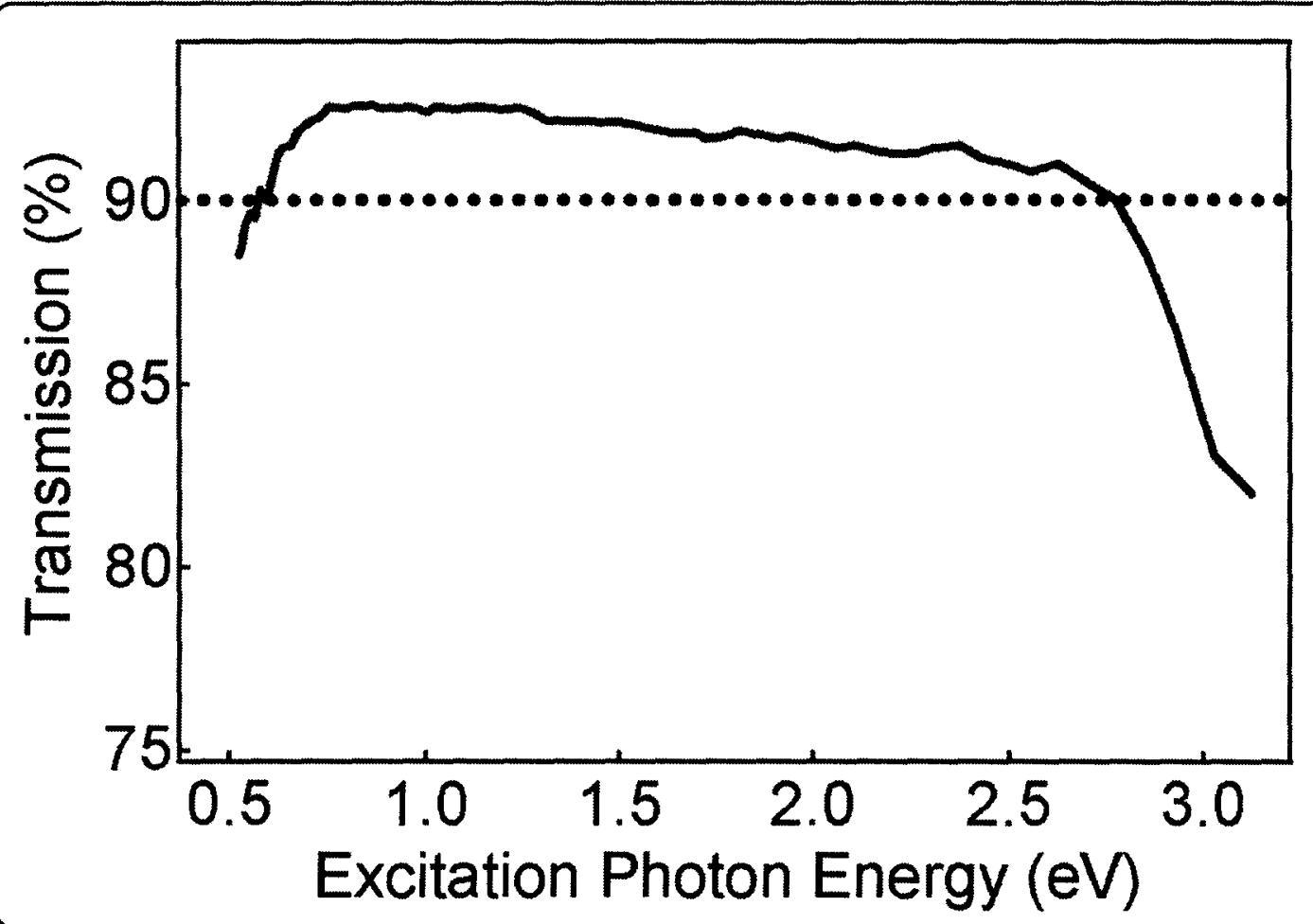

Figure 4.7 Optical transmission through a $30 \mathrm{~nm}$ film of ITO on quartz shows $>90 \%$ transmission between $0.5 \mathrm{eV}-3 \mathrm{eV}$. (adapted from [70]).

Preliminary measurements were performed on a p-type silicon sample to compare the CPS results with standard two terminal DC photocurrent measurements. Figure 4.8 plots the capacitive photocurrent as a function of excitation photon energy. The photocurrent is relatively low until the excitation energy is in the vicinity of the band gap of silicon (1.1 
$\mathrm{eV})$. When the excitation energy equals the band gap energy, the photocurrent increases and remains relatively constant. The turn on point of the photocurrent can be quite accurately matched with the absorption spectrum for p-type silicon which confirms the availability of CPS as a standard spectroscopy technique.

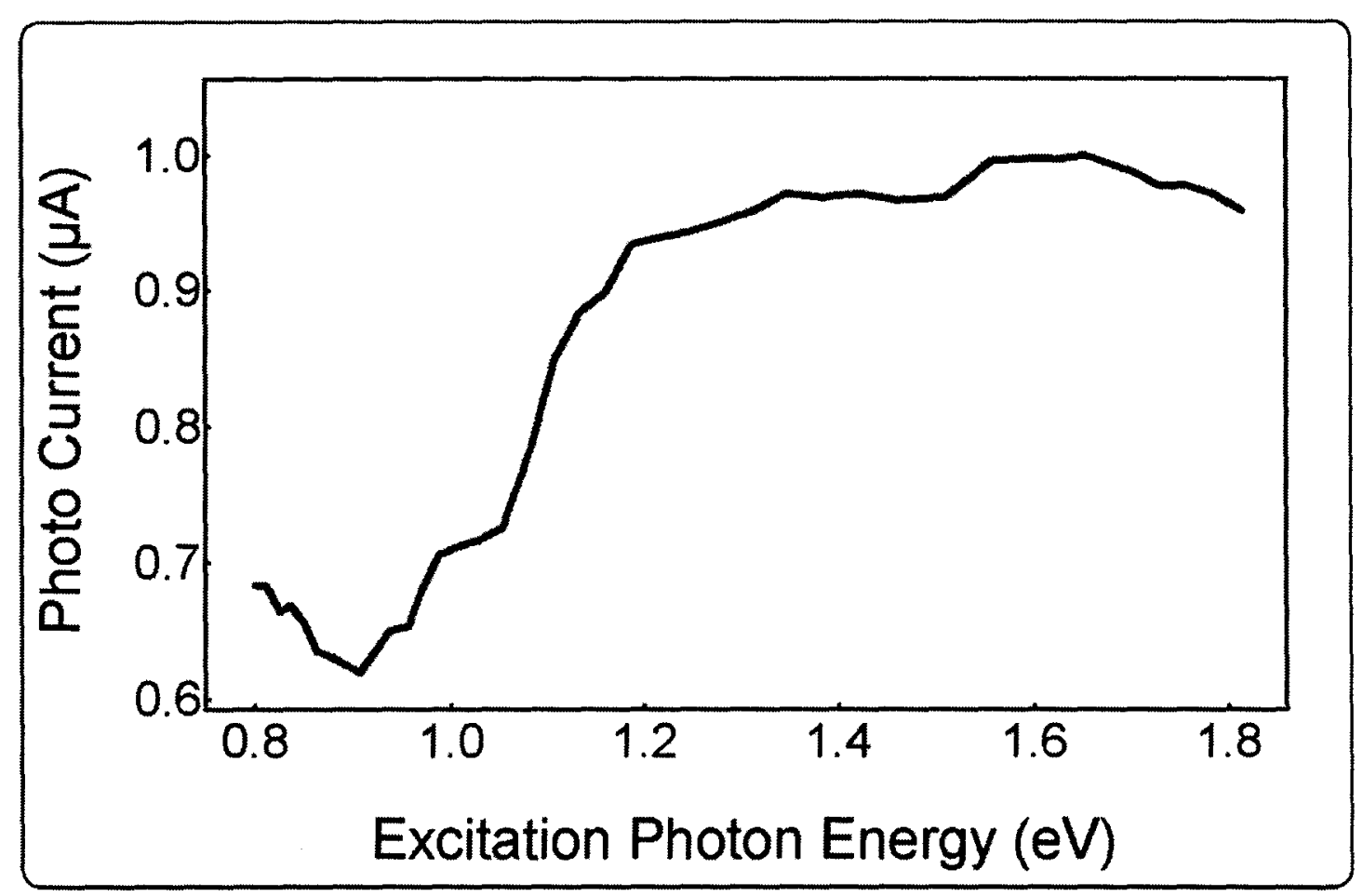

Figure 4.8 Capacitive photocurrent response of a p-Si plotted as a function of excitation photon energy shows the typical band edge of silicon at $\sim 1.1 \mathrm{eV}$. (adapted from [70]).

\subsection{Optical Source}

The optical source used in the experiments was a Spectra Physics femto second pulsed laser system coupled with an optical parametric amplifier (OPA) to tune its wavelength over a very broad range $(300-2400 \mathrm{~nm})$. The femto second pulse train system consist of an oscillator, a stretcher, an amplifier and a compressor combined as a 
system package called "Hurricane" as shown in Figure 4.9, while the OPA feeds on the output of the Hurricane to provide the wavelength tuning capabilities.

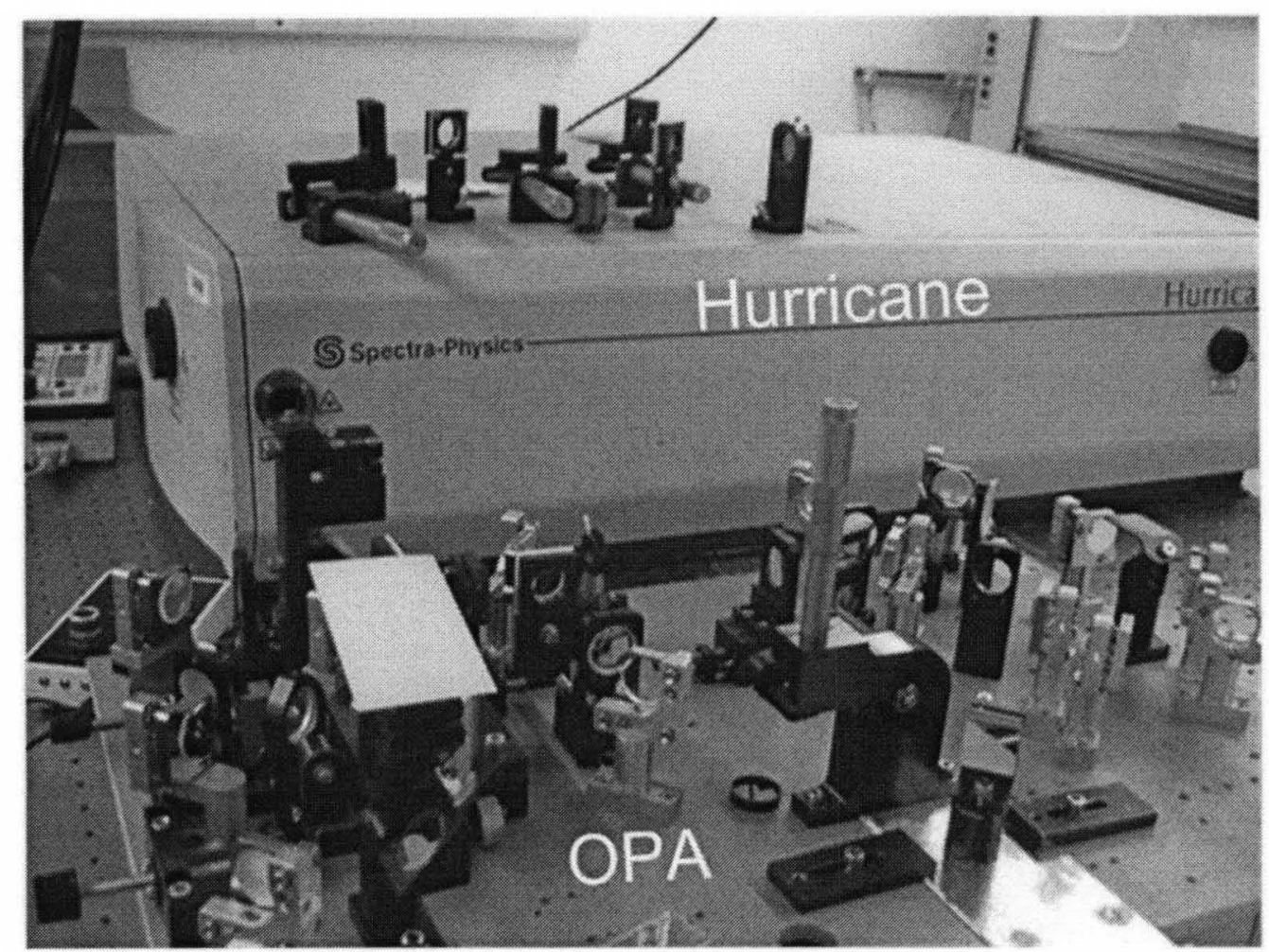

Figure 4.9 A digital camera image of the OPA aligned next to a Hurricane which pumps the OPA.

In general, generation of a typical high power femto second chain includes an oscillator that produces $100 \mathrm{fs}$ pulses at a repetition rate of typically $100 \mathrm{MHz}$. After passing through the stretcher the pulse duration increases up to $400 \mathrm{ps}$ and can be amplified. The gain medium is a Ti:sapphire (titanium-doped sapphire) crystal pumped by the second harmonic of a Q-switched Nd:YAG running at $1 \mathrm{KHz}$. Once trapped in the cavity the pulse is amplified at each pass in the crystal with a typical gain per pass of 2 . After 15-20 round trips the pulse saturates the gain and reaches its maximum energy. It is extracted from the amplifier and sent into multi pass power amplifier and finally into the 
compressor. There it recovers its initial duration and pulses as short as $30 \mathrm{fs}$ with energies in the joule range can be obtained [71].

The Spectra Physics Hurricane system used in this dissertation consists of four subsystems: seed laser Mai Tai, pump laser Evolution, stretcher/compressor and regenerative amplifier as shown in Figure 4.10 [72].

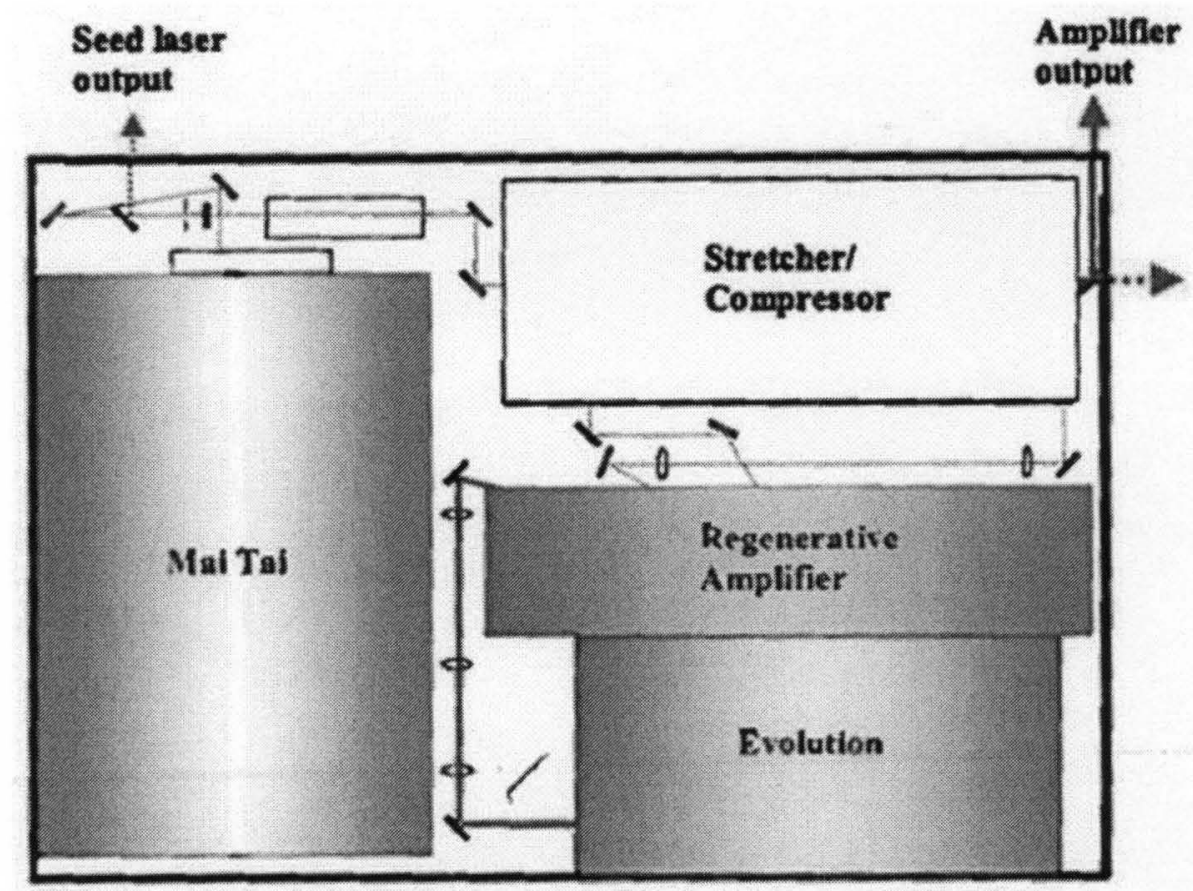

Figure 4.10 The four subsystems of Hurricane: Mai Tai, Evolution, stretcher/compressor and regenerative amplifier [72].

Mai Tai, in itself, is comprised of two chambers, A continuous wave (CW) pump chamber and a pulsed output chamber containing two lasers, a CW diode-pumped laser and a mode-locked Ti:sapphire pulsed laser respectively. The CW diode pump laser is an all solid-state, high power $(5 \mathrm{~W})$, visible $\mathrm{CW}(532 \mathrm{~nm})$ single phase laser. The output of the laser diode $(825 \mathrm{~nm})$ pumps the $\mathrm{Nd}^{3+}$ ions doped in a yttrium vanadate crystalline 
matrix (Nd: YVO4) which start lasing at $1064 \mathrm{~nm}$ due to the population inversion in the crystal matrix. The resulting $1064 \mathrm{~nm}$ output is converted to visible $(532 \mathrm{~nm})$ through a frequency doubling or second harmonic generation using a $90^{\circ}$, noncritically phase matched, temperature-tuned lithium triborate (LBO) nonlinear crystal as doubling medium. The pulsed chamber uses this $\mathrm{CW}$ laser $(532 \mathrm{~nm})$ output as the pump beam for the Ti:sapphire amplifier fitted with an acousto-optic modulator for mode locking purposes. In a mode locked laser the longitudinal modes are locked in phase such that they constructively interfere to create a single circulating pulse. An output pulse is generated every $12.5 \mathrm{~ns}$ which corresponds to the arrival of the intra-cavity pulse at the partially reflective output coupler. Collectively, Mai Tai delivers a low power $(\sim 0.75 \mathrm{~W}$ to $.90 \mathrm{~W})$ femto-second $(\sim 120 \mathrm{fs})$ mode locked continuously tunable pulsed output over a range of near infrared wavelengths from $750 \mathrm{~nm}$ to $850 \mathrm{~nm}$ at a repetition rate of $80 \mathrm{MHz}$ $[73]$.

The rest of the three subsystems namely stretcher/compressor, evolution and regenerative amplifier collectively constitute the chirped pulse amplification system which increases the power of the pulsed laser beam keeping the width of the pulse and mode-locking intact. Using a single grating stretcher, a low energy, very short duration pulse is stretched to as much as 10,000 times. This stretched pulse is then amplified using a Ti:Sapphire regenerative amplifier which can increase the pulse energy as much as $10^{6}$ times. A single grating compressor recompresses the pulse back to its original duration.

The OPA consists of a single optical head that feeds on the output of the Hurricane amplifier (Figure 4.9). The infrared wavelength extension is achieved in a two-step process with white light generation and traveling-length optical parametric amplification. 
The output wavelength is tuned by changing the angle of the $\beta$-Barium Borate (BBO) crystal. Wavelength tuning in the visible and near IR regions is achieved using the integrated harmonic generation (HGI and HGII).

Although the OPA was used to perform all the measurements described in this dissertation, the capacitive photocurrent technique also works with a $\mathrm{CW}$ (continuous wave) laser in combination with a chopper and has also shown promise with white light system being the optical source. 


\section{CHAPTER V}

\section{MEASUREMENT OF DENSITY OF STATES OF GRAPHENE OXIDE AND REDUCED GRAPHENE OXIDE}

This dissertation provides the measure of the density of states (DOS) for CVD grown graphene, GO, and rGO using a new measurement technique called capacitive photocurrent spectroscopy, or CPS. While similar to absorption spectroscopy, CPS is unique in that it specifically measures absorption due to the formation of charge carriers, while being uninfluenced by absorption due to vibrational states of the lattice, or nonmobile impurity states $[73,74]$. Due to this, the capacitive photocurrent spectrum reveals previous unobserved details of the GO DOS. The capacitive photocurrent spectrum of graphene increases monotonically with increasing energy (as expected from the semimetallic graphene DOS) together with three reproducible peaks. For GO, the monotonic background disappears, and the three peaks become the dominant features in the spectrum. Two of these peaks match well with published PL data, as transitions between the GO states and the $\pi / \pi^{*}$ graphene levels match the peak luminescence energy of GO. Reduction of the GO results in the re-emergence of the background density of states due to the graphene, along with additional states due to defects, The three peaks present due to the GO also remain, indicating the continued presence of oxidized regions. 


\subsection{MEASUREMENTS AND RESULTS}

X-ray photoelectron spectroscopy (XPS) data for the CVD grown graphene, graphene oxide (GO) and reduced graphene oxide (rGO) samples are presented in Figure 5.1. After subtracting a constant background, X-ray photoelectron spectra of the carbon 1s level are de-convoluted into various components using Gaussian peak line shapes. As reported in the literature, the resulting de-convoluted peaks can be attributed to the different functional groups of the carbon atom $[54,75]$. The GO, $\mathrm{GGO}$, and graphene sample all show evidence for the graphitic non-oxygenated $\mathrm{C}$ ring $(\mathrm{C}-\mathrm{C}, \sim 284.6 \mathrm{eV})$, the $\mathrm{C}-\mathrm{O}$ single bond $(\sim 286.2 \mathrm{eV})$, the $\mathrm{C}=\mathrm{O}$ double bond $(\sim 287.8 \mathrm{eV})$ and the $\mathrm{O}-\mathrm{C}=\mathrm{O}$ groups $(\sim 289.1$ $\mathrm{eV}$. The peak intensity varies considerably between the different samples, with the peaks associated with the oxygen groups being stronger in the GO sample than in the CVD grown graphene or the rGO. In addition, the rGO sample has one extra component at $285.9 \mathrm{eV}$, indicating the presence of a carbon-nitrogen group. The nitrogen is presumably introduced during the reduction process. 


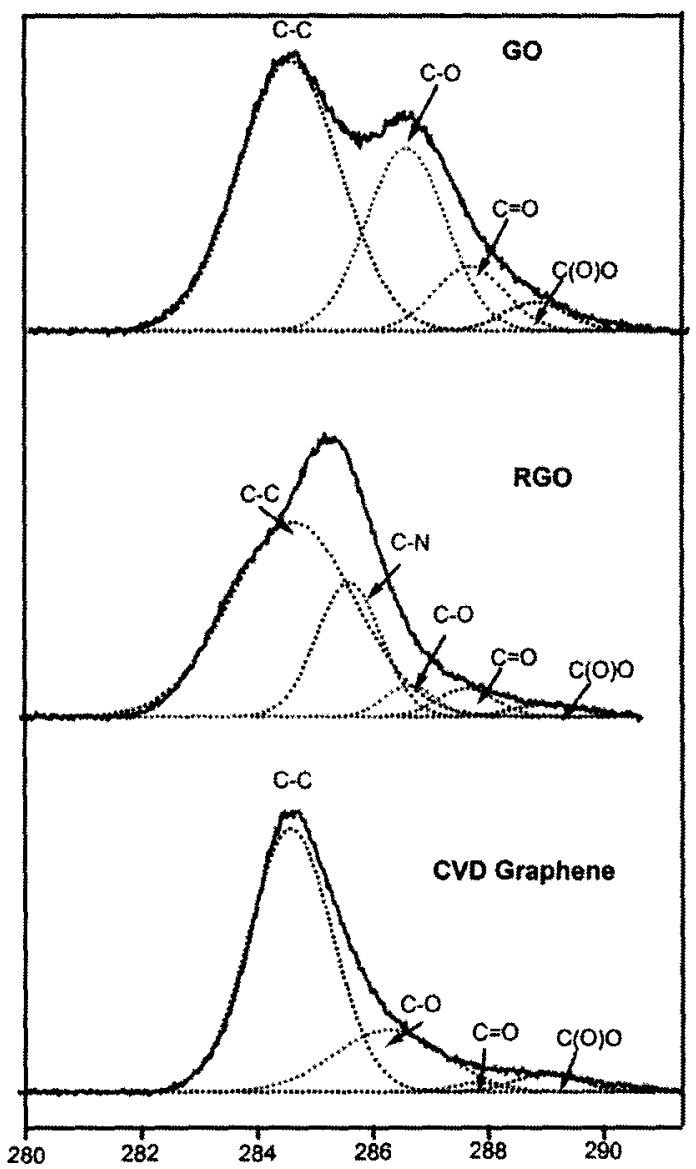

Figure 5.1. X-ray photoelectron spectra (red curves) of GO, rGO and CVD grown graphene samples with de-convoluted components (light colored curves).

Capacitive photocurrent measurements were then performed using the set-up shown in Figure 5.2. The sample lies on top of an ITO coated quartz slide, which is anchored to a copper plate within an evacuated optical cryostat. Electrical contact is made between the copper plate and a gold pad deposited near the edge of the ITO layer. The sample is illuminated with light from an optical parametric amplifier (OPA) which emits a series of $120 \mathrm{fs}$ light pulses at a $1 \mathrm{kHz}$ repetition rate. The output power is kept constant at $5 \mathrm{~mW}$, and the photon energy is tuned between 0.5 and $4.0 \mathrm{eV}$. The ITO and quartz are both transparent to light within this energy range. If light absorbed by the graphene produces 
electron-hole pairs, these separate across the graphene / ITO interface under the influence of an applied dc potential, $\mathrm{V}_{\mathrm{dc}}$. This produces an ac voltage, $\Delta \mathrm{V}$, whose magnitude is proportional to the amount of excited charge, multiplied by the charge separation distance, $\mathrm{d}$, and whose frequency is equal to the laser repetition rate. The voltage is converted to a current through a current amplifier, and detected with a lock-in amplifier synched to the laser repetition rate.
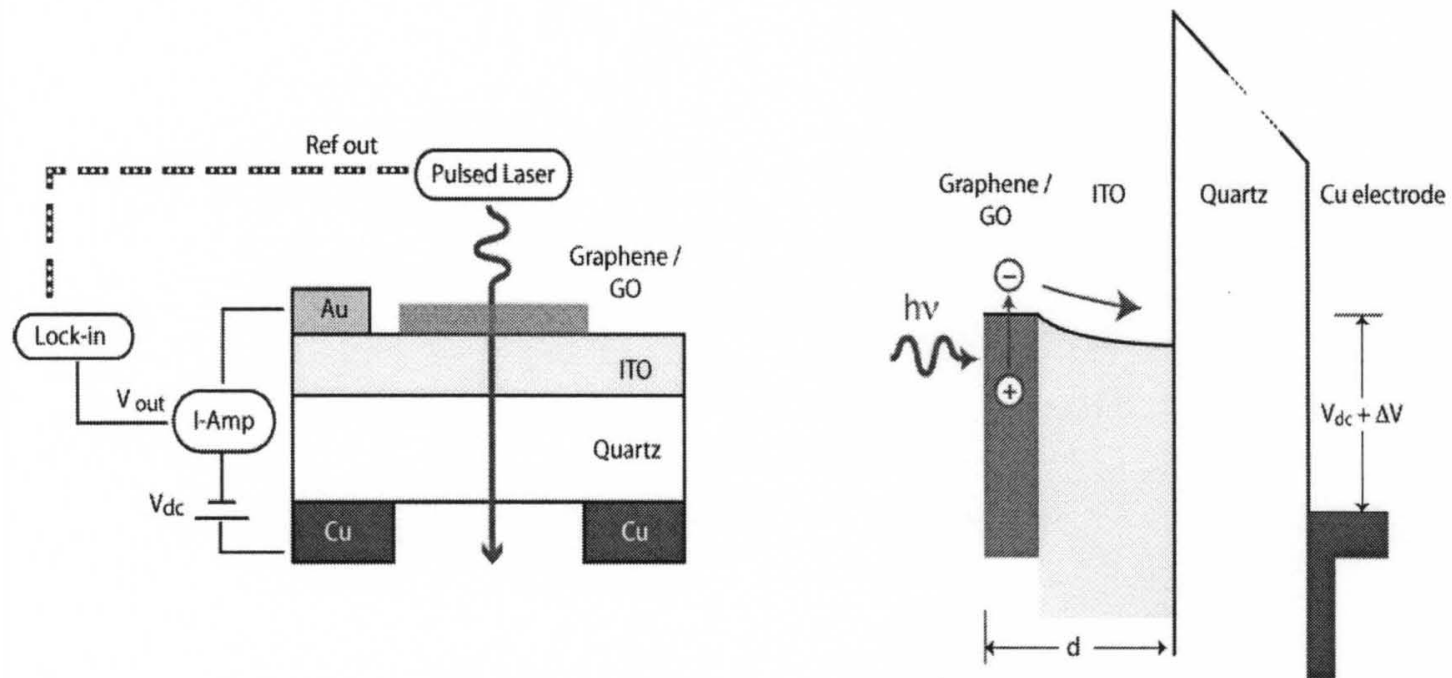

Figure 5.2. Schematic of the experimental setup (left) and the equivalent band diagram (right) for the CPS measurement.

Figure $5.3(a-d)$ shows the capacitive photocurrent as a function of excitation energy for four different individual graphene sheets. The raw photocurrent data is normalized in units of electrons/photon (or quantum yield) by dividing the measured photocurrent (in electrons/s) by the number of photons/s (determined by dividing the laser power by the excitation energy). Graphene sheets in (a) and (b) were lifted off using $10 \%$ aqueous solution of $\mathrm{HNO}_{3}$ while those in (c) and (d) were lifted off using ferric chloride solution to avoid oxidation. In each measurement, there is a monotonic increase in the 
photocurrent as a function of excitation energy, with fluctuations superimposed on the background signal. Peaks near $0.7 \mathrm{eV}, 1.6 \mathrm{eV}$ and $3.2 \mathrm{eV}$ (marked with $\boldsymbol{\Delta}$, $\bullet$, and $\mathbf{m}$, respectively) each reproduce between at least two samples, showing that they are not noise, and occur independently of the lift-off procedure. To test the influence of the ITO contact, two of the samples ((a) and (c)) were measured using the standard measurement configuration shown in Figure 5.3 (a), while the other two (b) and (d) were measured with the graphene lying on quartz, with a remote silver paste contact placed on a corner of the graphene sheet (this reduces the magnitude of the signal somewhat). The peak at $0.7 \mathrm{eV}$ (marked with $\boldsymbol{\Delta}$ ) occurs only in the samples on ITO, suggesting that it might be enhanced by the graphene / ITO interface.
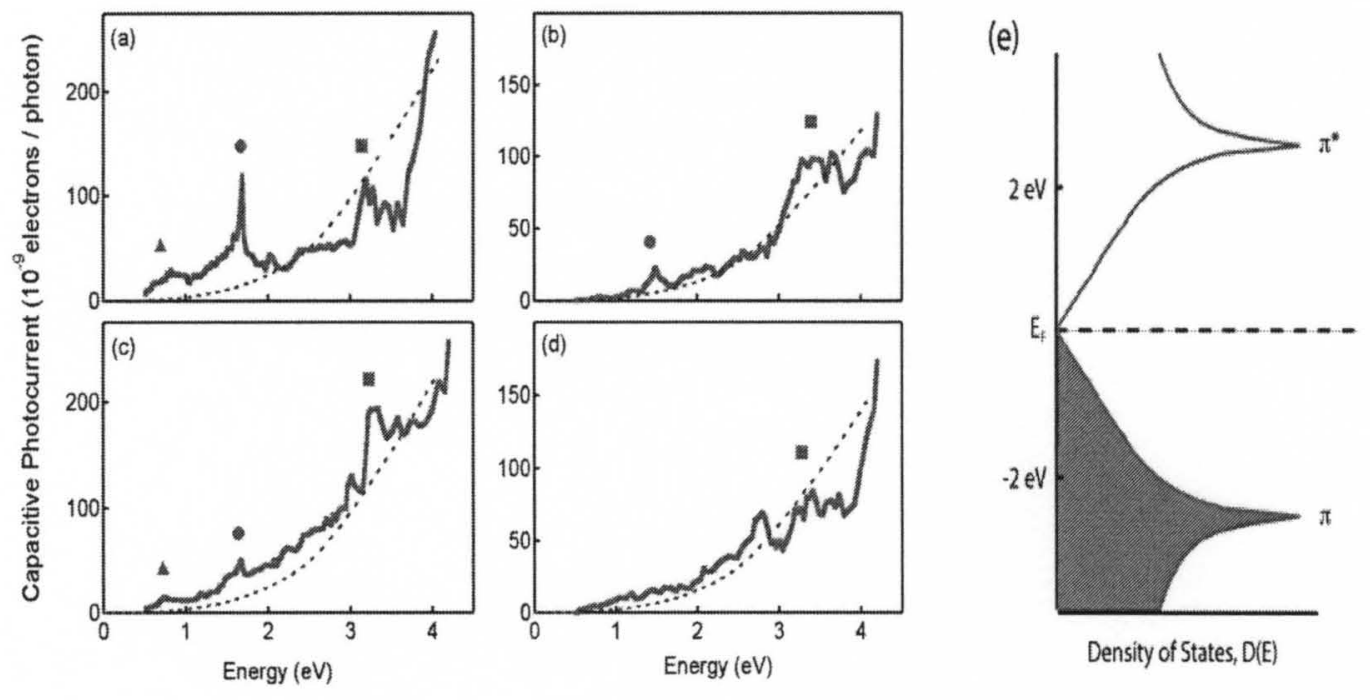

Figure 5.3. Capacitive photocurrent spectra as a function of excitation energy for four different individual graphene sheets. (a) \& (b) Graphene transferred using $10 \%$ aqueous solution of $\mathrm{HNO}_{3}$. (c) \& (d) Graphene transferred using ferric chloride solution. Samples ((a) and (c)) were measured using the standard measurement configuration shown in Figure 1(a), while the other two (b) and (d) were measured with the graphene lying on quartz, with a remote silver paste contact placed on a corner of the graphene sheet. Reproducible peaks near $0.7 \mathrm{eV}, 1.6 \mathrm{eV}$ and $3.2 \mathrm{eV}$ are marked with $\boldsymbol{\Delta}$, $\bullet$, and $\mathbf{m}$, respectively. The dashed lines show the calculated spectrum following equation (1) and using density of states, $D(E)$, of graphene as shown in (e). 
The data can be analyzed using the standard description for electron band-to-band transitions [76-77]. Assuming that all photo-generated electron-hole pairs separate under the applied dc bias to be detected as photocurrent, the capacitive photocurrent signal $C(h v)$ will be proportional to the product of the initial and final states integrated over all possible transitions for a given excitation energy $h v$, or

$$
C(h v)=\kappa \int_{E_{F}-h v}^{E_{F}} D(E) D(E+h v) d E
$$

where $D(E)$ is the density of states in the graphene, $E_{F}$ is the Fermi energy, and $\kappa$ is a proportionality constant. To simplify the analysis, we assume that the probability for all different possible transitions is equal ( $\kappa$ held constant), and that all states below the Fermi energy are filled, while states above the Fermi energy are empty (zero temperature approximation) [78-79].

The density of states for graphene (shown in Figure 5.3(e)) is calculated using the tight binding dispersion relation with an overlap integral of $2.6 \mathrm{eV}$ [80-82], and neglecting nearest neighbor hopping. The solution to equation (1) is then calculated numerically, adjusting kappa $(\kappa)$ to provide the best possible fit to the data in Figure 5.3(a)-(d). The absence of a gap in the semi-metallic graphene $D(E)$ means that the capacitive photocurrent increases monotonically as a function of excitation energy. This is in qualitative agreement with the general trend observed in the data; however, the fluctuations observed in the experiment are not reproduced. 
Capacitive photocurrent measurements were then performed on a series of GO samples. The results are shown in Figure 5.5 (blue solid lines). Two of the samples ((a) and (d)) were synthesized in our laboratory, using the procedure described in the experimental section below, while the other two samples ((b) and (c)) were purchased from a commercial supplier (Graphene Supermarket). The magnitude of the GO capacitive photocurrent signal is considerably smaller than that observed for the graphene, due to the reduced conductivity of the oxidized graphene. Three peaks are observed at approximately $0.7,1.6$ and $3.2 \mathrm{eV}$, which correlate with the peak positions observed in the graphene (marked as $\boldsymbol{\Delta}, \bullet$, and $\boldsymbol{\varpi}$ ). In contrast to the graphene results, however, no background increase in the photocurrent occurs with increasing excitation energy. In addition, there are strong minima in the photocurrent at $2.1 \mathrm{eV}$, and beyond 4 $\mathrm{eV}$, indicating gaps in the density of states. Similar results are observed for all four GO samples, although there are variations in the peak position and magnitude. Three of the samples ((a) (b), and (d)) were measured using the standard configuration shown in Figure 5.2(a), while sample (c) was measured with the GO lying on quartz, with a remote silver paste contact (as described above). The difference in substrate has little effect on the peak positions, showing that the peaks are not due to graphene / ITO interface states (as was suggested by the graphene data). 

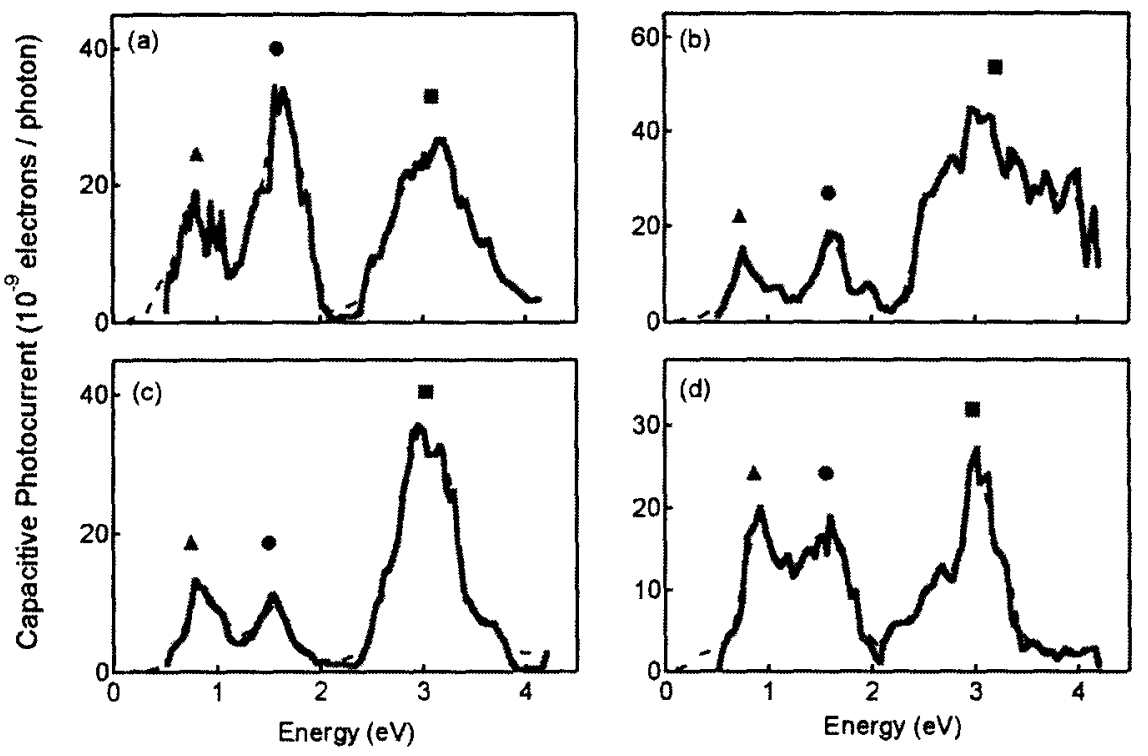

Figure 5.4. Capacitive photocurrent spectra of four GO samples (solid blue line). Three peaks are observed at $0.7,1.6$ and $3.2 \mathrm{eV}$, which correlate with the peak positions observed in the graphene (marked as $\boldsymbol{\Delta}, \bullet$, and $\mathbf{m}$, respectively). The dashed red curves are calculated following equation (1) and using the density of states for GO as shown in Figure 5.5.

For GO, the DOS is unknown, so it is not possible to calculate $C(h v)$ directly from equation (1). However, using the capacitive photocurrent spectrum and working backwards from equation (1), it is possible to determine a fit of $D(E)$ to the experimental data. The analysis is as follows (see supplementary information for a detailed description of the fitting procedure). The capacitive photocurrent $C_{i}$ is measured for a set of equally spaced excitation energies, $h v=i \times \Delta \mathrm{E}$, where $i$ is an integer ranging from $i_{\min }$ to $i_{\max }$, and $\Delta E$ is the excitation energy spacing. This leads to the following discretized version of equation (1):

$$
C_{i}=\kappa \sum_{j=1}^{i} D_{-j} D_{(i-j)+1}
$$


Here, $D_{ \pm j}$ is defined at energies $E_{+j}=E_{F}+(j-1 / 2) \Delta E$ for empty states above the Fermi energy, and $E_{-j}=E_{F}-(j-1 / 2) \Delta E$ for filled states below the Fermi energy for $j$ ranging from 1 to $i_{\max }$. This gives a set of $\left(i_{\max }-i_{\min }+1\right)$ coupled equations which together contain $2 \times i_{\max }$ different $D_{j}$ values. In practice, an interpolation is first done from the measured data so that in all cases $\Delta \mathrm{E}=0.11 \mathrm{eV}, i_{\min }=5$, and $i_{\max }=37$.

Since there are more than twice as many unknown $D_{j}$ values as there are equations, it is impossible to solve for $D_{j}$ exactly. Instead, a fitting procedure is used. All values of $D_{j}$ are initially set to an arbitrary constant. Next, one of the $D_{j}$ is chosen at random, and the Levenberg-Marquardt algorithm is used to determine the value for this $D_{j}$ that provides the best possible fit to the experimental measurements (keeping all other $D$ values fixed). This is repeated until a fit has been done for each $D_{j}$. The entire process is then repeated until a stable set of $D_{j}$ values has been obtained. This fitting algorithm is found to generate a reproducible and stable $D(E)$ versus energy dependence, from which a close approximation to the observed capacitive photocurrent spectrum can be obtained. 


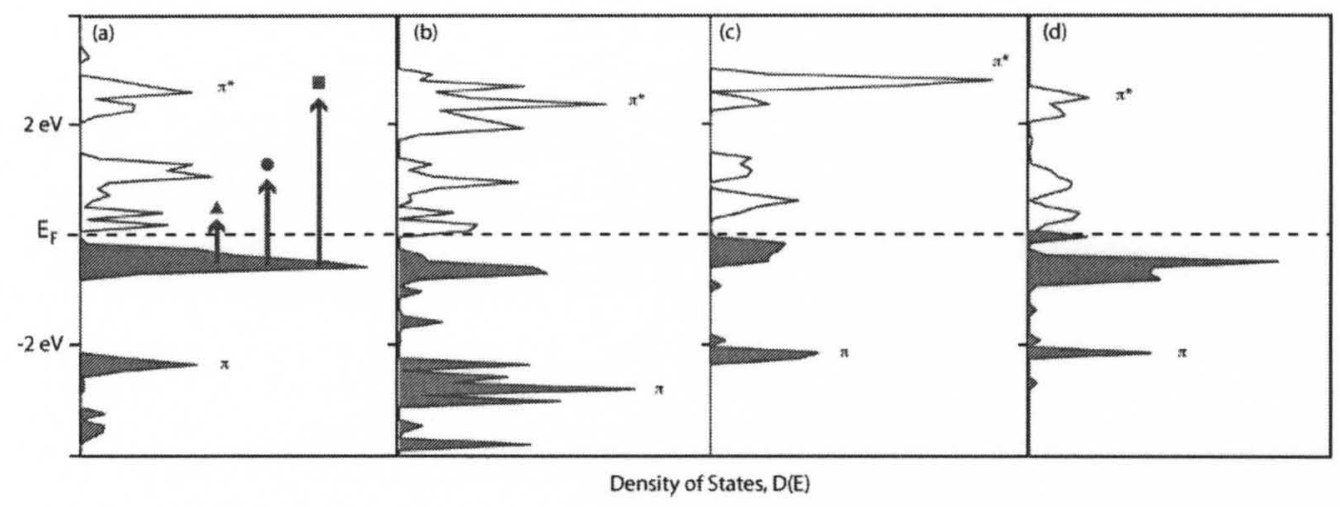

Figure 5.5. Predicted density of states for GO following the fitting of equation (2) to the data plotted in Figure 4. Transitions between the filled and empty states (as shown by the $\boldsymbol{\Delta}, \bullet$, and $\mathbf{m}$ transitions in (a)) produce the peaks observed in the capacitive photocurrent.

Figure 5.5 shows the GO DOS determined by applying this procedure to the four capacitive photocurrent spectra shown in Figure 4, while the dashed red lines in Figure 5.4 show the capacitive photocurrent calculated from the extracted GO DOS. (The excellent match between the experimental and theoretical capacitive photocurrent spectra demonstrates that the fitting procedure is working properly). There is a clear variation between the DOS calculated for the different samples, but each also contains a similar set of peaks which provide for the transitions observed in the photocurrent. There are two high energy peaks in the GO DOS near $+/-2.6 \mathrm{eV}$ which are approximately symmetric to the Fermi energy, and three mid-gap features, one occupied below the Fermi energy, and two empty above the Fermi energy. The high energy peaks at $+/-2.6 \mathrm{eV}$ correlate reasonably well with the $\pi$ and $\pi^{*}$ peaks observed in the graphene DOS, and are marked as such in the Figure. Their presence implies that some influence from the graphene still remains, even in the oxidized sample, and agrees with the picture that there is significant inhomogeneity of the oxidation coverage within the GO plane. Note that the $\pi$ and $\pi^{*}$ peaks are not expected to be precisely symmetric with respect to the Fermi energy 
because the sample can be doped by the interaction with the substrate or the environment. The amount of this doping varies from sample to sample.
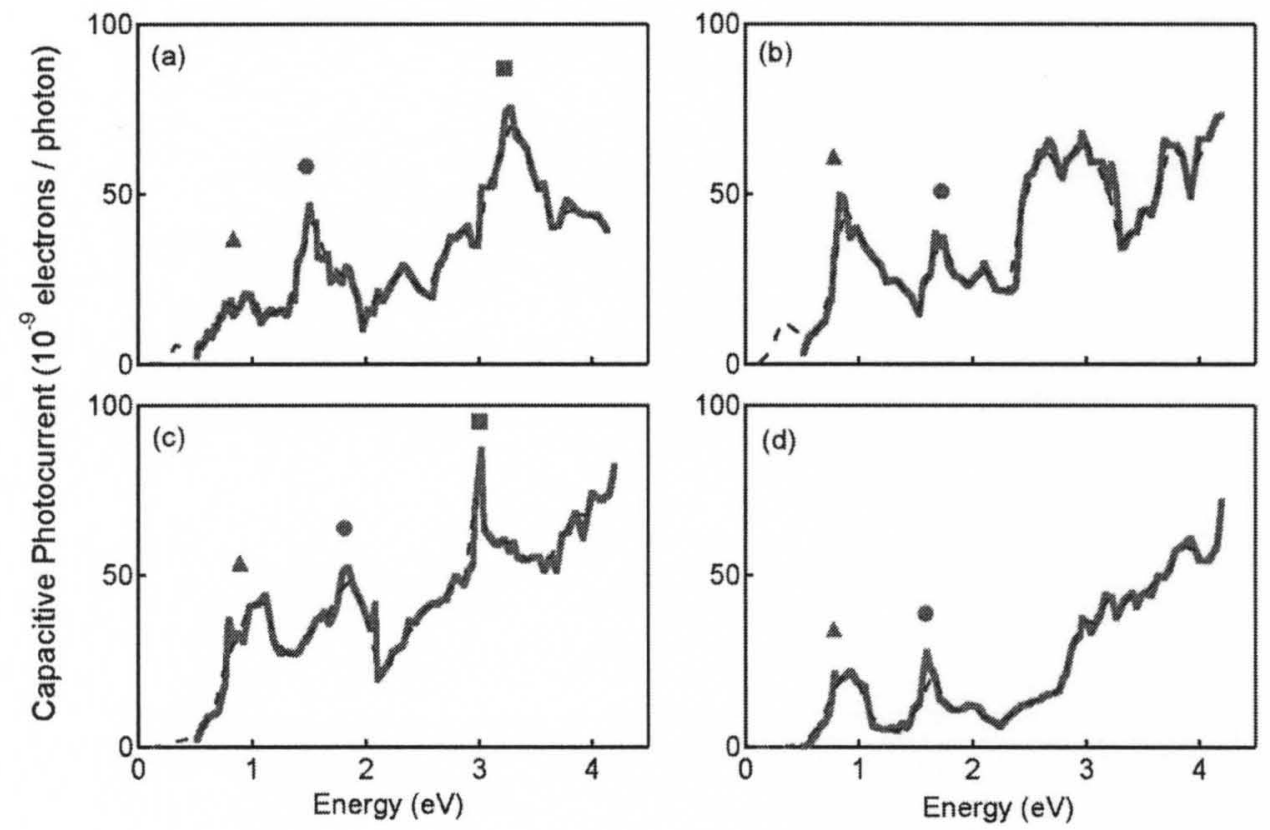

Figure 5.6. Experimental results of capacitive photocurrent spectroscopy on rGO (solid blue curves). Three peaks, on top of a monotonically increasing background, are observed at $0.7,1.6$ and $3.2 \mathrm{eV}$, (marked as $\boldsymbol{\Delta}$, $\bullet$, and $\mathbf{m}$, respectively). The dotted curve is calculated following equation (1) and using the density of states for $\mathrm{rGO}$ as shown in Figure 5.7.

Comparison of the capacitive photocurrent data to the calculated density of states shows that the three peaks in the photocurrent can be accounted for by transitions from the lowest energy occupied peak to the three lowest energy unoccupied peaks (marked as $\boldsymbol{\Delta}$, - and $\boldsymbol{\mathbf { a }}$ in Figure 5.5(a)). Two of these transitions compare favorably to photoluminescence measurements reported in the literature. The $\bullet-$ transition has energy of approximately $1.65 \mathrm{eV}$ (corresponding to wavelength of $750 \mathrm{~nm}$ ) which is equal to the peak luminescence energy emitted from a GO sample in solution. The $\mathbf{m}$-transition has energy of approximately $3.1 \mathrm{eV}$ corresponding to a wavelength of $400 \mathrm{~nm}$. This is very 
close to the wavelength of blue luminescence observed for fully reduced GO (390 nm). While these transitions match reasonably well, the luminescence peaks are very broad, and variations are observed due to the geometry of the GO sample, and the background pH. Clearly, additional mid-gap features (such as that producing the $\boldsymbol{\Delta}$ - transition) could occur due to the environment in contact with the GO, which can broaden, or shift the PL spectrum.

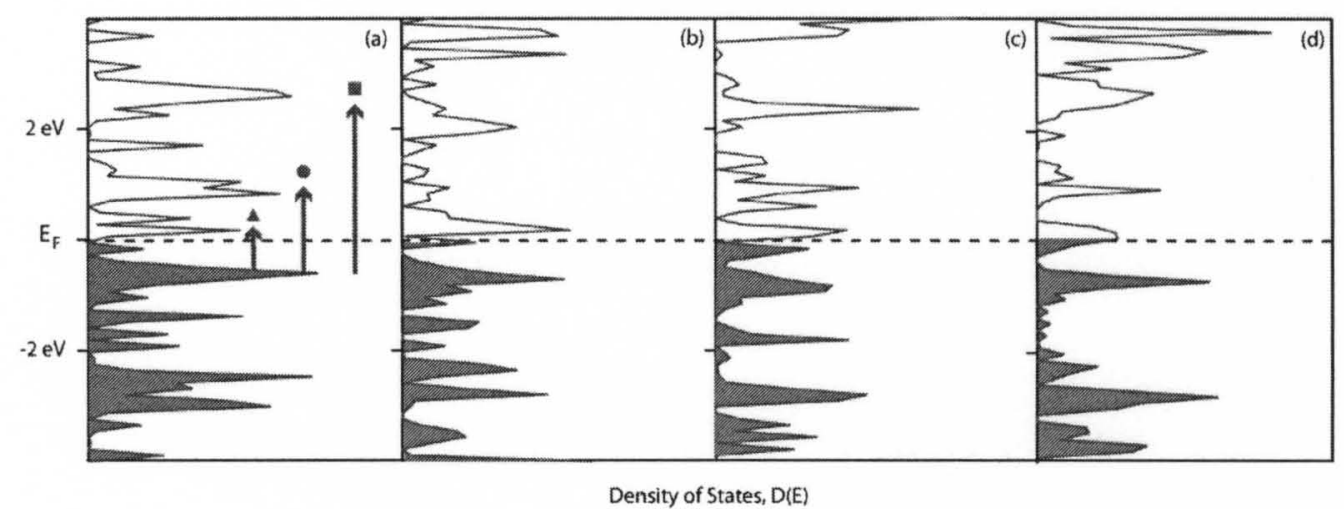

Figure 5.7. Predicted density of states for rGO following the fitting of equation (2) to the data plotted in Figure 5.6. Transitions between the filled and empty states (as shown by the $\boldsymbol{\Delta}$, $\bullet$, and $\boldsymbol{\|}$ transitions in (a)) produce the peaks observed in the capacitive photocurrent.

To see the effect of reduction on the GO density of states, capacitive photocurrent measurements were also performed on four different rGO samples. The results are plotted in Figure 5.6. As with the GO, two of the samples ((a) and (d)) were synthesized as mentioned earlier, while the other two samples ((b) and (c)) were purchased from a commercial supplier (Graphene Supermarket); three of the samples ((a) (b), and (d)) were measured using the standard configuration shown in Figure 5.1(a), while sample (c) was measured with the rGO lying on quartz, with a remote silver paste contact. The spectra are more complicated, and less similar to each other than with the GO and graphene samples. In general, the three peaks (marked as $\mathbf{\Lambda}, \bullet$, and $\mathbf{m}$ ) are once again observed, 
but they now lie on a gradually increasing background. Applying the described fitting procedure to this data, we extract the DOS plots shown in Figure 5.7. The peaks observed in the GO DOS are still visible, however, a number of additional peaks fill the hard gaps where $D(E)$ dropped to zero in the GO. As shown by the dashed line in Figure 5.6(a), the capacitive photocurrent calculated from this density of states provides a good fit to the measured result.

From these results it is clear that the GO reduction does not completely remove the influence of the oxidation and return the sample to a pristine state. This agrees with the XPS results above, where the presence of oxygen is still detected in the reduced sample. Also, it has been demonstrated that GO reduction does not completely quench the luminescence, and thus the recombination centers due to the oxygen must still be present. Recently, Gómez-Navarro et al. has shown that rGO consists of fully reduced graphene areas in a matrix of partially reduced graphene oxide with clustered defects. The presence of additional peaks in the DOS of rGO can be attributed to the combination of the DOS due to graphene and the energy states originating due to these clustered defects. Second, the energy of the states due to the oxygen does not change with reduced oxygen coverage. Instead, there is simply a decrease in the average electronic density at the peak energies. This implies that there is considerable inhomogeneity in the oxygen coverage, and that it cannot be assumed that the oxygen occupies the graphene surface in a few stable, well defined uniform configurations. 


\subsection{DETAILED DESCRIPTION OF THE FITTING PROCEDURE}

The experimental data consists of a set of capacitive photocurrent measurements $C_{i}$, and corresponding excitation energies $E_{i}$, where $i$ is an integer running from $i_{\min }$ to $i_{\max }$. The spacing between excitation energies $\Delta E$ is kept constant, so that $E_{i}=i \times \Delta E$, and $i_{\min }$ and $i_{\max }$ are defined by the minimum excitation energy $\left(i_{\min } \times \Delta E\right)$ and the maximum excitation energy $\left(i_{\max } \times \Delta E\right)$ available from the measured data. Using the same energy spacing $\Delta E$, a discretized version of the density of states, $D_{ \pm j}$ is defined at energies $E_{+j}=E_{F}+(j-1 / 2) \Delta E$ for empty states above the Fermi energy, and $E_{+j}=$ $E_{F}+(j-1 / 2) \Delta E$ for filled states below the Fermi energy. Figure 5.8 shows the discretized density of states $D_{ \pm j}$ plotted relative to the Fermi energy $E_{F}$, along with the measured capacitive photocurrent points $C_{i}$.

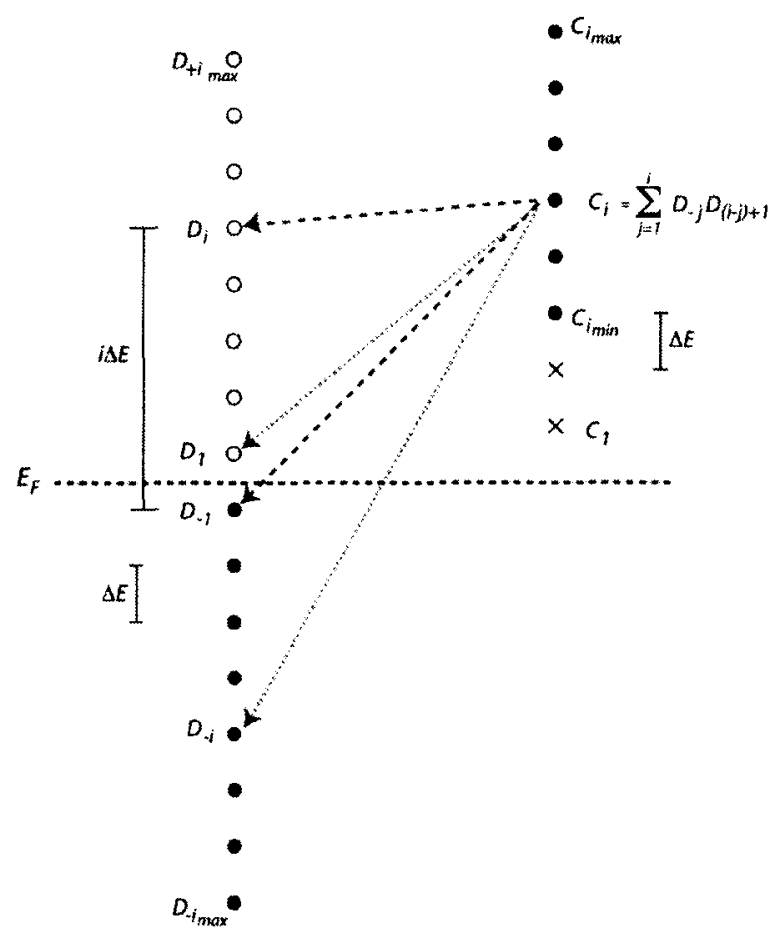

Figure 5.8. Diagram showing the discrete density of states, $D_{ \pm j}$, and the measured capacitance photocurrent $\mathrm{Ci}$. Points marked with an $\mathrm{x}$ are un-measured $\mathrm{C}_{\mathrm{i}}$ values below $\mathrm{C}_{\text {imin }}$ 
The model assumes that the capacitive photocurrent $C_{i}$ is generated by band-to-band transitions at the excitation energy i $\Delta E$ between the filled states below $E_{F}$ and the empty states above $E_{F}$. For example, a filled state at $E_{-j}$ will be excited by an energy $i \Delta E$ to an empty state at $E_{(i-j)+1}$, where $i \geq j$. The contribution of this transition to the capacitive photocurrent $C_{i}$ is proportional to the product of the associated density of states, $D_{-j} D_{i-}$ $\left.{ }_{j}\right)+1$. Taking the sum of the contributions from all possible transitions gives:

$$
C_{i}=\sum_{j=1}^{i} D_{-j} D_{(i-j)+1}
$$

Figure 5.8 shows the situation where $i=5$, and the excitation energy is $5 \Delta E$. In this case, the possible transitions extend over the region indicated by the dotted and dashed lines, or from

$D_{.5} D_{1}$ to $D_{-l} D_{5}$. In order for equation (3) to be defined for each measured $C_{i}$, the density of states $D_{j}$ must be defined for all $j$ ranging from -1 to $-i_{\max }$ and from 1 to $+i_{\max }$. This means that there are at least twice as many $D_{j}$ values as $C_{i}$ values. It is therefore impossible to solve for $D_{j}$ exactly, and a fitting procedure must instead be used.

The fitting routine was written using the programming capabilities of WaveMetrics Igor Pro software (version 6.02A). To help explain the procedure, the calculation of the DOS for the graphene oxide sample, (shown as Figure 5.4(a)) will be described step-bystep.

First, an interpolation is done from the measured data to obtain a series of equally spaced capacitive photocurrent values $C_{i}$. Figure 2 shows the measured capacitive photocurrent data (solid blue line) and the interpolated points $C_{i}$ (red dots). In the interpolated data, $\Delta \mathrm{E}=0.11 \mathrm{eV}, i_{\min }=5$, and $i_{\max }=37$. The number of $C_{i}$ values is reduced below the maximum number of measured values to minimize fluctuations in the fitting procedure. 


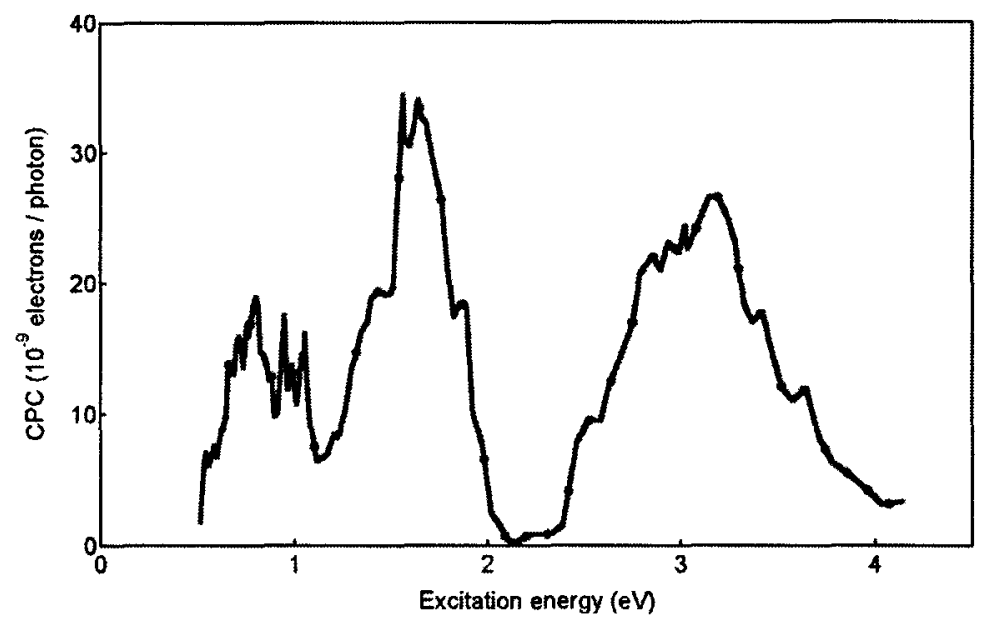

Figure 5.9 CPS of first device of GO, dots represent the interpolated points used for fitting procedure.

Next, all the $D_{j}$ values are set equal to an initial value (in this case, $D_{j}=0.2$ ). The initial value is chosen so that the magnitude of the photocurrent calculated from equation (1) is approximately equal to the measured values. Figure 5.10(a) shows the measured $C_{i}$ (solid blue line) and the calculated $C_{i}$ (dashed red line) as a function of excitation energy, while Figure $5.10(\mathrm{~b})$ shows $D_{j}$ as a function of energy. Not surprisingly, the constant DOS gives a very poor fit to the experiment.
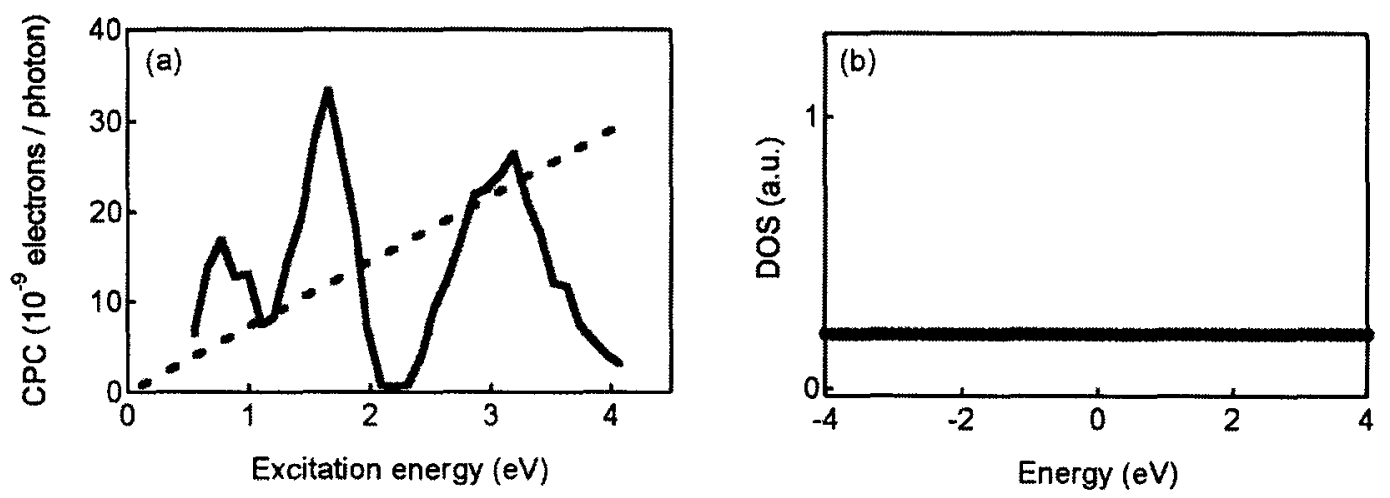

Figure 5.10 Calculated capacitive photocurrent for a constant DOS. 
Ideally, each $D_{j}$ value should be optimized simultaneously in equation (3) to give the best possible match to the $C_{i}$ values. However, this is difficult to do for such a large number of fitting parameters. Instead, the $D_{j}$ values are optimized one at a time, keeping all other values fixed. This process is repeated until a stable set of $D_{j}$ values is determined.
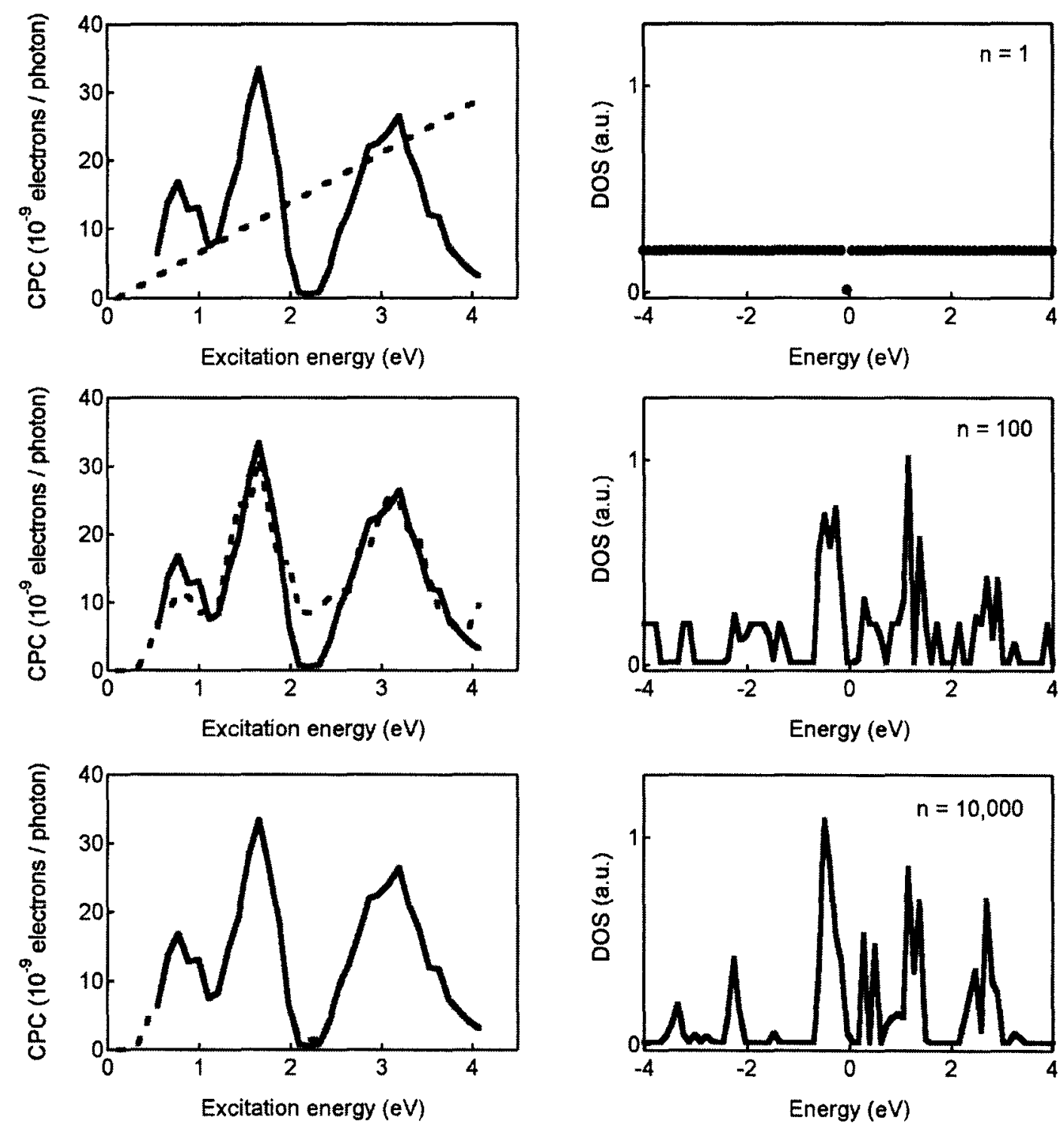

Figure 5.11 Demonstration of fitting procedure after number of runs $(n=1,100,10000)$. 
Figure 5.11 shows the results of this procedure. For $n=1$, a single $D_{j}$ value (in this case $j=-1$ ) is optimized to provide the best possible fit to the data, which is still not very good. Repeating up to $n=100$, and the fit starts to resemble the data, while a well-defined structure starts to appear in the DOS. Repeating up to $n=10,000$, and the fit accurately matches the data. The calculated $\mathrm{Dj}$ values are now stably determined and no longer change with continued optimization.
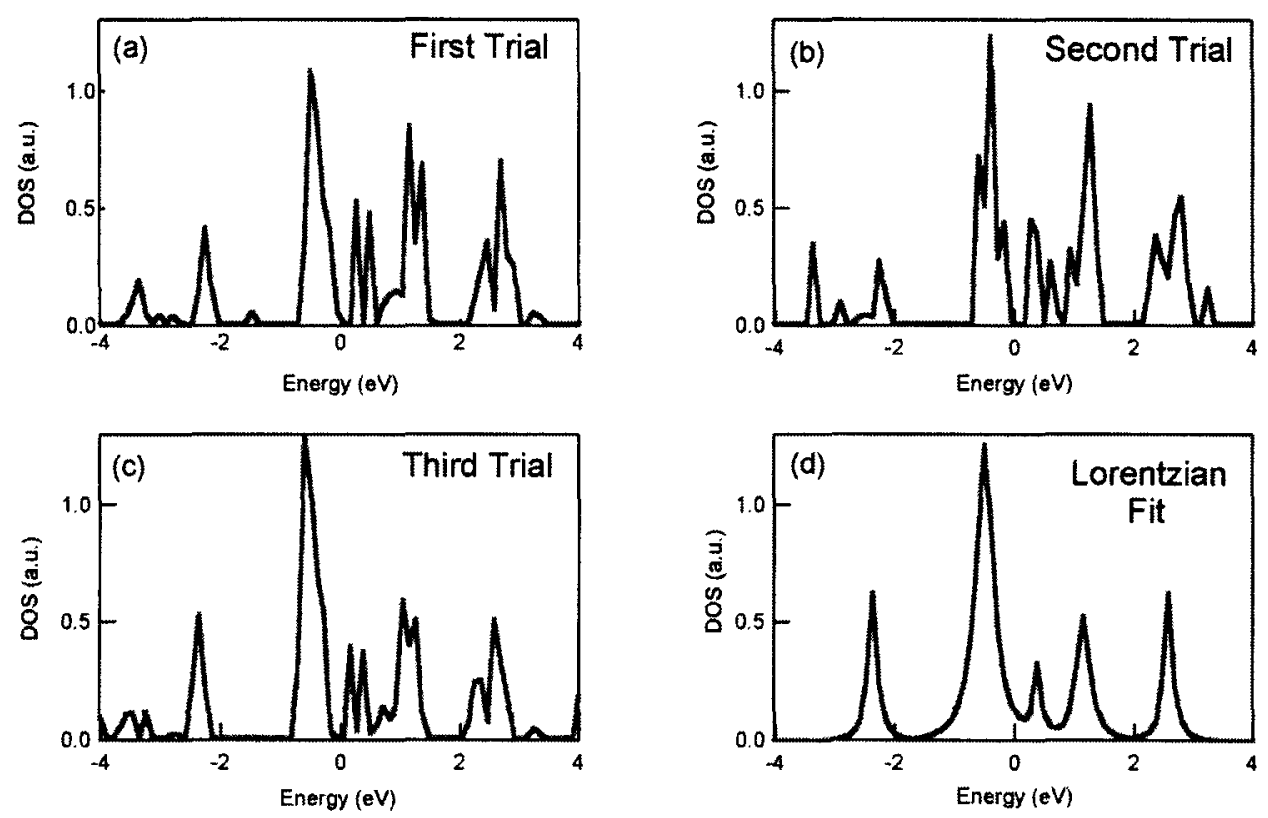

Figure 5.12 Three calculated DOS curves for one GO device. (d) Shows the Lorentzian fit to the peaks seen in calculated DOS.

Because the DOS is not uniquely determined by the data, starting with a new set of $\mathrm{Dj}$ values and repeating the fitting produces a slightly different result for the DOS. Figure 5 (a)-(c) shows 3 different trials of the fitting procedure. In each case, the fit produces a somewhat different stable set of Dj values, however (as shown in Figure 5.13), the CPC calculated from each of the trials is the same. This shows that the fitting procedure does not produce a unique solution for the DOS, but only one possible solution that matches with the data. 


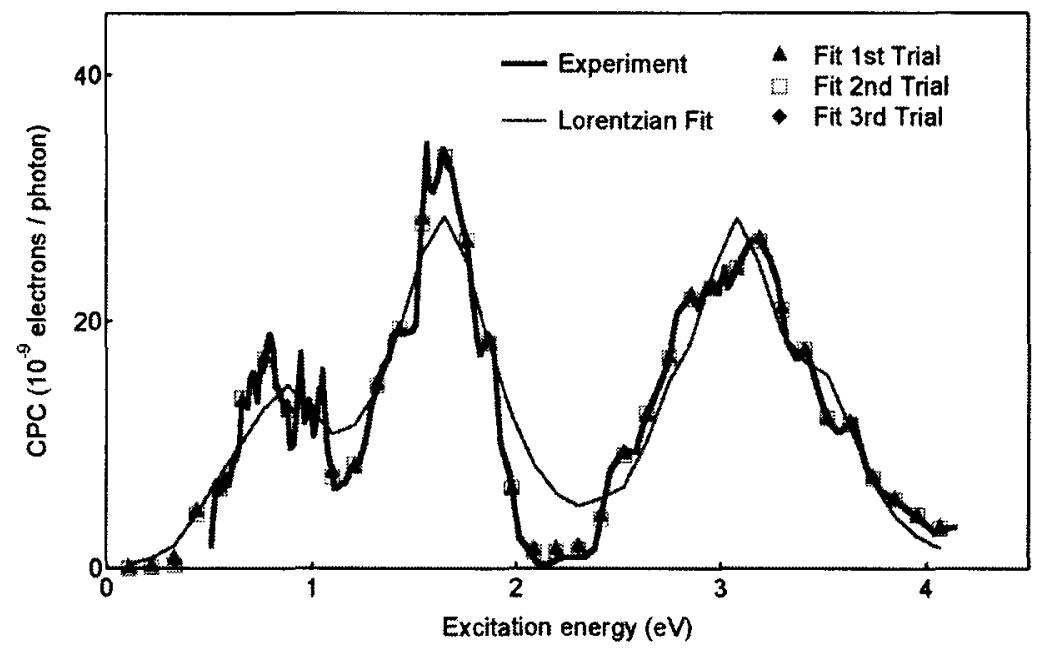

Figure 5.13 Fitting procedure used for the DOS curves shown in Figure 5.12.

Despite these variations, the basic structure of the DOS is stable from trial to trial, and among different GO samples. Five main peaks appear: two associated with the graphene $\pi$ and $\pi^{*}$ peaks and three more produced by the oxidation. A combination of a Lorentzian fit to each of these peaks gives the DOS shown in Figure 5.12(d). While not exact, the CPC calculated from this combination of Lorentzians, also provides reasonably good fit to the data (see solid red line in Figure 5.13). 


\section{CHAPTER VI}

\section{CONCLUSIONS AND FUTURE DIRECTIONS}

\subsection{CONCLUSIONS}

A direct experimental probe of the GO density of states as a function of oxygen coverage using capacitive photocurrent spectroscopy is provided. A novel capacitive photocurrent spectroscopy technique is used to calculate the density of states (DOS) of numerous GO and rGO devices. Three intense additional peaks (separated by hard gaps) in the DOS of GO appear near the Fermi energy (attributed to the presence of oxygen functional groups) along with standard $\pi / \pi^{*}$ peaks present in graphene DOS. This confirms the theory prediction of multi-gap DOS for GO. Reduced graphene oxide (rGO) shows a distribution of peaks for the entire energy range probed in the experiment. Three similar peaks (less intense as compared to peaks seen in GO) with no hard gaps near Fermi energy were seen for the rGO sample providing the evidence of some residual oxidation. Extra peaks seen in rGO sample are attributed to defect creation during the reduction process. XPS measurements confirm the presence of similar oxygen functional groups in all the samples (CVD grown graphene, GO and rGO) with varying intensity dictating the oxidation content percentage. 


\subsection{FUTURE DIRECTIONS}

As shown in the previous chapter the capacitive photocurrent measurements and the density of state calculation procedure can be employed to predict the DOS for new nanomaterials (including hydrogenated and fluorinated-graphene). The combination of CPS measurements and calculation procedure opens the door for predicting electrical and optical properties of new materials. CPS can be used as a optical characterization technique. 


\section{REFERENCES}

[1] G. Timp, "Nanotechnology." Springer, 1999.

[2] M. A. Reed, W. P. Kirk, "Nanostructures and Mesoscopic Systems." Academic Press, 1991.

[3] J. Toon, "Extending Moore's Law: Epitaxial Graphene Shows Promise for Replacing Silicon in High-Performance Electronics." Greorgia-Tech research news.

[4] R. Murali, Y. Yang, K. Brenner, T. Beck, J. D. Meindl, "Breakdown current density of graphene nanoribbons." Applied Physics Letters 94, 243114, 2009.

[5] T. Ando, Y. Arakawa, K. Furuya, S. Komiyama, H. Nakashima, "Mesoscopic Physics and Electronics." Springer, 1998.

[6] T. Chakraborthy, "Quantum Dots." Elsevier, 1999.

[7] S. Datta, "Electronic Transport in Mesoscopic Systems." Cambridge Studies in Semiconductor Physics and Micro-electronics Engineering, 1995.

[8] K. S Novoselov, A. K Geim, S. V. Morozov, D. Jiang, Y. Zhang, S. V. Dubonos, I. V. Grigorieva, A. A. Firsov, "Electric Field Effect in Atomically Thin Carbon Films." Science 2004, 306, 666-669.

[9] K.I. Bolotin, K.J. Sikes, Z. Jiang, M. Klima, G. Fudenberg, J. Hone, P. Kim, H.L. Stormer, "Ultrahigh electron mobility in suspended graphene." Solid State Communications 146 (2008) 351-355.

[10] L. A. Jaureguia, Y. Yue, A. N. Sidorov, J. Hu, Q. Yu, G. Lopez, R. Jalilian, D. K. Benjamin, D. A. Delk, W. Wu, Z. Liu, X. Wang, Z. Jiang, X. Ruan, J. Bao, S. S. Pei, Y. P. Chen, "Thermal Transport in Graphene Nanostructures: Experiments and Simulations." ECS Transactions, Volume 28, Issue 5, 2010.

[11] Y. M. Lin, C. Dimitrakopoulos, K. A. Jenkins, D. B. Framer, H. Y. Chiu, A. Grill, $\mathrm{Ph}$. Avouris, "100-GHz Transistors from Wafer-Scale Epitaxial Graphene." Science, Vol. 327 no. 5966 p. 662, 2010. 
[12] S. Y. Zhou, G. -H. Gweon, A. V. Fedorov, P. N. First, W. A. De Heer, D. -H. Lee, F. Guinea, A. H. C. Neto, A. Laranza." Substrate-induced bandgap opening in epitaxial graphene." Nature Materials, vol. 6, 2007.

[13] F. Xia, D. B. Farmer, Y.-M. Lin, Ph. Avouris, "Graphene Field-Effect Transistors with High On/Off Current Ratio and Large Transport Band Gap at Room Temperature." Nano letters, 10, 715-718, 2010.

[14] M. Y. Han, B. Özyilmaz, Y. Zhang, P. Kim, "Energy Band-Gap Engineering of Graphene Nanoribbons." PRL 98, $206805,2007$.

[15] A. N. Sidorov, T. Bansal, P. J. Ouseph, G. U. Sumanasekera, "Graphene nanoribbons exfoliated from graphite surface dislocation bands by electrostatic force." Nanotechnology 21, 2010.

[16] X. Li, X. Wang, L. Zhang, S. Lee, H. Dai, "Chemically Derived, Ultra smooth Graphene Nanoribbon Semiconductors." Science, vol. 319, 2008.

[17] D. R. Dreyer, S. Park, C. W. Bielawski, R. S. Ruoff, "The chemistry of graphene oxide." Chem. Soc. Rev., 39, 228-240, 2010.

[18] I. Gierz, C. Riedl, U. Starke, C. R. Ast, K. Kern, "Atomic Hole Doping of Graphene." Nano Lett., 8, 4603-4607, 2008.

[19] D. W. Boukhvalov, M. I. J. Katsnelson, "Modeling of Graphite Oxide." AM. CHEM. SOC., 130, 10697-10701, 2008.

[20] R. J. W. E. Lahaye, H. K. Jeong, C. Y. Park, Y. H. Lee, "Density Functional Theory Study of Graphite Oxide for Different Oxidation Levels." Phys. Rev. B, $79,125435,2009$.

[21] X. Wu, M. Sprinkle, F. Ming, C. Berger, W. A. De Heer, "Epitaxial graphene/graphene-oxide Junction: An essential step towards epitaxial graphene electronics." Phys. Rev. Lett., 101, 026801, 2008.

[22] Ph. Avouris, "Graphene: electronic and Photonic properties and devices." Nano letters, 10, 4285-4294, 2010.

[23] R. R. Nair, P. Blake, A. N. Grigorenko, K. S. Novoselov, T. J. Booth, T. Stauber, N. M. R. Peres, A. K. Geim, "Fine Structure Constant Defines Visual Transparency of Graphene." Science, vol 320, 2008.

[24] R. R. Nair, W. C. Ren, R. Jalil, I. Riaz, V. G. Kravets, L. Britnell, P. Blake, F. Schedin, A. S. Mayorov, S. Yuan, M. I. Katsnelson, H. M. Cheng, W. Strupinski, L. G. Bulusheva, A. V. Okotrub, I. V. Grigorieva, A. N. Grigorenko, K. S. 
Novoselov, A. K. Geim, "Fluorographene: a Two Dimensional Counterpart of Teflon.” Small, No. 24, 2877-2884, 2010.

[25] G. Eda, Y. Lin, C. Mattevi, H. Yamaguchi, H. Chen, I. Chen, C. Chen, M. Chhowalla, "Blue Photoluminescence from chemically derived grapehen oxide." Adv. Mater, 22, 505-509, 2010.

[26] Z. Luo, Y. Lu, L. A. Somers, A. T. C. Johnson, "High Yield Preparation of Macroscopic Graphene Oxide Membranes.” J. Am. Chem. Soc., 131, 898, 2009.

[27] X. Sun, Z. Liu, K. Welsher, J. T. Robinson, A. Goodwin, S. Zaric, H. Dai, "NanoGraphene Oxide for cellular Imaging and drug delivery." Nano Res, 1, 203-212, 2008.

[28] Z. Luo, P. M. Vora, E. J. Mele, A. T. C. Johnson, J. M. Kikkawa, "Photoluminescence and Band Gap Modulation in Graphene Oxide." Appl. Phys. Lett., 94, $111909,2009$.

[29] C. Hamilton, A. Barron, "Graphene." Connexions web site, http://cnx.org/content/m29187/1.3/, 2010.

[30] F. Rana, "Energy bands in the graphene: Tight binding and nearly free electron approach." http://courses.cit.cornell.edu/ece407/Lectures/handout 1 1.pdf, 2009.

[31] http://invsee.asu.edu/nmodules/carbonmod/pibond.html.

[32] J.-C. Charlier, P. C. Eklund, J. Zhu, A. C. Ferrari, "Electron and phonon properties of graphene: their relationship with carbon nanotubes." Carbon Nanotubes: Advanced Topics in the Synthesis, Structure, Properties and Applications. Topics in Applied Physics 111. Springer-Verlag, pp. 673-709. 2008.

[33] A. K. Geim, K. S. Novoselov, "The rise of Graphene." Nature Materials, vol 6, 2007.

[34] A. H. C. Neto, F. Guinea, N. M. R. Peres, K. S. Novoselov, A. K. Geim, "The electronic properties of graphene." Review of Modern Physics, vol 81, 2009.

[35] J. S. Bunch, "Mechanical and Electrical Properties of Graphene Sheets." Dissertation, Cornell University, 2008.

[36] R. Saito, G. Dresselhaus, M. S. Dresselhaus, "Physical Properties of Carbon Nanotubes." Imperial college press, 1998.

[37] M. S. Silberberg, "Chemistry : The molecular nature of Mater and change." McGraw-Hill, 2006. 
[38] C. C. -Tannoudji, B. Diu, F. Laloe, “ Quantum Mechanics VOL -1.” Hermann and John Wiley and sons Inc., 1997.

[39] H. O. Pierson, "Handbook of Carbon, Graphite, Diamond and Fullerenes: Properties, processing and applications." Noyes Publications, 1993.

[40] http://en.wikipedia.org/wiki/Orbital hybridisation.

[41] S. Datta, "Quantum Transport: Atom to Transistor." Cambridge University Press, 2005.

[42] http://en.wikipedia.org/wiki/Brillouin zone

[43] M. Wilson, "Electrons in Atomically thin carbon Sheets Behave like Massless Particles.” Physics Today, 2006.

[44] A. N. Sidorov, "Novel Techniques to produce and deposit N-layer Graphene : Their Physical Properties." Dissertation, University of Louisville, 2009.

[45] L.M. Malard, M.A. Pimenta, G. Dresselhaus, M.S. Dresselhaus, "Raman Spectroscopy in Graphene.” Physics Reports, 2009.

[46] N. Moral, "Production and Characterization of Carbon-based Nanomaterials: Single and double layer graphene for high pressure Raman spectroscopy." Thesis, Luleå University of Technology, 2009.

[47] M. S. Dresselhaus, "From Graphene to Graphite to Nanotubes to Graphene." APS March Meeting Graphene Tutorial, 2008.

[48] J. Maultzsch, S. Reich, C. Thomsen, H. Requardt, and P. Ordejón, "Phonon Dispersion in Graphite." Physical Review Letters, vol. 92, 2004.

[49] X. Lu, H. Huang, N. Nemchuk, R. S. Ruoff, "Patterning of highly oriented pyrolytic graphite by oxygen plasma etching." Applied Physics Letters, vol. 75, 1999.

[50] Y. Zhang, J. P. Small, W. V. Pontius, Philip Kim, "Fabrication and electric-fielddependent transport measurements of mesoscopic graphite devices." Applied Physics Letters, vol. 86, 2005.

[51] K. S. Novoselov, D. Jiang, F. Schedin, T. J. Booth, V. V. Khotkevich, S. V. Morozov, A. K. Geim, "Two-dimensional atomic crystals." PNAS, vol. 102, 2005.

[52] A Rigorous Introduction to Graphite, http://www.asbury.com/, Asbury Carbon. 
[53] M. J. McAllister,J.-L. Li, D. H. Adamson, H. C. Schniepp, A. A. Abdala, J. Liu, M. H.-Alonso, D. L. Milius, R. Car, R. K. Prud'homme, I. A. Aksay, "Single Sheet Functionalized Graphene by Oxidation and Thermal Expansion of Graphite." Chem. Mater., 19, 4396-4404, 2007.

[54] S. Stankovich, D. A. Dikin, R. D. Piner, K. A. Kohlhaas, A. Kleinhammes, Y. Jia, Y. Wu, S. T. Nguyen, R. S. Ruoff, "Synthesis of graphene-based nanosheets via chemical reduction of exfoliated graphite oxide." Carbon, 45, 1558-1565, 2007.

[55] Y. Zhu, S. Murali, M. D. Stoller, A. Velamakanni, R. D. Piner, R. S. Ruoff, "Microwave assisted exfoliation and reduction of graphite oxide for ultracapacitors." Carbon, 2010.

[56] W. A. de Heer, C. Berger, X. Wu, P. N. First, E. H. Conrad, X. Li, T. Li, M. Sprinkle, J. Hass, M. L. Sadowski, M. Potemski, G. Martinez, "Epitaxial graphene." Solid-State Communications, 2007.

[57] X. Li, W. Cai, J. An, S. Kim, J. Nah, D. Yang, R. Piner, A. Velamakanni, I. Jung, E. Tutuc, S. K. Banerjee, L. Colombo, R. S. Ruoff, "Large- area Synthesis of High-Quality and uniform Graphene Films on copper Foils." Science, vol 324, 2009.

[58] M. Borysiak, "Graphene Synthesis by CVD on Copper Substrates." NNIN REU Research Accomplishments, 2009.

[59] Y. Zhu, S. Murali, W. Cai , X. Li, J. W. Suk, J. R. Potts, R. S. Ruoff, "Graphene and Graphene Oxide: Synthesis, Properties, and Applications." Adv. Mater., 22, 3906-3924, 2010.

[60] W. S. Hummers, R. E. Offeman, "Preparation of Graphitic Oxide." J. Am. Chem. Soc., 80, 1339, 1958.

[61] N. I. Kovtyukhova, P. J. Ollivier, B. R. Martin, T. E. Mallouk, S. A. Chizhik, B. V. Buzaneva, A. D. Gorchinskiy, "Layer-by-Layer Assembly of Ultrathin Composite Films from Micron-Sized Graphite Oxide Sheets and Polycations." Chem. Mater., 11, 771-778. 1999.

[62] W. Gao, L. B. Alemany, L. Ci, P. M. Ajayan, « New Insights into the Structure and Reduction of Graphite Oxide." Nature Chemistry, 1, 403-408, 2009.

[63] C. Gómez-Navarro, J. C. Meyer, R. S. Sundaram, A. Chuvilin, S. Kurasch, M. Burghard, K. Kern, U. Kaiser, "Atomic Structure of reduced graphene oxide." Nano letters, 10, 1144-1148, 2010.

[64] Z. Chen, Y. -M. Lin, M. J. Rooks, Ph. Avouris, "Graphene nano-ribbon electronics." Physica E, 40, 228-232, 2007. 
[65] I. Jung, D. A. Dikin, R. D. Piner, Rodney S. Ruoff, "Tunable Electrical Conductivity of Individual Graphene Oxide Sheets Reduced at Low Temperatures." Nano letters, 2008.

[66] C. Gómez-Navarro, R. T. Weitz, A. M. Bittner, M. Scolari, A. Mews, M. Burghard, K. Kern, "Electronic Transport Properties of Individual Chemically Reduced Graphene Oxide Sheets." Nano letts, vol 7, No. 11, 3499 -3503, 2007.

[67] M. S. Dresselhaus, G. Dresselhaus, R. Saito, A. Jorio, "Raman Spectroscopy of Carbon Nanotubes." Physics Reports, 2004.

[68] Z. Luo, T. Yu, Z. Ni, S. Lim, H. Hu, J. Shang, L. Liu, Z. Shen, J. Lin, "Electronic Structures and Structural Evolution of Hydrogenated Graphene Probed by Raman Spectroscopy." The journal of Physical Chemistry C, 115, 1422-1427, 2011.

[69] A. Gupta, G. Chen, P. Joshi, S. Tadigadapa, P.C. Eklund, "Raman Scattering from High-Frequency Phonons in Supported n-Graphene Layer Films." Nano letts., vol. 6, No. 12, 2667-2673, 2006.

[70] A. D. Mohite, "Optical characterization of carbon nanotubes using the displacement photocurrent technique." Dissertation, University of Louisville, 2007.

[71] M. E. Fermann, A. Galvanauskas, G. Sucha, "Ultrafast Lasers: Technology and Applications." Marcel Dekker Inc., 2003.

[72] 'Hurricane", User's manual, Spectra-physics Lasers.

[73] “Mai-Tai”, User's manual, Spectra-physics Lasers.

[74] B. J. Clark, T. Frost, M. A. Russell, "UV Spectroscopy Techniques, Instrumentation, Data Handling." Chapman and Hall,1993.

[75] A. D. Mohite, S. Chakraborty, P. Gopinath, G. U. Sumanasekera, B. W. Alphenaar, "Displacement Current Detection of Photoconduction in Carbon Nanotubes." Appl. Phys. Lett., 86, 061114, 2005.

[76] A. D. Mohite, T. S. Santos, J. S. Moodera, B. W. Alphenaar, "Observation of the Triplet Exciton in EuS-Coated Single-Walled Nanotubes." Nature Nanotechnology, 4, 425-429, 2009.

[77] R. J. Waltman, J. Pacansky, C. W. Bates, "X-ray Photoelectron Spectroscopic Studies on Organic Photoconductors: Evaluation of Atomic Charges on Chlorodiane Blue and p-(Diethylamino)Benzaldehyde Diphenylhydrazone." Chem. Mater., 5, 1799-1804, 1993. 
[78] Y. Bouizem, A. Belfedal, J. D. Sib, L. Chahed, "Density of States in Hydrogenated Amorphous Germanium Seen via Optical Absorption Spectra." Solid State Communications, 126, 675-680, 2003.

[79] R. Bouzerar, A. Zeinert, H. J. Von. Bardeleben, "Correlation of Optical Absorption and Density of Paramagnetic Centers in a-C:H Films." Diamond \& Related Materials, 14, 1108-1111, 2005.

[80] S. Reich, J. Maultzsch, C. Thomsen, "Tight-binding Description of Graphene." Phys. Rev. B, 66, 035412, 2002.

[81] J. C. Charlier, X. Gonze, J. P. Michenaud, "First-Principles Study of the Electronic Properties of Graphite.” Phys. Rev. B, 43, 4579-4589, 1991.

[82] A. Grüneis, C. Attaccalite, L. Wirtz, H. Shiozawa, R. Saito, T. Pichler, A. Rubio, "Tight-binding Description of the Quasiparticle Dispersion of Graphite and FewLayer Graphene." Phys. Rev. B, 78, 205425, 2008. 


\section{CURRICULUM VITAE}

\section{TANESH BANSAL}

528 Atwood Street, Apt \#1,

(502) 8761219

Louisville, KY - 40217

tanesh@gmail.com

\section{RESEARCH INTERESTS}

Nano-electronics, Spintronics, Micro/nano-fabrication, Synthesis and characterization (electrical, magnetic and optical) of nano-materials, Renewable energy research with emphasis on photo-voltaic devices, Flexible interconnects, electrodes and sensors, Carbon electronics and optoelectronics, Low temperature device physics via Coulomb blockade and transport properties under high magnetic fields.

\section{PROFESSIONAL PROFILE}

- Doctoral candidate in Nanoscale Device Research Lab (NDRL) under Dr. Bruce W. Alphenaar. (Graduating in May2011)

- 6+ years of hands on experience in applied and fundamental research in the field of Micro/Nano-scale device fabrication, Magneto-electronic transport, Optical characterization, Material Science, Semi-conductor Physics, Cryogenics and Vacuum technologies.

- Proven research track with 6 peer reviewed journal papers, 8 Oral and 4 poster presentations at various international conferences (APS, IEEE-Nano, EPSCoR). 
- Recognized for research in the field of spintronics in "Science Editors' choice Highlights of the recent literature 2006" and received an invitation from IEEENano06, Cincinnati, OH to present the research work as an "Invited Talk".

- Supervision and training of M.S. and new Ph.D. students for e-beam lithography, ebeam evaporation, AFM and Spectra Physics femto-second pulse laser system with tunable OPA capabilities.

- Proven ability to work both independently and as a part of multi-disciplinary researchers team.

\section{EDUCATION}

Ph.D. Electrical Engineering (May 2007- Present)

University of Louisville, Louisville, KY under Dr. Bruce W. Alphenaar

M.S. Electrical Engineering (Aug 2003 - May 2007)

University of Louisville, Louisville, KY under Dr. Bruce W. Alphenaar

B. Tech. Electronics and Communication Engineering (Aug1998 - June 2002)

Kurukshetra University, Haryana, INDLA

\section{TECHNICAL EXPERTISE}

- Expertise in Clean Room routines, micro/nano-scale device fabrication processes

○ Lithography: Optical lithography, AB-M Mask aligner (Split field alignment) and E-beam Lithography. 
- Etching: Reactive ion etching, Wet etching (experience in handling HF, Nanostrip).

- Thin film Deposition: RF/DC sputter, Thermal / E-beam evaporation, Spin coating of Photo-voltaic polymers.

- Wire Bonding: Ball bonder.

- Chemical Vapor Deposition (CVD): synthesis of Graphene monolayer and Single wall carbon nanotubes.

- Material characterization: SEM, AFM, XRD, Energy Dispersive Spectroscopy (EDS), UV-Vis NIR, Raman Spectroscopy, X-ray Photoelectron Spectroscopy (XPS), Capacitive Photocurrent Spectroscopy (CPS).

- Expertise in handling Cryogenic liquids and systems, Vacuum systems, Electro- / superconducting magnets.

- Spectra Physics femto-second pulsed Laser; Hurricane (MaiTai, Evolution, Regenerative Amplifier) pumped OPA-800 CF.

- Experienced in troubleshooting of equipment problems and Labview programs for data acquisition.

- Software Packages: LabView, Matlab, L-edit, DesignCad, IGOR, Peakfit, Origin.

\section{PROFESSIONAL EXPERIENCE}

\section{GRADUATE RESEARCH ASSISTANT, University of Louisville}

(May 2004 - Present)

Carried out competitive research in the areas listed below: 


\section{Graphene Opto-electronics and Spectroscopy}

- First experimental evidence provided for the multi-energy band gap configuration of Graphene Oxide (GO) density of states (DOS).

- Developed a new method to characterize nano-materials (Graphene, SWNTS) using Capacitive Photocurrent spectroscopy.

- Studied the difference in the vibrational energy spectrum of graphene, graphene oxide (GO) and reduced graphene oxide (RGO) using Raman Spectroscopy.

- Performed X-ray Photoelectron spectroscopy measurements to distinguish between the carbon spectrum of graphene, GO and RGO.

\section{Organic Photo-voltaic (solar cell)}

- First experimental evidence indicating decrease in photo-voltage of a BHJs solar cell simply by increasing the intensity of a narrow band of light wavelengths without altering background illumination.

- The bleaching results counter-intuitively suggest that the efficiency of these solar cells can be improved by filtering out light over a narrow band of wavelengths.

- Capacitive photocurrent spectroscopy reveals that the charge dissociation from the PCBM exciton is large despite the small photo-voltage signal.

\section{Device Physics \& Electron Transport}

- Performed low temperature $(4.2 \mathrm{~K}, 77 \mathrm{~K})$ magneto-electrical measurements on short channel ( $\sim 10 \mathrm{~nm}-20 \mathrm{~nm})$ nano-devices for Coulomb blockade and transport properties. 
- Fabricated individual single wall carbon nanotubes and monolayer graphene devices using e-beam and optical lithography.

- Electrical characterization of graphene devices (before and after the acid treatment forming graphene oxide).

- CVD synthesis of Graphene and Single wall carbon nanotubes on $\mathrm{Si} / \mathrm{SiO}_{2}$ and quartz substrates.

\section{Spintronics (carbon nanotubes)}

- Fabricated and demonstrated gate tunable $(+15 \%$ to $-10 \%)$ change in magnetoresistance of Ni contacted SWNT spin FET. (First evidence).

- Extended a new technique (Shadow Evaporation) for fabricating ferro-magnetically contacted (Ni) short channel $(\sim 10 \mathrm{~nm})$ single wall carbon nanotube (SWNT) spin FET.

- Conducted experiments on magnetic switching in Nickel contacted metallic and semiconducting single wall nanotubes at $4.2 \mathrm{~K}, 77 \mathrm{~K}$ and $300 \mathrm{~K}$.

- Provided experimental evidence of inverse hysteresis in Nickel contacted short channel SWNT at 77K.

\section{UNDERGRADUATE INTERNSHIP}

Engineers India Ltd., Delhi, India (June 2001 - July 2001)

Continental Devices India Ltd., Delhi, India (June 2000 - July 2000)

- Trained on the multitasking tool (3in1- testing, labeling and packaging) for manufacturing Surface Mount Devices (Transistors and Diodes). 


\section{PEER REVIEWED JOURNAL PAPERS}

- T. Bansal, A. D. Mohite, H. Shah, C. Galande, A. Srivastava, J. B. Jasinski, P. M. Ajayan, B. W. Alphenaar, "Measurement of the electronic density of states in oxidized graphene" submitted to ACS Nano, 2010.

- H. Shah, A. D. Mohite, T. Bansal, B. W. Alphenaar, "Photovoltage Bleaching in Bulk Heterojunction Solar Cells through Population of the PCBM Singlet Exciton" Appl. Phys. Lett. 97, 263301, 2010.

- A. N. Sidrov, T. Bansal, P. J. Ouseph, G. U. Sumanasekera, "Graphene nanoribbons exfoliated from graphite surface dislocation bands by electrostatic force" Nanotechnology 21, 195704, 2010.

- B. Nagabhirava, T. Bansal, G. U. Sumanasekera, L. Liu, B. W. Alphenaar, "Gated spin transport through individual single-walled nanotube" Appl. Phys. Lett. 88, 023503, 2006.

- T. Bansal, B. Nagabhirava, A. D. Mohite, P. Gopinath, G. U. Sumanasekera, B. W. Alphenaar, "Fabrication of short-channel individual single-walled carbon nanotube devices for spin transport measurements" Proceedings of the IEEE-Nano, 2006.

- A. D. Mohite, B. Nagabhirava, T. Bansal, P. Gopinath, H. Shah, G. U. Sumanasekera, B. W. Alphenaar, "Resolving excitonic and free carrier transitions in single-wall carbon nanotubes using field enhanced photocurrent spectroscopy" Proceedings of the IEEE-Nano, 2006. 


\section{CONFERENCE PRESENTATIONS AND ABSTRACTS}

- T. Bansal, A. D. Mohite, C. Glande, A. Srivastava, H.Shah, P. K. Ajayan \& B. W. Alphenaar "Measurement of the electronic density of states versus oxygen coverage in oxidized graphene" presented at the American Physical Society March meeting, Portland, Oregon 2010.

- H. Shah, A. D. Mohite, T. Bansal \& B. W. Alphenaar "Resolving the charge transfer complex (CTC) state in organic heterojunction solar cell using capacitive photocurrent technique" presented at the American Physical Society March meeting, Portland, Oregon 2010.

- Buddika K. Abeyweera, H. Shah, T. Bansal, K. Medepalli \& B. W. Alphenaar "Fabrication techniques for high efficient organic polymer solar cell" presented at Engineers Day, University of Louisville, 2010.

- T. Bansal, A. D. Mohite, J. Jasinski, M. Sunkara \& B. W. Alphenaar "Band-gap tuning through progressive oxidation of graphene" presented at the American Physical Society March meeting, Pittsburgh, Pennsylvania 2009.

- T. Bansal, A. D. Mohite \& B. W. Alphenaar "Energy band gap tuning of graphene through progressive oxidation" presented at Engineers Day, University of Louisville, 2009.

- A. D. Mohite, H. Shah, T. Bansal \& B. W. Alphenaar "Enhancement mechanisms of exciton formation and dissociation in organic solar cells" presented at $14^{\text {th }}$ Annual Kentucky EPSCoR Conference, Louisville, KY October 2008. 
- A. D. Mohite, P. Gopinath. B. Nagabhirava, T. Bansal, A. Cholpadi \& B. W. Alphenaar "Nanotube based opto-electronic, Spintronic devices and sensors" presented at Engineers Day, University of Louisville, 2007.

- T. Bansal, B. Nagabhirava, A. Mohite, P. Gopinath \& B. W. Alphenaar "Evidence for Gated spin transport through individual semiconducting SWNTs" presented at the American Physical Society March meeting, Denver, Colorado 2007.

- A. D. Mohite, P. Gopinath, H. Shah, B. Nagabhirava, T. Bansal \& B. W. Alphenaar "Exciton Annihilation processes in individual SWNTs" presented at the American Physical Society March meeting, Denver, Colorado 2007.

- P.Gopinath, A. D. Mohite, H. Shah, B. Nagabhirava, T. Bansal \& B. W. Alphenaar "Influence of strain on the optical properties of single-wall nanotubes" presented at the American Physical Society March meeting, Denver, Colorado 2007.

- B. Nagabhirava, T. Bansal, G. Sumanasekera, L. Liu and B. Alphenaar. "Gated spin Transport through an Individual Single-Walled Nanotube" presented at the American Physical Society March meeting, Baltimore, MD, 2006.

- T. Bansal, B. Nagabhirava, P. Gopinath, A. D. Mohite and B. W. Alphenaar "Fabrication of short-channel individual single-walled carbon nanotube devices for spin transport measurements" presented at the IEEE Nanotechnology conference, Cincinnati, Ohio 2006.

- A. D. Mohite, P. Gopinath, B. Nagabhirava, T. Bansal, H. Shah, G. U. Sumanasekera and B. W. Alphenaar "Resolving excitonic and free carrier transitions in Single-wall carbon nanotubes using field enhanced photocurrent spectroscopy" presented at the IEEE Nanotechnology conference, Cincinnati, Ohio 2006. 


\section{ACHIEVEMENTS AND AWARDS}

- Invited Talk at the IEEE-Nano06, Cincinnati, OH. "Fabrication of short-channel individual single-walled carbon nanotube devices for spin transport measurements."

- Science Magazine, Editors' choice: Highlights of the recent literature, "Gated spin transport through individual single-walled nanotube," Science 2006, Vol. 311, 439.

- DDC Award, Sponsored by SIGS, University of Louisville, Jan 2011 - May 2011.

- Research Assistantship, May 2004 - Dec 2010. 